\author{
UNITED STATES \\ DEPARTMENT OF THE INTERIOR \\ GEOLOGICAL SURVEY \\ Water Resources Division
}

\title{
A COMPARATIVE STUDY OF MEAN-SECTION AND MID-SECTION METHODS FOR COMPUTATION OF DISCHARGE MEASUREMENTS
}

\author{
Prepared by \\ Kenneth B. Young
}

February 1950 


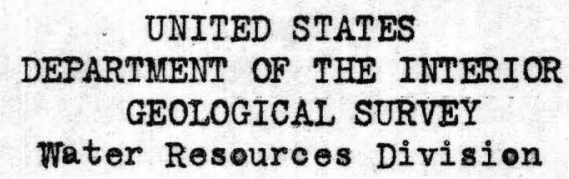

A COMParative STUDY OF

MEAN-SECTION AND MID-SECTION METHODS

FOR COMPUTATION OF

DISCHARGE MEASUREMENTS

Prepared by

Kenneth Bo Young

February 1950 

synopsis................................................

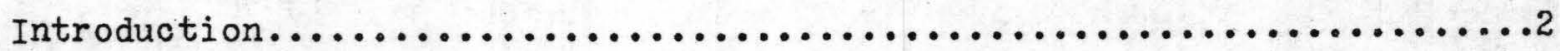

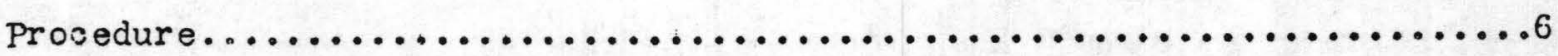

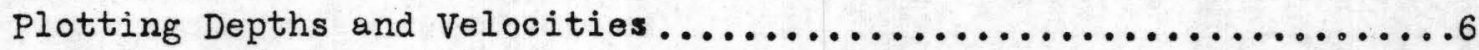

Selection of observation Stations ..........................

Computation of Normal Measurements..........................

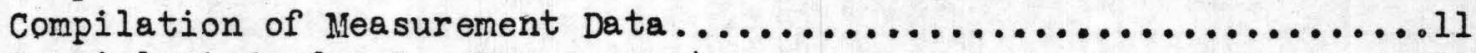

special study for Few Measurements ........................

Analysis of Relative Accuracy..............................

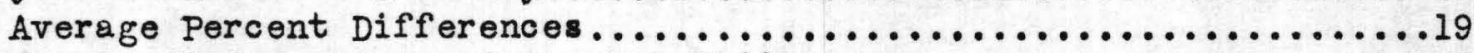

Graphical Comparison of Percent Differences...................

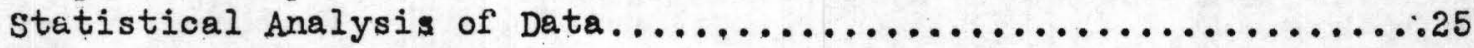

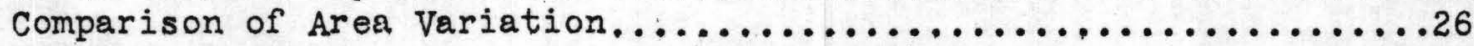

Effect of Velocity Component in Messurement................... 27

Study of Measurements with over Four Percent Difference.........28

Study of Three Measurements Satisfactory in original selections ...33

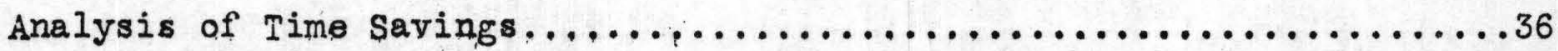

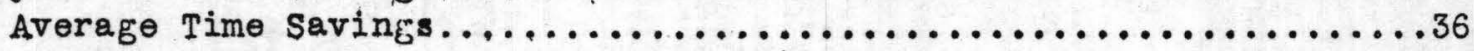

Graphical Comparison of Time Consumption.........................

Monetary Value of Time Savings.......................4

Discussion of Previous Investigations......................

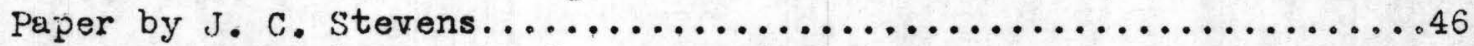

Use of Exact Formula for Comparison...................

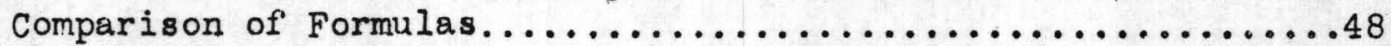

Discussion by C. E. Grunsky.........................49

Discussions in Water Resources Bulletin...................49

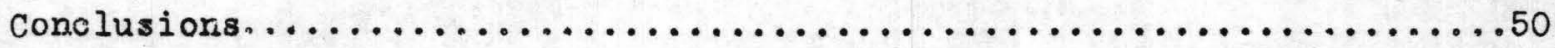





A COMPARATIVE STUDY OF
MEAN-SECTION AND MID-SECTION METHODS
FOR COMPUTATION OF
DISCHARGE MEASUREMENTS

SYNOPSIS

In February, 1949, Mr。 J。 V. 8. Wells, Chief of the Surface Water Branch, appointed a committee of three hydraulic engineers assigned to the Washington office to make a comparative study of the relative merits of the mean-section and mid-section methods of computing discharge measurements. This was done in order that there might be a basis for either adhering to the present "official" method, or adopting another method as the "official" one. This study is based on a collection of field data consisting of discharge measurements made with approximately four times the usual number of observations. There was a total of 213 of these special measurements made by all the district offices。

The two methods of computation were examined as to acouracy and time savings. This was done by selecting observation stations from the special measurements to arrive at a synthetio measurement with the usual number of observations. This. normal measurement was then oomputed by both the meansection and mid-section method of computation for a comparison 
of results in discharge. A record was kept of the time consumed for computing and checking measurements so that any time savings in either method could be found. Much of the computation work was done in four of the district offices, with a variety in personnel as to grade and to experience in the use of the two methods.

A review is made of previous investigations and discussions on the subject of methods of computing discharge measurements, particularly one by J. C. Stevens in 1908 which apparently was the basis of the adoption of the mean-section method as the "official" method. This portion of the study also serves as a sort of bibliography relating to papers on discharge computation methods.

The results of the study showed that, in general, the mid-section method is slightly more accurate than the mean-section method. Also, there is a considerable time savings in computing and checking measurements as a result of using the mid-section method.

\section{INTRODUCTION}

In the Water Resources Division of the Geological Survey, the method of computing current meter discharge measurements, in use since about 1908, is generally known as the "mean-section" method. This method is described in WSP 888, (pp 13-14) as follows: "In making a current-meter measurement, the total area of the cross section at the place of measurement is divided into small or partial sections and the area and the mean velocity of each is determined separately. The small sections are each bounded by the water surface, the stream bed, and two imaginary lines, called verticals. Fach vertical, therefore, being a common dimension 
for two ajoining sections, fixes the point at which observations are made. Suffioient velocity observations are made to establish the mean velocity in each of the two verticals forming the side boundaries of a section, and the velocities in the two verticals are then averaged to determine the mean velocity in the section. The product of the mean velocity thus obtained and the area of the section, which in turn is the product of the distance between the two verticals and the mean of their depths, is the discharge in the sections. The sum of the discharges in all the partial sections is the disoharge of the stream."

Another method of computation known as the "mid-section" method has bee come increasingly popular in recent years among some of the district offices of the Surface Water Branch. This method differs from the meansection" in that observations of depth and velocity are used directly without any averaging. Observations of depth and velocity are made in the same manner and the same points as in the mean-section method. The values of depth and velocity at each observation point apply to a cross-sectional area whose width extends half way to the preceding and following observation points. Consequently, in this computation, the process of averaging velocities to obtain a mean in section, and averaging depths to obtain a mean depth, are eliminated.

The midesection method of computation is not new. It was in use in the early years of the Geological Survey's water resources investigations but apparently was dropped after the adoption of the mean-section as the "standard method". At various times through the years since 1908 advocates of the mid-section method have urged its adoption as the "standard"。 The dvantages of one method over the other have been disoussed at length 
in the Water Resources Bulletin, (an official memorandum for use of Water Resources Division personnel) in a rather theoretical manner. There have been many statements made favoring the use of the mean-section method, and many favoring the adoption of the mid-section method as the "official" one. There was little question that a saving of time was possible using the mid-section method, but the matter of relative accuracy had not been definitely determined. Due to a lack of evidence to judge the merits of each method, and due to the advisability of having and using one official method of discharge measurement computation, it was decided to conduct a study to produce the necessary evidence which would show the facts.

In February, 1949, Mr. J。V.B.Wells, Chief of the Surface Water Branch, appointed a committee, consisting of Hollister Johnson, F. J. Flynn, and J. E. McCall, hydraulic engineers assigned to the Washington office, to make a comparative study of the mean-section and mid-section methods of computation. This committee decided a study based on actual field data was necessary for the formation of any conclusive opinions or decisions as to the merits of the methods. Accordingly, a plan of procedure was drawn up and a memorandum dated March 7; 1949, was sent to all district offices. This memorandum requested that each principal surface water field office furnish four current meter discharge measurements, each containing four times the usual number of sections and computed by both mean-section and mid-section methods. The committee felt that a measurement with four times the usual number of sections would be an acourate determination of the discharge, and that the computed disoharge of the abnormally long special measurements would be praotioally the same 
by both methods. The request for these special measurements specified that each type of current meter measurement be included; that is, cable, boat, bridge, and wading measurements. Also, the cross-section of three measurements should be typical of the region, and the fourth should have an irregular cross-section and velocity distribution. In this way, it was hoped to avoid any bias as to types of measurements and conditions, as well as to assure that results of the tests would be applicable to all types of measurements and all geographical locations.

The committee conducting this study realized that much extra work was entailed in making these special measurements and carrying the project through to a finish. There were 213 measurements submitted for this study, and the cooperation of the districts in complying with this. request is greatly appreciated. Four districts furnished the time and services of a considerable number of their personnel for two days in conducting computation time studies. The committee's appreciation and gratitude for this service go to the Boston, Charlottesville, College Park, and Columbus districts. Several engineers on detail in the Annual Reports Section of the Washington office assisted to a great extent in the computation time studies and their work is also much appreciated. Members of the Technical Coordination Branch gave suggestions in the statistical analysis of the data. The assignment of carrying out the work of compilation, computation, and analysis under the general supervision of the committee was given to K. B. Young, Hydraulic Engineer, in July 1949. 


\section{PROCEDURE}

\section{Plotting Depths and Velocities}

As the spectal measurements were received from the field offices, the depths and relocities were plotted to give a graphical picture of the crosssectior arid velocity distribution for each measurement. These plots were used later as a guide in selecting observation stations from each special measur ment for use in deriving a synthetic measurement, hereinafter called "normal measursment", having the customary number of observations, or sections。

\section{Selection of Observation Stations}

In selecting the observation stations for a normal measurement, consideration was given to the number of sections used in the past at the gaging station corserned, to the practice of keeping the partial discharge in each section undex 5 percent of the total discharge for the measurement, and to the orossmsection and velocity distribution as plotted. Observation stations ware selected as close as would be reasonable to banks and piers. An attempt was made to visualize what an engineer would do for each particular measurew ment in the matter of selecting the observation points. Where applicable, stations were selected to correspond to graduations, or multiples thereof, on the oable, bridge, or tagline. In other words, if 10 foot widths were satisfactory for defining the cross-section and flow, stations were picked at points $20,30,40$, etc., and not $22,32,42$, etc. The plotted depths and velooitjes served as a picture to furnish knowledge of river conditicns that an encineer actually measuring could see, or remember from previous 
measurements. In this way the section widths could be varied to get adequate definition around piers, boulders, banks, and other channel irregularities.

Previous to this study there seemed to be a general acceptance of a theory that to get an accurate discharge determination by the mid-section method, an engineer had to select observation stations with the thought in mind that the mid-section method of computation would be used. This theory, or belief, was that in making a discharge measurement for mid-section computation, it was necessary to avoid the mreaks" in cross-section and velocity. Accordingly, measurement 27 was treated in such a way that a combination of observation stations was selected for a mean-section com putation, and another combination was selected which was felt would give a bettex mid-section combination. This was the only measurement for which a different set of observation data was used for each method because it was felt that this theory is erroneous to a great extent and would lead to much extra work if observed. Therefore the practice of selecting points which give the best area definition should be used with due attention being given to velocity distribution.

When the observation stations for a normal measurement were selected, the basic field data including distances, depths, and mean velocities in the verticals were copied from the special measurement on regular discharge measurement note forms. Two identical sets of such data were made for each measurement, one for computation by the meansection method and the other, by the mid-section method. Special front sheets as shown in Fig. I were made up to attach to note forms so that 


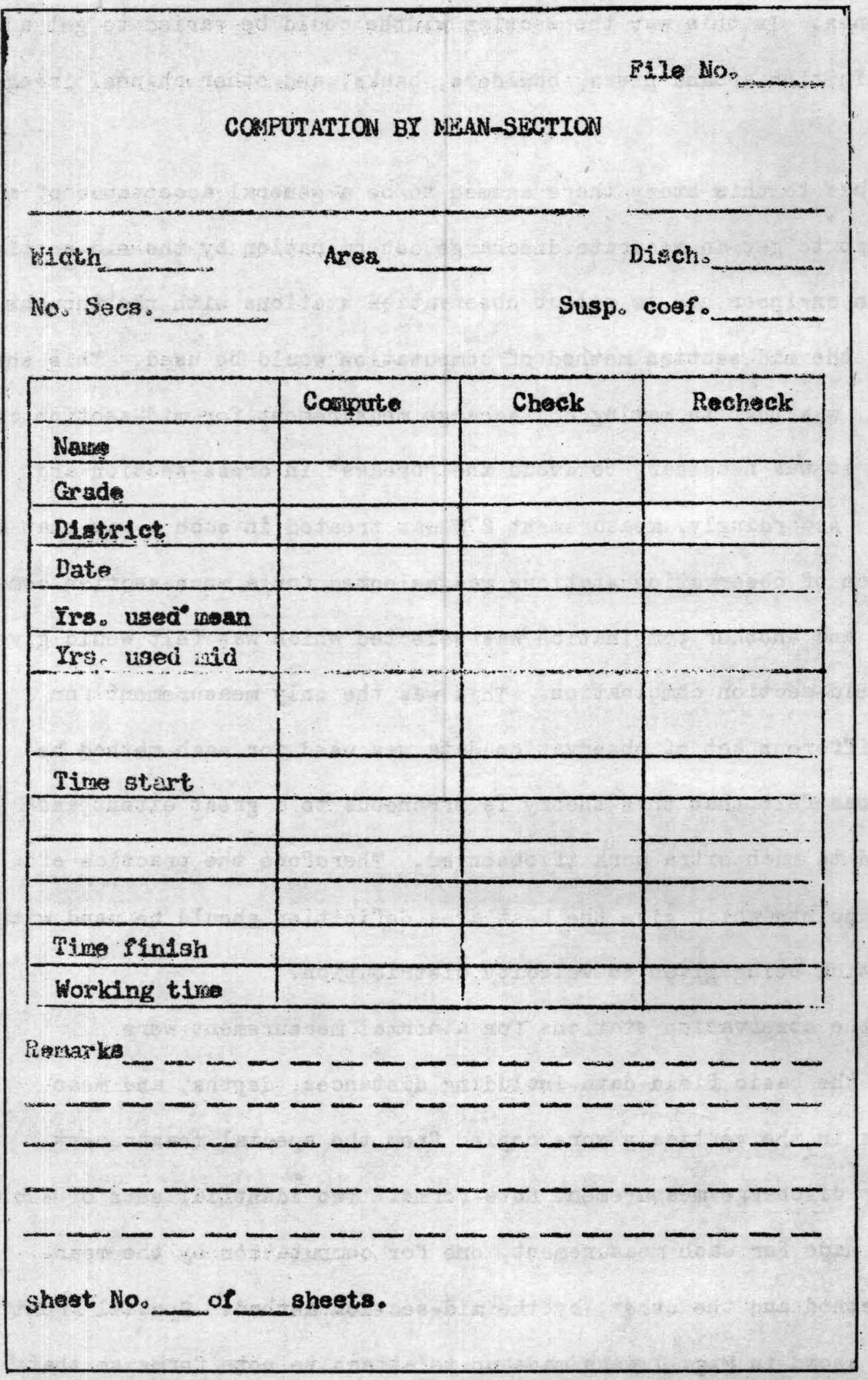

FIGURE 1.- Front sheet for use with normal measurement data. 
SAMPLE OF

DISCHARGE MEASUREMENT NOTES

COMPUTED BY MID-SECTION METHOD

\begin{tabular}{|c|c|c|c|c|c|c|c|c|c|c|c|c|}
\hline \multirow{2}{*}{ 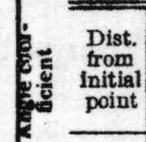 } & & & & & \multicolumn{3}{|c|}{ YRLOCITY } & \multirow[b]{2}{*}{ Area } & \multirow[b]{2}{*}{$\begin{array}{c}\text { Mean } \\
\text { depth }\end{array}$} & \multirow[b]{2}{*}{ width } & \multirow{2}{*}{\multicolumn{2}{|c|}{ Discharge }} \\
\hline & Dept & 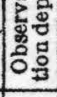 & $\begin{array}{c}\text { Rev- } \\
\text { olu- } \\
\text { tions } \\
\end{array}$ & $\begin{array}{l}\text { in } \\
\text { sec } \\
\text { onds } \\
\end{array}$ & point & $\begin{array}{c}\text { Mean } \\
\text { in vere- } \\
\text { tical }\end{array}$ & $\begin{array}{l}\text { Mean } \\
\text { in } \\
\text { inction }\end{array}$ & & & & & 8.92 \\
\hline \multicolumn{13}{|c|}{ L.E.W. at 9:05 A.M. } \\
\hline 0 & 0 & & 0 & & & 0 & & 0 & & 3 & 0 & .96 \\
\hline & & & & & & & & & & & & (8) \\
\hline \multirow[t]{2}{*}{6} & 2.6 & .6 & 7. & 52 & 0.30 & 0.30 & & 18.2 & & 7 & 5.5 & .98 \\
\hline & & & & & & & & & & & & \\
\hline \multirow[t]{2}{*}{14} & 5.9 & .2 & 20 & 54 & .81 & .68 & & 47.2 & & 8 & 32.1 & \\
\hline & & .8 & 15 & 60 & .55 & & & & & & & \\
\hline \multirow[t]{2}{*}{22} & 7.1 & .2 & 25 & 47 & 1.16 & .95 & & 49.7 & & 7 & 47.2 & \\
\hline & & .8 & 20 & 59 & .74 & & & & & & & \\
\hline \multirow[t]{2}{*}{28} & 8.2 & .2 & 30 & 52 & 1.26 & 1.11 & & 49.2 & & 6 & 54.6 & \\
\hline & & .8 & 25 & 57 & .96 & & & & & & & \\
\hline \multirow[t]{2}{*}{34} & 9.0 & .2 & 40 & 57 & 1.53 & 1.28 & & 54.0 & & 6 & 69.1 & \\
\hline & & .8 & 25 & 53 & 1.03 & & & & & & & \\
\hline \multirow[t]{2}{*}{40} & 9.2 & .2 & 40 & 54 & 1.61 & $1.4 \mathrm{~d}$ & & 50.6 & & 5.5 & 70.8 & \\
\hline & & .8 & 25 & 46 & 1,18 & & & & & & & \\
\hline \multirow[t]{2}{*}{45} & 9.3 & .2 & 40 & 52 & 1.67 & 1.52 & & 46.5 & & 5 & 70.7 & -9 \\
\hline & & .8 & 40 & 63 & 1.38 & & & & & & & \\
\hline \multirow[t]{2}{*}{50} & 9.4 & .2 & 40 & 48 & 1.81 & 1.64 & & 47.0 & & 5 & 77.1 & \\
\hline & & .8 & 40 & 59 & 1.47 & & & & & & & \\
\hline \multirow[t]{2}{*}{55} & 9.5 & .2 & 40 & 49 & 1.77 & 1.70 & & 47.5 & & 5 & 80.8 & \\
\hline & & .8 & 40 & 53 & 1.64 & & & & & & & \\
\hline \multirow[t]{2}{*}{60} & 9.7 & .2 & 40 & 44 & 1.97 & 1.78 & & 48.5 & & 5 & 86.3 & \\
\hline & & .8 & 30 & 41 & 1.59 & & & & & & & \\
\hline \multirow[t]{2}{*}{65} & 9.7 & .2 & 50 & 52 & 2.09 & 1.82 & & 48.5 & & 5 & 88.3 & \\
\hline & & .8 & 40 & 56 & 1.55 & & & & & & & \\
\hline \multirow[t]{2}{*}{70} & 10.0 & .2 & 40 & 43 & 2.02 & 1.82 & & 50.0 & & 5 & 91.0 & \\
\hline & & .8 & 40 & 54 & 1.61 & & & & & & & .96 \\
\hline \multirow[t]{2}{*}{7.5} & 10.3 & .2 & 40 & 44 & 1.97 & 1.72 & & 41.2 & & 4 & 70.9 & -97 \\
\hline & & .8 & 30 & 44 & 1.48 & & & & & & & .98 \\
\hline \multirow{2}{*}{$\frac{78}{\text { PIER }}$} & $\begin{array}{l}\text { est. } \\
10.0\end{array}$ & 8 & 0.9 & of 5 & a. 75 & 1.55 & & 15.0 & & 1.5 & 23.2 & \\
\hline & - & $10:$ & 04 & $\frac{m_{2}}{M_{2}}$ & & & & & & 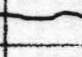 & & \\
\hline \multirow[t]{2}{*}{92} & $\begin{array}{r}\text { st. } \\
6.5 \\
\end{array}$ & & $\begin{array}{l}\text { est. } \\
0.9\end{array}$ & of st & a. 95 & .97 & & 9.8 & & 1.5 & 9.5 & \\
\hline & & & & & & & & & & & & \\
\hline \multirow[t]{2}{*}{95} & 6.8 & .2 & 30 & 51 & 1.28 & 1.08 & & 30.6 & & 4.5 & 33,0 & \\
\hline & & .8 & 20 & 50 & .87 & & & & & & & \\
\hline
\end{tabular}

FIGURE la.- Sample discharge measurement notes showing computation by mid-section method. 
information resulting from the measurement computation could be recorded systematioaliy. Fig. la shows a sample mid-section method computation.

\section{Computation of Normal Measurements}

After the basic field measurement data for the 213 pairs of normal measurements were assembled and ready for computation, the first tests of measurement computation were made using personnel in the washington office, espeoially engineers on detail in the Annual Reports Section. in this way, the proposed method of handling the computations was given a test to see if the procedure was clear and if a.ll information needed for analysis was being obtained. Later, computation sessions were held at the Boston, Charlottesville, College Park, and Columbus district offices using district personnel under the supervision of one of the committee members who we.s present to furnish instruction and information.

In choosing the districts to conduct the computation studies, the thought was to try to get personnel who were experienced in using the midesection as weli as the mean-section method, and to get a variety in the grades of personnel. It was difficult to get a very laxge peruentage of those experienced only in the mid-section method 8. most engineers who were using the mid-section method had changed over from the mean-section method and had thus become experienced in both methods. A majority of those used in the computations were engineers experienced in mean-section method, having had little contact with the mid-section method. Due to this fact, the average 
time aving shown by this study is probably somewhat lower than the saving that would be posaible if all personnel were equally familiar with the mid-section method。

The time (measured with stop watches) recorded for computation included only the processes from averaging the velocities (in, the case of the meansection method) to obtaining the partial discharges。 Other operations such as determining the mean velocity in the verticals, adding the partial areas and discharges, and filling out the front sheet which would be identical in both methods were not included in the time recorded. To have based this time study on the computation of an entire measurement, beginning with the application of velocities from the meter rating table, would have doubled the time required to carry out the procedure of getting the measurement computed. Therefore, the times measured are not the times needed to compute measurements, but give an indication of the difference in time between the two methods. Each person computed a measurement by both the mean-section and mid-section method. Later another person checked this "set"。 Thus assuming that the computer or checker worked at the same pace for erch method, the difference in time between methods was clearly indicated. After the messurements were computed and checked, they were examined for any remaining inconsistencies.

Compilation of Measurement Data

The measurements were separated into three groups of (1) Bridge, (2) Wading, and (3) Cable or Boat. They were numbered in their respeotive groups with the letter $W, B$, or $C$ added to indicate the type of 
measurement. The measurements of each group were listed in descending order of magnitude of the discharge as found in the special measurements (see rable 1). The necessary data were recorded in the proper columns, which are self-explanatory. Since there is a differenoe in the number of sections in the mean-section and mid-section computations, it was decided to use a column heading of "number of observations" instead of the usual "number of sections". In this way, the figure would be the same for both methods. When identioal observation points are used, the midsection method has one more section, or partial discherge, than the meansection method for each channel measured. In the case of most wading and cable measurements this difference is only one, but in bridge measurements the difference depends upon the number of channels.

The remaining columns were filled in by computation of the necessary items. The percent difference figure for discharge under each normal measurement is the percent difference from the true discharge which has been assumed to be the average of the mean-section and mid-section computation of the special measurement. The percent difference column for area is the differenoe of the normal-measurement area from the special-measurement area. The difference column under computation and checking time shows the saving of time in minutes by the mid-section method compared to the mean-section method. A negative figure indicates that the mid-section method took longer to compute or check than the meanosection method. A symbol was placed with the grade designation of the computers in order to classify them into three groups. 
BRIDGE MEASUREMENTS

\begin{tabular}{|c|c|c|c|c|c|c|c|c|c|c|c|c|c|c|c|c|c|c|c|c|c|c|}
\hline \multicolumn{2}{|c|}{ Gaging Station } & \multirow[b]{3}{*}{$\begin{array}{c}\text { File } \\
\text { No. }\end{array}$} & \multirow[b]{3}{*}{$\begin{array}{l}\text { No. } \\
\text { Obs. }\end{array}$} & \multicolumn{4}{|c|}{ Special Measurement } & \multicolumn{7}{|c|}{ Normal Measurement } & \multicolumn{4}{|c|}{ Computation } & \multicolumn{4}{|c|}{ Checking } \\
\hline \multirow[b]{2}{*}{ River } & \multirow[b]{2}{*}{ Place } & & & \multirow[b]{2}{*}{ Area } & \multirow[b]{2}{*}{$\begin{array}{l}\text { Mean- } \\
\text { Sec. }\end{array}$} & \multirow[b]{2}{*}{$\begin{array}{l}\text { Mid- } \\
\text { Sec. }\end{array}$} & \multirow[b]{2}{*}{ Average } & \multirow[b]{2}{*}{$\begin{array}{l}\text { Mean- } \\
\text { Sec. }\end{array}$} & & & & & & & Time & $\mathrm{e}$ in $\mathrm{Min}$ & autes & & Time & in Mifin & futes & \\
\hline & & & & & & & & & $\begin{array}{l}\text { cent } \\
\text { Diff. }\end{array}$ & $\begin{array}{l}\text { Mid- } \\
\text { Sec. }\end{array}$ & $\begin{array}{l}\text { cent } \\
\text { Diff. }\end{array}$ & Area & \begin{tabular}{|l|} 
cent \\
Diff. \\
\end{tabular} & \begin{tabular}{|l|} 
No. \\
Obs. \\
\end{tabular} & $\begin{array}{c}\text { Mean- } \\
\text { Sec. }\end{array}$ & Diff. & $\begin{array}{c}\text { Mid- } \\
\text { Sec. }\end{array}$ & rade & $\begin{array}{c}\text { Mean- } \\
\text { Sec. }\end{array}$ & Diff. & $\begin{array}{l}\text { Mid- } \\
\text { Sec. }\end{array}$ & Grade \\
\hline Missi & St. Louis, Mo. & $1 \mathrm{~B}$ & 172 & 43,470 & 152,175 & 152,241 & 152, & 151,876 & -0.2 & & +0.2 & 4 & -0.2 & 51 & 6.0 & 32.0 & 34.0 & $\mid \dagger \mathrm{SP}-5$ & 26.8 & 7.0 & 19.8 & $+P-2$ \\
\hline Missi & окик, & $2 \mathrm{~B}$ & 95 & 27,450 & 106,721 & 105,847 & 106,284 & 03,843 & $\mid-2.3$ & 103,748 & -2.4 & & $-\mathrm{C.} .9$ & 67 & 8 & 4.9 & 58.9 & & 57.5 & 13.5 & 44.0 & \\
\hline Arkansas & Van Buren, Ark. & 3B & 168 & 22,146 & 72,495 & 72,685 & 72,590 & 69,650 & -4.0 & 70,680 & -2.6 & 31,864 & -1.3 & 54 & 79.1 & 38.6 & 40.5 & * P-2 & 23.1 & 2,0 & 21.1 & -7 \\
\hline Missouri & Boonville, Mo. & $4 \mathrm{~B}$ & 145 & 15,247 & 54,283 & 54,387 & 54,335 & 53,744 & -1.1 & 54,374 & +0.1 & 15,040 & -1.4 & 49 & 40.1 & 12.3 & 27.8 & $\dagger \mathrm{HFA}$ & 39.3 & 9,1 & 30.2 & $P-4$ \\
\hline Red & Shreveport, La. & $5 B$ & 166 & 13,182 & 46,694 & 46,570 & 46,632 & 45,780 & -1.8 & 46,000 & -1.4 & 13,180 & & 43 & 32.0 & 11.0 & 21.0 & * P $\quad$-4 & 19.8. & 4.3 & 15.5 & $\mathrm{P}-3$ \\
\hline Tennessee & Chattanooga, Tenr & $6 \mathrm{~B}$ & 175 & 18,241 & 33,268 & 33,335 & 33,302 & 33,233 & -0.2 & 33,331 & +0.1 & 18,191 & -0.3 & 53 & 31.8 & 6.3 & 25.5 & * SP-4 & 31.0 & 4.8 & 26.2 & *State \\
\hline Kansas & Topeka, Kans. & $7 \mathrm{~B}$ & 105 & 6,318 & 32,761 & \begin{tabular}{|l|}
32,803 \\
\end{tabular} & 32,782 & 31,974 & -2.5 & 32,275 & -1.7 & 6,233 & -1.3 & 39 & 27.0 & 5.0 & 22.0 & $\dagger \mathrm{HFA}$ & 24.8 & 10.0 & 14.8 & * P-3 \\
\hline Potomac & Point of Rocks, Md. & $63 \mathrm{~B}$ & 207 & 8,213 & 28,959 & 28,920 & 28,940 & 28,078 & -3.0 & 28,283 & -2.3 & 8,142 & -0.9 & 65 & 52.0 & 6.0 & 46.0 & $* \begin{array}{ll}* \\
* \dagger\end{array}$ & 26.2 & 6.2 & 22.0 & * P-4 \\
\hline Missouri & Pierre, S. D. & $8 \mathrm{~B}$ & 118 & 9,723 & 28,268 & 28,346 & 28,307 & 28,300 & & 28,229 & $-0.3 \mid$ & 9,950 & +2.3 & 38 & 49,8 & 14.8 & 35.0 & * SP-5 & 26.0 & $\begin{array}{r}0.8 \\
-2.5\end{array}$ & 28.5 & * $\quad \mathrm{P}-2$ \\
\hline Alabama & Selma, Ala. & $9 \mathrm{~B}$ & 106 & 9,810 & 27,324 & 27,307 & 27,316 & 26,903 & -1.5 & 27,016 & $|-1.1|$ & 9,772 & $-0.4 \mid$ & 35 & 24.8 & 5.5 & 19.3 & & 17.9 & 4.0 & 13.9 & $* \dagger P-3$ \\
\hline Allegheny & Kittar & $10 \mathrm{~B}$ & 120 & 10,372 & 25,036 & 25,076 & 25,056 & 25,127 & +0.3 & 25,294 & +1.0 & 10,390 & +0.2 & 39 & 20.7 & $-0.4 \mid$ & 21.1 & & 17.4 & 0.2 & 17.2 & * $\quad \mathrm{P}-4$ \\
\hline Missou & N.D. & $11 \mathrm{~B}$ & 191 & 9,989 & 24,349 & 24,349 & 24,349 & 24,800 & +1.9 & 24,716 & +1.5 & 10,042 & +0.5 & 50 & 36.5 & 10.5 & 26.0 & * $\quad \mathrm{P}-3$ & 28.4 & 8.2 & 20.2 & * P-2 \\
\hline Arkan & Okla. & $12 \mathrm{~B}$ & 85 & 8,358 & 22,228 & 22,285 & 22,256 & 22,457 & $\begin{array}{r}+.9 \\
+0.9\end{array}$ & 22,580 & +1.4 & 8,366 & +0.1 & 52 & 33.2 & 3.2 & 30.0 & * $\quad \mathrm{P}-\mathbf{3}$ & 20.7 . & 5.5 & 15.2 & *† P-3 \\
\hline Yelío & Mont. & $13 \mathrm{~B}$ & 103 & 3,752 & 20 , & 20,750 & 6 & 20,697 & -0.1 & 20,971 & $\begin{array}{r}+1.7 \\
+1.2\end{array}$ & 3,728 & -0.6 & 29 & 21.4 & 6.0 & 15.4 & $* \quad \mathbf{P}-2$ & 15.5 & 4.0 & 11.5 & * $\mathrm{P}-1$ \\
\hline Sabine & Tav & $14 \mathrm{~B}$ & 102 & 7,360 & 19,112 & 19,146 & 9 & 19,204 & +0.4 & 3 & +0.7 & 8 & +1.2 & $35>2>$ & 38.5 & 3.0 & 35.5 & $\dagger \quad \mathrm{P}-2$ & 21.5 & 3.3 & 18.2 & $\dagger \mathrm{SP}-5$ \\
\hline Skagit & Mt. Vernon, Wash & $15 \mathrm{~B}$ & 120 & 4,972 & 15,478 & 15,488 & 33 & 14,848 & -4.1 & 15,031 & -2.9 & 4,894 & -1.6 & 46 & 5 & 14.6 & 30.7 & * $\quad$ P-3 & & $\begin{array}{r}0.0 \\
-4.5\end{array}$ & $\mid$ & $* \quad \mathrm{P}-2$ \\
\hline Allegheny & Franklin, Pa. & $16 \mathrm{~B}$ & 123 & 4,732 & 15,153 & 15,221 & 15,187 & 15,263 & $\begin{array}{r}+0.5 \\
\end{array}$ & 15,339 & +1.0 & 4,782 & +1.1 & 42 & 22.0 & 4.8 & 17.2 & * $\quad$ P-4 & 2 & -0.4 & 18.6 & $* \quad P-4$ \\
\hline Des Moines & owa & $17 \mathrm{~B}$ & 65 & 4,135 & 14,932 & 15,032 & 14,982 & 14,103 & -5.9 & 14,313 & -4.5 & 4,032 & -2.5 & 33 & 22.3 & 8.1 & 14.2 & * P-3 & 18,8 & 5.5 & 13.3 & * P-1 \\
\hline D & J. & $18 \mathrm{~B}$ & 316 & 5,525 & 12,070 & 12,080 & 12,075 & 12,11 a & +0.3 & 12,125 & $\begin{array}{r}-.0 \\
+0.4\end{array}$ & 5,536 & +0.2 & 91 & 61.5 & 9.0 & 52.5 & $\dagger \quad \mathrm{P}-2$ & 59.0 & 11.5 & 47.5 & t SP-3 \\
\hline Delaware. & N. Y & $19 \mathrm{~B}$ & 127 & 3,524 & 11,824 & 11,832 & 8 & 01 & $\begin{array}{l}0.0 \\
-0.3\end{array}$ & 32 & 0 & 3,554 & +0.9 & 34 & 20.9 & 5.8 & 15.1 & $\begin{array}{ll}* & \mathbf{P}-3\end{array}$ & 11.6 & -0.1 & 11.7 & * P-4 \\
\hline Susquehanna & tal. & $20 \mathrm{~B}$ & 109 & 4,832 & 9,722 & 9,724 & 9,723 & 9,715 & -0.1 & 57. & +0.3 & 4 & -0.8 & (32 & 16.8 & -2.1 & 18.9 & * $\quad \mathrm{P}-3$ & 14.4 & -0.8 & 15.2 & * $\quad \mathrm{P}-3$ \\
\hline Wabash & Ind. & $21 \mathrm{~B}$ & 104 & 5,785 & 7,770 & 7,790 & 7,780 & 7,563 & -2.8 & 7,687 & -1.2 & 5,768 & -0.3 & 4 & $\begin{array}{l}.0 \\
5.7\end{array}$ & 11.5 & $\begin{array}{l}1.9 \\
24.2\end{array}$ & * $\quad \mathrm{P}-1$ & & 6.0 & \begin{tabular}{|l|}
18.0 \\
18.0
\end{tabular} & * $\mathrm{P}-2$ \\
\hline James & 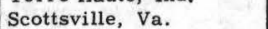 & $22 \mathrm{~B}$ & 109 & 3,482 & 7,544 & 7,557 & 7,550 & 7,302 & -3.3 & 7,296 & -3.4 & 3,452 & -0.9 & 37 & 23.6 & 0.9 & 22,7 & $\left|\begin{array}{ll}* & 0-1 \\
* & \mathrm{P}-2\end{array}\right|$ & 0 & $\begin{array}{l}.00 \\
4.3\end{array}$ & $\begin{array}{l}18.0 \\
17.7\end{array}$ & * $\quad \mathrm{P}-2$ \\
\hline Chippewa & D & $23 \mathrm{~B}$ & 153 & 2,813 & 6,942 & 6,941 & 6,942 & 6,933 & -0.1 & 6,985 & +0.6 & 2,780 & -1.2 & 50 & 37.3 & 6.4 & 30.9 & $\begin{array}{ll}\dagger & \mathrm{P}-2\end{array}$ & 7.0 & 15.7 & 21.3 & $\dagger \quad \mathrm{P}-2$ \\
\hline & 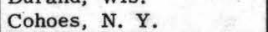 & $24 \mathrm{~B}$ & 122 & 5,226 & 6,548 & 6,543 & 6,546 & 6,588 & $\begin{array}{r}0.6 \\
+0.6\end{array}$ & 6,608 & +1.0 & 5,178 & -0.9 & 46 & 18.5 & -3.0 & 21.5 & * $\quad P-3$ & 8.7 & -0.5 & 19.2 & P-3 \\
\hline & M & | & 97 & & & 6,359 & 6,3 & $\begin{array}{l}0,300 \\
6,379\end{array}$ & $\begin{array}{l}+0.0 \\
+0.4\end{array}$ & 6, 434 & $\begin{array}{r}1.0 \\
+1.2 \\
\end{array}$ & 3,488 & +1.0 & 33 & 29.9 & 9.4 & 20.5 & * $\quad \mathrm{P}-3$ & 12.2 & 3.2 & 9.0 & * SP-7 \\
\hline $\begin{array}{l}\text { Lear } \\
\text { Pee Dee }\end{array}$ & s. & $26 \mathrm{~B}$ & $\begin{array}{r}304 \\
\end{array}$ & $\begin{array}{l}3,450 \\
3,706\end{array}$ & 6,2 & 6,254 & 6,2 & 88 & $\begin{array}{r}70 . t^{4} \\
+0.5\end{array}$ & 6,240 & -0.2 & 3,716 & +0.3 & 33 & 22 & 8.8 & 13.2 & $* \begin{array}{l}* \\
\mathrm{P}-3\end{array}$ & 13.7 & 2.9 & 10,8 & $* \mathrm{P}-3$ \\
\hline Barren & , Ky & $27 \bar{B}$ & 121 & 1,955 & 5, 539 & 5,543 & 5,541 & 5,801 & $-2,7$ & 5,437 & -1.9 & 1,968 & +0.7 & 3 & 14.0 & $\begin{array}{l}0.0 \\
2.4\end{array}$ & 11.6 & $* \quad \mathrm{P}-3$ & 2 & 3.2 & $\begin{array}{r}9.0 \\
9.0\end{array}$ & * P-3 \\
\hline iee & & $28 \mathrm{~B}$ & 150 & 1,618 & 4, 942 & 4,938 & 4,940 & 4,899 & -0.8 & 4,937 & -0.1 & 1,620 & +0.1 & 3 & $\begin{array}{l}33.8 \\
33.8\end{array}$ & 9.6 & 24.2 & * SP -5 & 2.1 & $\begin{array}{l}3.2 \\
1,2\end{array}$ & 20.9 & * P-2 \\
\hline Ro & e. & $29 \mathrm{~B}$ & 110 & 1 , & 3,930 & 3,928 & 3,934 & 3,855 & $-2,0 \mid$ & 3,892 & -1.1 & 1,372 & -1 & 3 & & 8.0 & 25.8 & $* P-2$ & 13.4 & 2.4 & 11.0 & $* \mathrm{SP}-7$ \\
\hline M & Elk R & зов & | & 3 & 3,877 & 3,873 & 3,875 & 3,812 & -1.6 & 3,855 & -0.5 & 2,428 & -0.2 & 3 & 19.9 & 3.4 & 16.5 & W P-2 & 17.2 & 1.2 & 16.0 & $\dagger \mathrm{HFA}$ \\
\hline & & $31 \mathrm{~B}$ & 83 & 3, & 3,613 & 3,614 & 3,614 & 3,726 & $\begin{array}{r}+3.1 \\
\end{array}$ & $\mathbf{3}, \mathbf{7 3 9}$ & $\begin{array}{r}+.5 \\
+3.5\end{array}$ & 3,833 & $-1.0 \mid$ & 31 & 21.8 & 4.1 & 17.7 & *† $\mathrm{P}-3$ & 15.2 & 0.7 & 14.5 & * SP-4 \\
\hline Junia & & $32 \mathrm{~B}$ & $\begin{array}{r}00 \\
116\end{array}$ & 2,211 & $\mid \begin{array}{l}3,010 \\
3,280\end{array}$ & $\left|\begin{array}{l}3,014 \\
3,276\end{array}\right|$ & 3,2 & 7 & $\begin{array}{l}+0.1 \\
-0.9\end{array} \mid$ & 3,314 & +1.1 & 2,137 & -3.4 & 3 & $\begin{array}{l}13.6 \\
\end{array}$ & 0.1 & 13.5 & $* \quad P-4$ & 10.9 & -0.7 & 11.6 & * P -4 \\
\hline $\mathrm{Yz}$ & ege, N. C. & 33B & 114 & 1,289 & 3,185 & 3,189 & 3,187 & 3,199 & +0.4 & 3,230 & $\begin{array}{r}+1.4 \\
+1.4\end{array}$ & 1,280 & -0.7 & & 6.0 & 11.1 & 14.9 & * $\quad$ P-2 & 10.0 & 0.6 & 9.4 & $* \mathrm{SP}-7$ \\
\hline non & te $>$ & $34 \mathrm{~B}$ & 106 & 1,4 & 3,1 & 3,116 & 3,111 & 3,102 & -0.3 & 3,123 & +0.4 & 1,436 & -0.8 & 3 & 19.6 & $\begin{array}{l}6.2 \\
6.2\end{array}$ & 13.4 & * $\quad \mathrm{P}-2$ & 14.7 & 0.5 & \begin{tabular}{|r|}
0.7 \\
14.2
\end{tabular} & * $\mathbf{P}-1$ \\
\hline Tuala & Willar & $35 \mathrm{~B}$ & 82 & 8 & 2,993 & 2,991 & 2,992 & 3,052 & +2.0 & 3,074 & +2.7 & 3,210 & +0.1 & 2 & 24.8 & 7.8 & 17.0 & * P-2 & 8.6 & 2.4 & 6.4 & * $\mathrm{SP}-7$ \\
\hline Fre & & $36 \mathrm{~B}$ & $\begin{array}{r}02 \\
131\end{array}$ & 1,553 & 2,886 & 2,892 & 2,889 & 2,896 & +0.2 & 2,883 & -0.2 & 1,559 & +0.4 & 42 & 22.4 & 4.7 & 17.7 & $* \mathrm{SP}-4$ & 19.8 & 1.9 & 17.9 & *State \\
\hline Grand-River & Mich. & $37 \mathrm{~B}$ & \begin{tabular}{|l}
101 \\
120
\end{tabular} & 1,752 & 2,808 & 2,805 & 2,8 & 2,763 & -1.5 & 2,765 & -1.5 & 1,717 & $\left|\begin{array}{l}0.7 \\
-2.0\end{array}\right|$ & 3 & 18.2 & $\begin{array}{l}4.7 \\
4.7\end{array}$ & 13.5 & * P-2 & 14.5 & 2.0 & 12.5 & * SP-5 \\
\hline Arkan & Kans. & $38 \mathrm{~B}$ & 126 & 1,115 & 2,742 & & 2,7 & 2,546 & $\begin{array}{l}-1.0 \\
-7.4\end{array}$ & 2,594 & $|-5.7|$ & 1,100 . & -1 & & & 7.0 & & & & 10.0 & 13.8 & $* \dagger^{2} \mathrm{P}-2$ \\
\hline & & & 12 & & & & 2,4 & 2,491 & $\begin{array}{r}1.4 \\
+1\end{array}$ & 2,506 & $\begin{array}{r}0.8 \\
+1.8\end{array}$ & 984 & {$\left[\begin{array}{l}1.4 \\
+0.4\end{array}\right]$} & 4 & 5 & 13.0 & & {$\left[\begin{array}{ll}1 & \\
+ & \text { p-1 }\end{array}\right]$} & & 9.4 & 20.4 & $\dagger \quad P-2$ \\
\hline ande & $\mathrm{S} z$ & 62 & 129 & 67 & 2,2 & 2,2 & & 2,262 & -1.0 & 2,275 & -0.4 & . & $\begin{array}{l}+0.4 \\
+0.3\end{array} \mid$ & 3. & 24.8 & $\begin{array}{r}4.0 \\
4.6\end{array}$ & 20.2 & $* \dagger$ P- 4 & $\begin{array}{l}29.6 \\
13.2\end{array}$ & $\begin{array}{l}8.4 \\
2.4\end{array}$ & $\begin{array}{l}80.4 \\
10.8\end{array}$ & $*{ }_{1}+\mathrm{P}-4$ \\
\hline Licking & prings, $\mathrm{Ky}$. & $40 \mathrm{~B}$ & 115 & 1,124 & 2,269 & 2,276 & 2,272 & 2,250 & -1.0 & 2,277 & +0.2 & 1,114 & -0.9 & 33 & 14.5 & 4.8 & 9.7 & {$\left[\begin{array}{ll}* & \mathbf{p}-3\end{array}\right]$} & 14.0 & $\begin{array}{l}8.4 \\
4.6\end{array}$ & 9.4 & * P-8 \\
\hline Senac & Anders & $41 \mathrm{~B}$ & 105 & 1,370 & 2,143 & 2,143 & 2,143 & 2,103 & -1.9 & 2,107 & $|-1.7|$ & 1,361 & $-0.7 \mid$ & 36 & 19.6 & 4.4 & 15.2 & $* \dagger \mathrm{P}-3$ & 16.5 & 3.4 & 13.1 & * $P-4$ \\
\hline & Enter & $42 B$ & $\begin{array}{r}81 \\
81\end{array}$ & 599 & 2,002 & $\begin{array}{l}2,005 \\
\end{array}$ & 2,004 & 1,975 & -1.4 & 2,004 & 0 & 596 & -0.5 & 27 & 22.2 & 3.0 & 19.2 & * $\quad \mathrm{P}-3$ & 6.7 & 1.0 & 5.7 & * P-2 \\
\hline $\begin{array}{l}\text { sipee } \\
\text { sipe }\end{array}$ & Effingham Falls, N. н. & $43 \mathrm{~B}$ & 100 & 1,768 & 1,881 & 1,886 & 1,884 & 1,847 . & -2.0 & 1,856 & -1.5 & 1,752 & $|-0.9|$ & 30 & 17.6 & 6.0 & 11.6 & *† P-4 & 17.5 & 4.7 & 12.8 & $\dagger \mathbf{p}-2$ \\
\hline
\end{tabular}

\section{* Experienced in mean-section method}

*† Experienced in both methods 
TABLE 1 - Continued

\begin{tabular}{|c|c|c|c|c|c|c|c|c|c|c|c|c|c|c|c|c|c|c|c|c|c|c|}
\hline \multicolumn{2}{|c|}{ Gaging Station } & \multicolumn{6}{|c|}{ Special Measurement } & \multicolumn{7}{|c|}{ Normal Measurement } & \multicolumn{4}{|c|}{ Computation } & \multicolumn{4}{|c|}{ Checking } \\
\hline River & Place & $\begin{array}{c}\text { File } \\
\text { No. }\end{array}$ & $\begin{array}{l}\text { No. } \\
\text { Obs. }\end{array}$ & Area & $\begin{array}{l}\text { Mean- } \\
\text { Sec. }\end{array}$ & $\begin{array}{l}\text { Mid- } \\
\text { Sec. }\end{array}$ & Average & $\begin{array}{l}\text { Mean- } \\
\text { Sec. }\end{array}$ & $\begin{array}{l}\text { Per- } \\
\text { cent } \\
\text { Diff. }\end{array}$ & $\begin{array}{l}\text { Mid- } \\
\text { Sec. }\end{array}$ & \begin{tabular}{|l|} 
Per- \\
cent \\
Diff.
\end{tabular} & Area & \begin{tabular}{|l|} 
Per- \\
cent \\
Diff. \\
\end{tabular} & $\begin{array}{l}\text { No. } \\
\text { Obs. }\end{array}$ & \begin{tabular}{|r|}
\multicolumn{2}{|c}{ Time } \\
Mean- \\
Sec. \\
\end{tabular} & \begin{tabular}{|l|} 
in Min \\
Diff.
\end{tabular} & \begin{tabular}{c|} 
utes \\
Mid- \\
Sec.
\end{tabular} & Grade 1 & \begin{tabular}{|c|} 
Time \\
Mean- \\
Sec.
\end{tabular} & \begin{tabular}{|l|} 
in Minu \\
Diff.
\end{tabular} & \begin{tabular}{|c|} 
utes \\
Mid- \\
Sec.
\end{tabular} & Grade \\
\hline $\begin{array}{l}\text { French Broad } \\
\text { Cheyenne } \ddagger \\
\text { Choctawhatchee } \\
\text { Tygart } \\
\text { Gila } \\
\text { St. Johns } \\
\text { Bighorn } \\
\text { Colorado } \\
\text { Kalamazoo } \\
\text { Red Cedar } \\
\text { Colorado } \\
\text { Chatooga } \\
\text { Taylor } \\
\text { Amite } \\
\text { Scioto } \\
\text { Taunton } \\
\text { Oklawaha } \\
\text { Colorado }\end{array}$ & $\begin{array}{l}\text { Asheville, N. C. } \\
\text { Eagle Butte, S. D. } \\
\text { Newton, Ala. } \\
\text { Colfax, W. Va. } \\
\text { Calva, Ariz. } \\
\text { Sanford, Fla. } \\
\text { Rairden, Wyo. } \\
\text { Wharton, Tex. } \\
\text { Comstock, Mich. } \\
\text { Menomonie, Wis. } \\
\text { LaGrange, Tex. } \\
\text { Clayton, Ga. } \\
\text { Almont, Colo. } \\
\text { Denham Springs, La. } \\
\text { Dublin, Ohio } \\
\text { State Farm, Mass. } \\
\text { Ocala, Fla. } \\
\text { Ballinger, Tex. }\end{array}$ & $\begin{array}{l}44 \mathrm{~B} \\
45 \mathrm{~B} \\
46 \mathrm{~B} \\
47 \mathrm{~B} \\
48 \mathrm{~B} \\
49 \mathrm{~B} \\
50 \mathrm{~B} \\
51 \mathrm{~B} \\
52 \mathrm{~B} \\
53 \mathrm{~B} \\
54 \mathrm{~B} \\
55 \mathrm{~B} \\
56 \mathrm{~B} \\
57 \mathrm{~B} \\
58 \mathrm{~B} \\
59 \mathrm{~B} \\
60 \mathrm{~B} \\
61 \mathrm{~B}\end{array}$ & \begin{tabular}{|r}
162 \\
126 \\
101 \\
123 \\
121 \\
120 \\
89 \\
86 \\
111 \\
105 \\
77 \\
148 \\
68 \\
82 \\
117 \\
104 \\
99 \\
61
\end{tabular} & $\begin{array}{r}740 \\
619 \\
441 \\
1,133 \\
528 \\
4,390 \\
402 \\
783 \\
706 \\
1,095 \\
732 \\
339 \\
188 \\
684 \\
815 \\
640 \\
429 \\
353\end{array}$ & $\begin{array}{r}1,730 \\
1,662 \\
1,560 \\
1,442 \\
1,383 \\
1,319 \\
1,309 \\
1,302 \\
1,129 \\
1,070 \\
909 \\
911 \\
819 \\
725 \\
525 \\
522 \\
341 \\
256\end{array}$ & \begin{tabular}{r|}
1,729 \\
1,665 \\
1,559 \\
1,443 \\
1,386 \\
1,318 \\
1,313 \\
1,304 \\
1,130 \\
1,070 \\
914 \\
909 \\
818 \\
725 \\
524 \\
522 \\
341 \\
256
\end{tabular} & $\begin{array}{l}1,730 \\
1,664 \\
1,560 \\
1,442 \\
1,384 \\
1,318 \\
1,311 \\
1,303 \\
1,130 \\
1,070 \\
912 \\
910 \\
818 \\
725 \\
524 \\
522 \\
341 \\
256\end{array}$ & $\begin{array}{r}1,718 \\
1,653 \\
1,558 \\
1,437 \\
1,368 \\
1,300 \\
1,312 \\
1,306 \\
1,141 \\
1,057 \\
873 \\
885 \\
819 \\
713 \\
517 \\
514 \\
345 \\
251\end{array}$ & $\begin{array}{l}-0.7 \\
-0.7 \\
-0.5 \\
-0.3 \\
-1.2 \\
-1.4 \\
+0.1 \\
+0.2 \\
+1.0 \\
-1.2 \\
-4.3 \\
-2.7 \\
+0.1 \\
-1.1 \\
-1.3 \\
-1.5 \\
+1.2 \\
-2.0\end{array}$ & $\begin{array}{r}1,735 \\
1,674 \\
1,556 \\
1,441 \\
1,382 \\
1,308 \\
1,312 \\
1,312 \\
1,125 \\
1,064 \\
885 \\
890 \\
826 \\
724 \\
\mathbf{5 2 0} \\
516 \\
349 \\
251\end{array}$ & $\begin{array}{l}+0.3 \\
+0.6 \\
-0.3 \\
0 \\
-0.1 \\
-0.8 \\
+0.1 \\
+0.7 \\
-0.4 \\
-0.6 \\
-3.0 \\
-2.2 \\
+1.0 \\
-0.1 \\
-0.8 \\
-1.1 \\
+2.3 \\
-2.0\end{array}$ & $\begin{array}{r}736 \\
614 \\
445 \\
1,124 \\
531 \\
4,384 \\
401 \\
785 \\
707 \\
1,100 \\
717 \\
329 \\
187 \\
673 \\
806 \\
634 \\
430 \\
351\end{array}$ & $\begin{array}{c}-0.5 \\
-0.8 \\
+0.9 \\
-0.8 \\
+0.6 \\
-0.1 \\
-0.2 \\
+0.3 \\
+0.1 \\
+0.5 \\
-2.0 \\
0 \\
-0.5 \\
-1.6 \\
-1.1 \\
-0.9 \\
+0.2 \\
-0.6\end{array}$ & $\begin{array}{l}43 \\
29 \\
32 \\
32 \\
42 \\
38 \\
29 \\
28 \\
30 \\
30 \\
28 \\
33 \\
27 \\
23 \\
33 \\
32 \\
27 \\
32\end{array}$ & \begin{tabular}{|r}
17.0 \\
14.6 \\
23.8 \\
12.7 \\
36.7 \\
29.3 \\
18.2 \\
14.5 \\
12.0 \\
16.8 \\
36.0 \\
14.6 \\
11.3 \\
9.0 \\
25.2 \\
20.0 \\
15.4 \\
20.4
\end{tabular} & \begin{tabular}{|r|}
3.8 \\
9.0 \\
8.9 \\
-5.5 \\
10.8 \\
5.4 \\
7.7 \\
3.4 \\
2.5 \\
4.7 \\
17.0 \\
5.3 \\
1.1 \\
1.5 \\
6.4 \\
3.0 \\
4.6 \\
5.4
\end{tabular} & $\begin{array}{r}13.2 \\
5.6 \\
14.9 \\
18.2 \\
25.9 \\
23.9 \\
10.5 \\
11.1 \\
9.5 \\
12.1 \\
19.0 \\
9.3 \\
10.2 \\
7.5 \\
18.8 \\
17.0 \\
10.8 \\
15.0\end{array}$ & 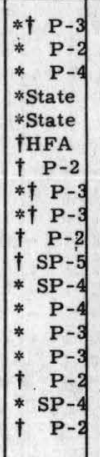 & $\begin{array}{r}15.5 \\
12.9 \\
15.6 \\
9.9 \\
16.0 \\
18.0 \\
17.1 \\
7.9 \\
16.8 \\
8.4 \\
10.3 \\
12.4 \\
11.0 \\
9.4 \\
9.6 \\
8.6 \\
10.6 \\
6.8\end{array}$ & \begin{tabular}{|r|}
3.7 \\
3.1 \\
6.5 \\
0.4 \\
1.5 \\
5.8 \\
5.6 \\
1.6 \\
10.5 \\
1.0 \\
2.2 \\
4.4 \\
3.0 \\
2.3 \\
3.8 \\
1.5 \\
1.0 \\
1.6
\end{tabular} & $\begin{array}{r}11.8 \\
9.8 \\
9.1 \\
9.5 \\
14.5 \\
12.2 \\
11.5 \\
6.9 \\
6.3 \\
7.4 \\
8.1 \\
8.0 \\
8.0 \\
7.1 \\
5.8 \\
7.1 \\
9.6 \\
5.2\end{array}$ & 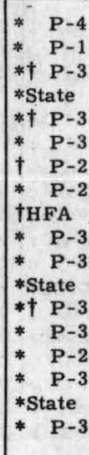 \\
\hline & & & & & & & & & & & & & & & & & & & & & & \\
\hline $\begin{array}{l}\text { Cedar } \\
\text { Kanka }\end{array}$ & laterioo & $1 \mathrm{w}$ & 64 & 993 & 2,314 & 2,315 & 3 & 2,302 & -0. & 2,306 & -0.3 & 993 & 0 & 28 & 21.6 & 6.3 & 15.3 & * P-2 & 3.0 & 2.7 & 10.3 & \\
\hline $\begin{array}{l}\text { Kankakee } \\
\text { Platte }\end{array}$ & & $2 W$ & 136 & 1,129 & 2,200 & 2,219 & 2,210 & 2,200 & -0.5. & 2,218 & +0.4 & 1,132 & +0.3 & & 30. & 16.0 & 14.5 & 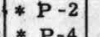 & 18.2 & 4.4 & 13.8 & \\
\hline $\begin{array}{l}\text { Platte } \\
\text { Tallapoosa }\end{array}$ & and, Neb. & $75 \mathrm{~W}$ & 232 & 839 & 1,607 & 1,615 & 1,611 & 1,647 & +2.21 & 1,662 & +3.2 & 853 & +1.7 & 62 & 28 & 2.8 & 25.3 & $\mid \begin{array}{l}* P-4 \\
*+P-3\end{array}$ & $\begin{array}{l}37.0 \\
20.0\end{array}$ & $\begin{array}{l}1.0 \\
8.7\end{array}$ & $\begin{array}{ll}36.0 \\
11\end{array}$ & *† P-3 \\
\hline Little Missouri & Ofel & $3 w$ & 110 & 476 & 1,153 & 1,155 & 1,154 & 1,177 & +2.0 & 1,178 & +2.1 & 477 & +0.2 & 29 & & & $\begin{array}{l}9.1 \\
8.6\end{array}$ & $\begin{array}{l}\text { *TP-3 } \\
\text { *TP-3 }\end{array}$ & & $\begin{array}{l}8.7 \\
1.0\end{array} \mid$ & $\begin{array}{l}11.3 \\
11.2\end{array}$ & \\
\hline $\begin{array}{l}\text { Chehalis } \\
\text {. }\end{array}$ & ound, & $4 \mathrm{~W}$ & 93 & 396 & 835 & 936 & 936 & 901 & -3.7 & 909. & -2.9 & 385 & +2.9 & 26 & $23+2+2$ & 4.5 & $\begin{array}{r}8.6 \\
16.7\end{array}$ & * P-3 & $\begin{array}{r}12.2 \\
7.2\end{array}$ & $\begin{array}{l}1.0 \\
1.2\end{array}$ & $\begin{array}{r}11.2 \\
6.0\end{array}$ & $t_{*}^{\dagger} \mathrm{SP}-5$ \\
\hline & $\begin{array}{l}\text { rand Mound, Wa } \\
\text { inkelman, Ariz. }\end{array}$ & $5 \mathrm{w}$ & 120 & 341 & 868 & 868 & 868 & 862 & -0.7 & 867 & -0.1 & 340 & -0.3 & 29 & $\begin{array}{l}23,0 \\
16,0\end{array}$ & 6. 6 ( 19 & 10.6 & *State & & 2.4 & $\begin{array}{l}7.2 \\
7.2\end{array}$ & $\begin{array}{ll}* & P-2 \\
* \dagger & P-3\end{array}$ \\
\hline Raystown Br. Juniata & $\begin{array}{l}\text { Winkelman, Ariz. } \\
\text { Saxton, Pa. }\end{array}$ & $\begin{array}{l}6 \mathrm{~W} \\
7 \mathrm{~W}\end{array}$ & $\begin{array}{r}85 \\
124\end{array}$ & 218 & 705 & 70 & 706 & 698 & -0.8 & $\begin{array}{l}702 \\
702\end{array}$ & $\begin{array}{l}-0.6 \\
+3.8\end{array}$ & 218 & $\mid \begin{array}{c}0 \\
-1,0\end{array}$ & $\begin{array}{l}26 \\
31\end{array}$ & $\begin{array}{l}16.0 \\
21.6\end{array}$ & $\begin{array}{l}0.0 \\
4.3\end{array}$ & 17,3 & * P-4 & 8.5 & 2.0 & 6.5 & \\
\hline Gila & Saxton, Pa. & $8 \mathrm{w}$ & $\mid \begin{array}{r}124 \\
78\end{array}$ & $\begin{array}{l}299 \\
179\end{array}$ & $\begin{array}{l}675 \\
669\end{array}$ & $\begin{array}{l}677 \\
670\end{array}$ & $\begin{array}{l}676 \\
670\end{array}$ & $\begin{array}{l}694 \\
669\end{array}$ & $\begin{array}{l}+2.7 \\
-0.1\end{array}$ & 673 & & & & & & 1. & 8.6 & *State & 8.2 & -0.4 & 8.6 & *State \\
\hline & Tenn. & $9 \mathrm{w}$ & 140 & 3 & 620 & 61 & 620 & 619 & $\begin{array}{l}-0.1 \\
-0.2\end{array}$ & 624 & $\begin{array}{l}+0 . \\
+0 .\end{array}$ & 300 & $\mid \begin{array}{c}0.7 \\
-0.7\end{array}$ & 3 & 17 & 6. & 11.0 & $*$ *P-3 & & 0.7 & 11.5 & \\
\hline Win & , Wyo. & $10 \mathrm{~W}$ & 134 & 22 & 612 & 61 & 612 & 609 & -0.5 & 614 & +0. & 226 & +0.9 & & & 2.7 & 13.3 & -4 & 15.6 & 7.2 & 8.4 & \\
\hline Illix & h, Okla. & $11 \mathrm{~W}$ & 66 & $3 c$ & 5 & 5 & 5 & 566 & -0.9 & & 0 & 303 & +0.3 & 6 & & 2.5 & 0 & -3 & & 2.1 & 5.7 & \\
\hline & Onio & $12 \mathrm{~W}$ & 128 & 225 & 5 & 5 & 52 & 512 & -1.6 & & -0.6 & 222 & +1, & 28 & 21 & 5.1 & 16.2 & -5 & & 2.1 & 6.6 & \\
\hline Oconalufty & Cherok & $13 \mathrm{w}$ & 121 & 336 & 510 & 510 & 510 & 510 & & 512 & +0.4 & 336 & 0 & 34 & 24 & 10.0 & 14.0 & tHFA & 11.1 & 1.6 & 9.5 & *† $\mathrm{P}-2$ \\
\hline Penns & Penns C & $14 \mathrm{~W}$ & 114 & 237 & 454 & 454 & 454 & 464 & +2.2 & 468 & +3.1 & 234 & -1.3 & 30 & 19 & 2.0 & 17.3 . & *P-4 & 7.2 & 1.0 & 6.2 & $* \quad P-3$ \\
\hline & Md. & $76 \mathrm{~W}$ & 130 & 17 & 4 & 43 & 436 & 436 & 0 & 439 & +0 & & & 2 & 14 & 4.5 & 8. 7 & $*$ & 10.4 & 4.0 & 6.4 & * P-2 \\
\hline Prov & rk, Utah & $15 \mathrm{~W}$ & 102 & 185 & 4 & 43 & 430 & 427 & -0.7 & 433 & +0 . & 181 & $\mid \begin{array}{l}+0.0 \\
-2.2\end{array}$ & 2 & & 7.8 & 9.8 & * & & 2.9 & 3.8 & * SP-4 \\
\hline Big & ig Piney, N. D. & $16 \mathrm{~W}$ & 102 & 195 & 4 & $42-12 x-1$ & 42 & 440 & +3.0 & 442 & +3. & 195 & 0 & 26 & 15 & 2.2 & 13.0 & $* \dagger p-3$ & 16.5 & 3,5 & 13.0 & t SP-3 \\
\hline Ninr & Peck, Kans & $17 \mathrm{~W}$ & 134 & 20 & 3 & 39 & 39 & 380 & -3.8 & 382 & -3 & 204 & $|-1.9|$ & 28 & 15 & 5. & 9.8 & $*$ PP-2 & $13 . \mathrm{C}$ & 3.0 & 1c. $c$ & \\
\hline Colorado & Tex. & $18 \mathrm{~W}$ & 94 & 20 & 3 & $356^{\circ}$ & 35 & 356 & 0 & 358 & +o & 209 & 0 & 2 & 11 & 2. & 8.9 & $* i$ & 13.5 & 3.7 & 9.8 & $t \quad P-2$ \\
\hline Cheyenne & Was & $19 \mathrm{~W}$ & 82 & 18 & 347 & 347 & 34 & 348 & +0.3 & 352 & +1 & 182 & -0.5 & 2: & 10 & 3. & 7.5 & * & 8.6 & 2.7 & 5.9 & \\
\hline Milk & Crossing, Mont. & $20 w$ & 97 & 116 & 34 & 342 & 342 & 337 & -1. & 340 & & i) & $-0.9 \mid$ & 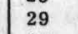 & & 8.1 & 6.6 & & 8.9 & 2.2 & 5.7 & \\
\hline South Tyger & Reiłville, S. C & $21 \mathrm{~W}$ & 93 & 202 & 339 & 339 & 339 & 335 & -1.2 & 335 & -1.2 & 200 & -1.0 & 27 & 19.0 & 6.3 & 12.7 & $* \uparrow \mathrm{P}-3$ & 14.5 & C.8: & 13.7 & * P-4 \\
\hline
\end{tabular}

* Experienced in mean-section method

*† Experienced in both methods

$\ddagger$ Used that portion measured from bridge 
TABLE 1 - Continued

\begin{tabular}{|c|c|c|c|c|c|c|c|c|c|c|c|c|c|c|c|c|c|c|c|c|c|c|}
\hline \multicolumn{2}{|c|}{ Gaging Station } & \multicolumn{6}{|c|}{ Special Measurement } & \multicolumn{7}{|c|}{ Normal Measurement } & \multicolumn{4}{|c|}{ Computation } & \multicolumn{4}{|c|}{ Checking } \\
\hline \multirow[b]{2}{*}{ River } & \multirow[b]{2}{*}{ Place } & \multirow[b]{2}{*}{$\begin{array}{l}\text { File } \\
\text { No. }\end{array}$} & \multirow[b]{2}{*}{$\begin{array}{l}\text { No. } \\
\text { Obs. }\end{array}$} & \multirow[b]{2}{*}{ Area } & \multirow[b]{2}{*}{$\begin{array}{l}\text { Mean- } \\
\text { Sec. }\end{array}$} & \multirow[b]{2}{*}{$\begin{array}{l}\text { Mid- } \\
\text { Sec. }\end{array}$} & \multirow[b]{2}{*}{ Average } & \multirow[b]{2}{*}{$\begin{array}{l}\text { Mean- } \\
\text { Sec. }\end{array}$} & & & & & & & Time & in Min & nutes & & Time & in $\operatorname{Min}$ & Lutes & \\
\hline & & & & & & & & & $\begin{array}{l}\text { cent } \\
\text { Diff. }\end{array}$ & $\begin{array}{l}\text { Mid- } \\
\text { sec. }\end{array}$ & \begin{tabular}{|l|} 
cent \\
Diff.
\end{tabular} & Area & $\begin{array}{l}\text { cent } \\
\text { Diff. }\end{array}$ & \begin{tabular}{|l} 
No. \\
Obs.
\end{tabular} & \begin{tabular}{|r} 
Mean- \\
Sec. \\
\end{tabular} & Diff. & \begin{tabular}{|l} 
Mid- \\
Sec.
\end{tabular} & Grade & $\begin{array}{c}\text { Mean- } \\
\text { Sec. }\end{array}$ & Diff. & \begin{tabular}{|l|} 
Mid- \\
Sec.
\end{tabular} & Grade \\
\hline Smith & Philpott, Va. & $22 \mathrm{~W}$ & 91 & & 334 & 334 & 334 & 328 & -1.8 & 330 & $-1,2$ & & -1.5 & 25 & 17.9 & 4.3 & 13.6 & P-2 & 10.1 & 0.6 & 9.5 & * P -2 \\
\hline Peco & Puerto de Luna, N.M. & $23 \mathrm{w}$ & 135 & 126 & 302 & 302 & 302 & 299 & $\mid \begin{array}{l}-1.0 \\
-1.0\end{array}$ & 300 & -0.7 & 126 & 0 & 30 & 19.6 & 3.8 & 15.8 & $P-4$ & 10.4 & 3.1 & 7.3 & \\
\hline Powder & Arvada, Wyo. & $24 \mathrm{~W}$ & 109 & 92.4 & 298 & 2 & 298 & 294 & -1.3 & 295 & -1.0 & 918 & -0.6 & 25 & 11.3 & 3.5 & 7.8 & $P-2$ & 15.6 & 8.4 & 7.2 & $\dagger \quad P-2$ \\
\hline Farmington & Riverton, Conn. & $25 \mathrm{~W}$ & 88 & 306 & 289 & 289 & 289 & 287 & $-0.7 \mid$ & 289 & 0 & 302 & 7.3 & 29 & 18.5 & 4.9 & 13.6 & P-2 & 8.6 & 1.4 & 7.2 & * $\quad$ P-3 \\
\hline Cedar & East Lansing, Mich, & $26 \mathrm{~W}$ & 109 & 209 & 279 & 28 & 280 & 280 & 0 & 284 & +1.4 & 207 & $\mid-1.0$ & 27 & 9.9 & 4.2 & 5.7 & $x+P-3$ & 12.0 & 4.2 & 7.8 & $\begin{array}{ll}t & P-2\end{array}$ \\
\hline Farmington & New Boston, Mass. & $27 \mathrm{w}$ & 101 & 130 & 280 & 281 & 281 & 265 & $|-5.7|$ & 272 & $|-2.9|$ & 127 & +2.3 & 30 & 22.2 & 6.7 & 15.5 & $P-4$ & 9.2 & 1.7 & 7.5 & * P-3 \\
\hline w. Fork San Jacinto & Humble, Tex. & $28 \mathrm{~W}$ & 114 & 244 & 274 & 274 & 274 & 271 & -1.1 & 272 & -0.7 & 245 & +0.4 & 27 & 10.0 & 2.7 & 7.3 & $P-3$ & 12.0 & 3.8 & 8.2 & * SP-4 \\
\hline Little Pigeon & Sevierville, Tenn. & $29 \mathrm{~W}$ & 78 & 205 & 272 & 271 & 272 & 270 & $-0.7 \mid$ & 272 & 0 & 209 & +1.9 & 23 & 10.0 & 2.0 & 8.1 & $P-3$ & 8.5 & 1.7 & 6.8 & *State \\
\hline San Joaquin & Fre. Ford Br., Calif. & $30 \mathrm{w}$ & 85 & 302 & 260 & 260 & 260 & 261 & +0.4 & 263 & +1.2 & 299 & -1.0 & 24 & 18.0 & 5.8 & 12.2 & & & 1.4 & 6.1 & $* \mathrm{SP}-4$ \\
\hline No. Canadian & El Reno, Okla. & $31 \mathrm{w}$ & 77 & 153 & 244 & 24 & 244 & 241 & {$\left[\begin{array}{cc}0 \\
-1\end{array}\right.$} & 244 & 0 & 153 & 0 & 23 & 13.3 & 2.9 & 10.4 & & & 1.6 & 6.6 & $2-3$ \\
\hline Little Coal & W. Va. & $32 \mathrm{~W}$ & 119 & 239 & 240 & 24 & 240 & 237 & $|-1.2|$ & 238 & -0.8 & 235 & $|-1.7|$ & 27 & 12.5 & 3.5 & 9.0 & P-1 & 8.6 & 2.6 & 6.0 & $P-2$ \\
\hline Guadalupe & unfels, Tex. & $33 \mathrm{~W}$ & 113 & 258 & 234 & 234 & 234 & 231 & $|-1.3|$ & 231 & $|-1.3|$ & 257 & $|-0.4|$ & 27 & 23.0 & 9.0 & 14.0 & SP-5 & 9.0 & 2.5 & 6.5 & THFA \\
\hline Reed Creek & Grahams Forge, Va. & $34 \mathrm{~W}$ & 111 & 246 & 232 & 232 & 232 & 230 & $|-0.9|$ & 230 & $-0.9 \mid$ & 242 & $|-1.6|$ & 29 & 18.8 & $\mid-4,2$ & 23.0 & SP-5 & 12.9 & 3.7 & 9.2 & * SP-7 \\
\hline Zumbro & Zumbro Falls, Minn. & $35 \mathrm{~W}$ & 76 & 105 & 224 & 224 & 224 & 224 & 0 & 224 & 0 & 105 & 0 & 27 & 12.8 & 1.8 & 11,0 & $P-2$ & 14.6 & 3.8 & 10.8 & $* \dagger P-4$ \\
\hline Mills & Mills River, N. G. & $36 \mathrm{~W}$ & 85 & 121 & 205 & 205 & 205 & 202 & $\mid-1.5$ & 203 & $|-1.0|$ & 121 & 0 & 26 & 11.8 & 5.0 & 6.8 & $\mathrm{P}-3$ & 11.9 & 4.8 & 7.1 & if $\mathrm{SP}-6$ \\
\hline Tobesofkee & Macon, Ga. & $37 \mathrm{~W}$ & 78 & 133 & 202 & 202 & 202 & 201 & $|-0.5|$ & 203 & +0.5 & 134 & +0.7 & 23 & 11 & 0.7 & 10.6 & $\mathrm{P}-3$ & & 1.4 & 3.8 & $P-4$ \\
\hline Animas & , Colo & $77 \mathrm{~W}$ & 127 & 93.4 & 177 & 177 & 177 & 161 & -9. & 160 & $\mid-9.6$ & 91.2 & -2.4 & 31 & 21 & 7.1 & 13.9 & $\mathrm{P}-2$ & & 1.5 & 6.7 & $\mathrm{P}-3$ \\
\hline Casselman & $\mathrm{a}, \mathrm{Pa}$. & $38 \mathrm{w}$ & 136 & 201 & 202 & 201 & 2 & 188 & -6.9 & 190 & $|-6.0|$ & 197 & -2.0 & 28 & 9.7 & 0.4 & 9.3 & $P-4$ & 8.6 & -0.1 & 8.7 & $P-4$ \\
\hline Homochitto & Eddiceton, Miss. & $39 \mathrm{w}$ & 97 & 121 & 189 & 189 & 189 & 186 & $\mid-1.6$ & 186 & $\mid-1,6$ & 120 & +0.8 & 28 & 15.4 & 1.7 & 13.7 & $P-1$ & 9.9 & 2.5 & 7.4 & $P-2$ \\
\hline Wallkill & Unionville, $\mathrm{N}$. Y. & $40 \mathrm{~W}$ & 103 & 101 & 179 & 178 & 178 & 175 & -1.7 & 175 & $-1.7 \mid$ & 102 & +1.0 & 28 & 15.7 & 5.3 & 10.4 & P-3 & 6.4 & 0 & 6.4 & * $\quad P-4$ \\
\hline Tickfaw. & Holden, La. & $41 \mathrm{~W}$ & 93 & 111 & 165 & 166 & 166 & 167 & +0.6 & 168 & +1.2 & 111 & 0 & 25 & 15.3 & 2.8 & 12.5 & P-4 & 12.1 & 4.8 & $7.3=$ & $*{ }_{1} P-3$ \\
\hline San Antonio & Falls City, Tex. & $42 W$ & 92 & 112 & 162 & 162 & 162 & 155 & -4.3 & 158 & -2.5 & 110 & $\mid-1.8$ & 2 & 8 & 2.0 & 6.8 & $P-3$ & & & 4.8 & if $\mathrm{SP}-4$ \\
\hline Richland & Dayton, Tenn. & $43 \mathrm{~W}$ & 91 & 84,0 & 156. & 15 & 156 & 157 & +0 & 1 & 0 & $\begin{array}{r}84.9 \\
\end{array}$ & +1.1 & 26 & & 6.5 & 11.5 & $P-2$ & 9.0 & 2.0 & 7.0 & $P-3$ \\
\hline Blackfoot & Blackfoot, Idaho & $44 W$ & 79 & 70.5 & 15 & 1 & & 151 & +0.7 & 152 & +1.3 & 70,2 & -0.4 & 20 & 3.9 & 6.1 & 7.8 & $\mathrm{SP}-4$ & 4.1 & 1.6 & 2.5 & $S P-4$ \\
\hline Still & Robertsville, Conn. & $45 \mathrm{~W}$ & 88 & 114 & 149 & 149 & 149 & 154 & +3.2 & 153 & +2.7 & 115 & +0.9 & 29 & 15.0 & 1.0 & 14.0 & P-3 & 12.1 & 5.4 & 6.7 & tHFA \\
\hline E. Walker & Yerington, Nev. & $46 \mathrm{~W}$ & 116 & 70,8 & 1 & 14 & 14 & 146 & +0.7 & 147 & +1.4 & 70.8 & 0 & 29 & 21.1 & 3.7 & 17.4 & Sta & $14.8^{\circ}$ & 5,6 & 9.2 & $* \quad P-3$ \\
\hline Fall & Ithaca, $\mathbf{N} ; \mathbf{Y}$. & 47 & 102 & 64.8 & 1 & 14 & 14 & 142 & -0.7 & 142 & $-0.7 \mid$ & 64.4 & -0.6 & 27 & 12.5 & 1.9 & 10.6 & P-3 & 10.4 & 3.4 & 7.0 & $P-3$ \\
\hline Bean Blosson & Do & 48 & 99 & 73,4 & 142 & 1 & 142 & 140 & -1.4 & 142 & 0 & 73.2 & +0.3 & 25 & 11.3 & 3.4 & 7.9 & $\mathrm{P}-2$ & 8.5 & 2.5 & 6.0 & $\mathrm{P}-1$ \\
\hline No. Br. Rancocas & Pemberton, N. J. & $49 \mathrm{~W}$ & 97 & 150 & 140 & 140 & 140 & 139 & -0.7 & 139 & $-0.7 \mid$ & 148 & -1.3 & 25 & 15. & 8.0 & 7.5 & -2 & 9.8 & & 6.0 & $\mathrm{P}-3$ \\
\hline Pequest & Pequest, N. J. & $50 \mathrm{~W}$ & 91 & 107 & 137 & 137 & 137 & 134 & -2.2 & 135 & -1.5 & 107 & 0 & 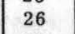 & 16 & 6.8 & 10.0 & t SP-6 & 12.0 & 3.5 & 8.5 & $\mathrm{P}-2$ \\
\hline Gales & Forest Grove, Oreg. & $51 \mathrm{~W}$ & 79 & 111 & 13 & & 1 & 132 & 0 & 133 & +0.8 & 110 & +1.0 & 22 & 8.5 & 0.8 & 7.7 & $P-2$ & 6.6 & -0.1 & 6.7 & P-1 \\
\hline Austin & Binghan & $52 \mathrm{~W}$ & 88 & 92.8 & & 118 & 1 & 118 & 0 & 119 & +0.9 & 92.4 & -0.4 & 23 & 11.6 & 4.5 & 7.1 & $P-1$ & 4.2 & 1.0 & 3.2 & $P-3$ \\
\hline So. Br. Waits & Bradford, vt. & $53 \mathrm{~W}$ & 102 & 49.7 & 115 & 115 & 11 & 11 & +3.4 & 120 & +4.3 & 49.7 & 0 & 28 & 13.5 & 2.5 & 11.0 & $\mathrm{P}-4$ & 11.9 & 6.3 & 5.6 & $\mathrm{SP}-4$ \\
\hline English & Kalona, Iowa & $54 \mathrm{~W}$ & 82 & 120 & 113 & 113 & 113 & 113 & 0 & 114 & +0.9 & 120 & 0 & 22 & 13.0 & 1.5 & 11.5 & $\mathrm{P}-2$ & 9.4 & 2.4 & 7.0 & $\mathrm{SP}-5$ \\
\hline Cannon & Welch, Minn. & $55 \mathrm{~W}$ & 77 & 91.8 & 103 & 103 & 103 & 101 & -2.0 & 102 & -1.0 & 91.3 & -0.5 & 28 & 14.2 & 3.2 & 11.0 & P-2 & 13.0 & 2.0 & 11.0 & *t $P-4$ \\
\hline Pecatonica & Darlington, Wis. & $56 \mathrm{~W}$ & 89 & 63.2 & 102 & 102 & 102 & 100 & -2.0 & 101 & -1.0 & 62.1 & -1.7 & 27 & 13.7 & 4.7 & 9.0 & $\mathrm{P}-2$ & 7.6 & 0.6 & 6.4 & $P-3$ \\
\hline Laurel Hill & Ursi & 57 & 126 & 78.6 & 96.2 & 96.1 & 96.2 & 96.0 & -0.2 & 96.3 & +0.1 & 77.8 & -1.0 & 29 & 12.4 & 0.4 & 12.0 & $P-4$ & 8.9 & $-2,8$ & 11.7 & $P-4$ \\
\hline Sangamon & Mahomet, IIl. & $58 \mathrm{~W}$ & 80 & 102 & 88,6 & 88.8 & 88. & 87.5 & -1.4 & 88.4 & $|-0.3|$ & 97.9 & -4.0 & 25 & 9.5 & 1.3 & 8.2 & P-2 & 8.5 & 0.9 & 7.6 & $\mathrm{P}-1$ \\
\hline Busseron & Carlisle, Ind. & $59 \mathrm{~W}$ & 103 & 44.2 & 86.3 & 86. & 86. & 87.5 & +1.3 & 88.1 & $+2,0$ & 44.0 & $\mid-0.5$ & 28 & 12.1 & 1.9 & 10.2 & $P-1$ & 10.1 & 3.3 & 6.8 & P-2. \\
\hline Petit Jean & Booneville, Ark. & $60 \mathrm{~W}$ & 79 & 54,0 & 81.0 & 81.1 & 81.0 & 80.0 & -1.2 & 80.7 & -0.5 & 53.9 & -0.2 & 25 & 19.8 & 2.9 & 16.9 & $P-2$ & 12.4 & 3.1 & 9.3 & $S P=5$ \\
\hline Saint Mary's & Md. & 78 & 118 & & 94 & & 94 & 73.6 & -0.7 & 74.4 & +0.4 & 32.0 & -0.6 & 29 & 13.2 & 3.1 & 10.1 & $P-2$ & 13.0 & 3.6 & 9.4 & $P-3$ \\
\hline Kayaderosseros & W. Milton, N. Y. & $61 \mathrm{~W}$ & 98 & 97.0 & 73.5 & 73 & 73 & 72.7 & -1.2 & 72.9 & -1.0 & 96.0 & -1.0 & 32 & 16.0 & 3.4 & 12.6 & P-3 & 7.2 & 0.6 & 6.6 & $P-4$ \\
\hline Santa Ana & Prado Dam, Calif. & $62 W$ & 63 & 21.4 & 70.0 & 70.1 & 70.0 & 70.4 & +0.6 & 70.7 & +1.0 & 21.4 & 0 & 18 & 5.6 & 0.6 & 5.0 & $\mathrm{P}-3$ & 6.9 & 1.1 & 5.8 & $\mathrm{P}-2$ \\
\hline San ta Ana & Prado, Calif & $63 \mathrm{~W}$ & 108 & 43.4 & 69.3 & 69.6 & 69.4 & 67.7 & -2.4 & 68.7 & $-1.0 \mid$ & 43.0 & -0.9 & 28 & 18.0 & 8.5 & 9.5 & P-2 & 9.9 & 0.1 & 10.0 & $+1 \mathrm{P}-4$ \\
\hline
\end{tabular}

-section method
-section method

$t^{*}$ Experienced in mid-section
$* \dagger$ Experienced in both methods 


\begin{tabular}{|c|c|c|c|c|c|c|c|c|c|c|c|c|c|c|c|c|c|c|c|c|c|c|}
\hline \multicolumn{2}{|c|}{ Gaging Station } & & \multicolumn{5}{|c|}{ Special Measurement } & \multicolumn{7}{|c|}{ Normal Measurement } & \multicolumn{4}{|c|}{ Computation } & \multicolumn{4}{|c|}{ Checking } \\
\hline \multirow{2}{*}{ River } & \multirow{2}{*}{ Place } & \multirow[b]{2}{*}{$\begin{array}{l}\text { File } \\
\text { No. }\end{array}$} & \multirow[b]{2}{*}{$\begin{array}{l}\text { No. } \\
\text { Obs. }\end{array}$} & \multirow{2}{*}{ Area } & \multirow{2}{*}{$\begin{array}{c}\text { Mean- } \\
\text { Sec. }\end{array}$} & \multirow[b]{2}{*}{$\begin{array}{c}\text { Mid- } \\
\text { Sec. }\end{array}$} & \multirow{2}{*}{ Average } & \multirow[b]{2}{*}{$\begin{array}{l}\text { Mean- } \\
\text { Sec. }\end{array}$} & \multirow{2}{*}{\begin{tabular}{|l|} 
Per- \\
cent \\
Diff. \\
\end{tabular}} & & & & & & Time & in $\mathrm{Min}$ & untes & & Time 1 & in $\mathrm{Min}$ & lutes & \\
\hline & & & & & & & & & & $\begin{array}{l}\text { Mid- } \\
\text { Sec. }\end{array}$ & $\begin{array}{l}\text { cent } \\
\text { Diff. }\end{array}$ & Area & Diff. & $\begin{array}{l}\text { No. } \\
\text { Obs. }\end{array}$ & $\begin{array}{c}\text { Mean- } \\
\text { Sec. }\end{array}$ & Diff. & $\begin{array}{c}\text { Mid- } \\
\text { Sec. }\end{array}$ & Grade & $\begin{array}{c}\text { Mean- } \\
\text { Sec. }\end{array}$ & Diff. & $\begin{array}{r}\text { Mid- } \\
\text { Sec }\end{array}$ & Grade. \\
\hline Elkhorn & Fr & $64 \mathrm{I}$ & 108 & 65.4 & 66.7 & 6 & 66.6 & 66.1 & -0.8 & 66.5 & $-0.1 \mid$ & 65.8 & +0.6 & 28 & 6.6 & $|-0.2|$ & 6.8 & & 6.6 & 2.0 & 4.6 & \\
\hline Warm & & & & & & & & 58.9 & $-0.3 \mid$ & 59.2 & & 28.15 & -0.5 & 18 & 10.8 & -0.2 & 11.0 & $* \dagger$ P-2 & 6.2 & 2.0 & 4.2 & \\
\hline Hanapepe & Koula, Hawaii & $66 \mathrm{~W}$ & 59 & 91.3 & 51.0 & 5 & 51.2 & 49.8 & -2.7 & 50.6 & -1. & 90.4 & $|-1.0|$ & 34 & 19.8 & 9.9 & 9.9 & * SP-4 & 11.2 & 4.5 & 6.7 & *State \\
\hline Little Camas Canal & Bennett, Idaho & $67 \mathrm{w}$ & 116 & 29.7 & 45.2 & 45.2 & 45.2 & 45.1 & -0.2 & 45,4 & +0.4 & 29.3 & $|-1.3|$ & 28 & 17.9 & 1.3 & 16.6 & *State & 8.6 & 2.1 & 6.5 & * SP-4 \\
\hline Withlacooche & Trilb & $68 \mathrm{w}$ & 90 & 66.0 & 44.3 & 44.3 & 44.3 & 44.0 & +0.7 & 44.0 & +0.7 & 66.0 & 0 & 26 & 22.3 & 6.6 & 15.7 & tHFA & 11.3 & 2.3 & 9.0 & \\
\hline Eno & Hillsboro, N. C. & $69 \mathrm{w}$ & 93 & 46.5 & 44.1 & 44.0 & 44.0 & 44.0 & 0 & 44.2 & +0.5 & 45.5 & $|-2.1|$ & 28 & 21.5 & 6.8 & 14.7 & $\mid 7 \mathrm{SP}-6$ & 8.1 & 1.5 & 6.6 & $\mathrm{P}-3$ \\
\hline North Concho & San Angelo, Tex. & $70 \mathrm{w}$ & 75 & 21.4 & 35.3 & 35.3 & 35. & 35.4 & +0.3 & 35,5 & +0.6 & 21.3 & $\left|\begin{array}{r}-0.5 \\
-0.5\end{array}\right|$ & 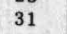 & 23.0 & 9.7 & 13.3 & $\begin{array}{l}\dagger \\
\mathrm{P}-2\end{array}$ & 11.5 & 5.0 & 6.5 & \\
\hline Warm. & lif & $71 \mathrm{~W}$ & 56 & 20.7 & 33.0 & 33. & 33.0 & 32.7 & $-0.9 \mid$ & 32.9 & $|-0.1|$ & 20. 7 & 0 & 22 & 12.5 & 5.4 & 7.1 & $\mid *+\quad P-2$ & 6.8 & 2.0 & 4.8 & $P-3$ \\
\hline Tub Springs & Ne & $79 w$ & 58 & 20.8 & 30.2 & 30.3 & 30.25 & 29.9 & -1.2 & 30.5 & +0.8 & 20.3 & -2.4 & 15 & 5.4 & 0.8 & 4.6 & $* \quad P-4$ & 5.3 & 7 & 3.6 & \\
\hline Evitts & ey, Pa & $80 w$ & 118 & 24.5 & 25.9 & 25.9 & 25.9 & 26.4 & +1.9 & 26.4 & +1.9 & 24.7 & +0.8 & 27 & 9.8 & 1.5 & 8.3 & $\begin{array}{lll}* & \mathrm{P}-3\end{array}$ & 6.1 & .5 & 5.6 & \\
\hline Green & Glads & $72 \mathrm{w}$ & 92 & 19.7 & 18.6 & 18.6 & 18. 6 & 18.65 & +0.3 & 18.7 & +0.5 & 19.55 & $5-0.8$ & 28 & 12.3 & 3.2 & 9.1 & 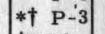 & 10.8 & 2.6 & 8.2 & $+\mathrm{SP}-5$ \\
\hline Honokohau & Honokahau, Hawaii & $73 \mathrm{w}$ & 45 & 35.3 & 17.0 & 17.0 & 17.0 & 17.0 & 0 & 16.9 & -0.6 & 35.05 & -0.7 & 23 & 18.2 & 1.4 & 16.8 & tHFA & 8.5 & 0.6 & 7.9 & * P-3 \\
\hline Kahakuloa & Honokahau, Hawaii & $74 \mathrm{~W}$ & 53 & 27.6 & 8. 32 & 8.33 & 8.32 & 8.44 & +1.3 & 8.46 & +1.7 & 28.2 & $\mid+2.2$ & 27 & 20.3 & $\mid-4.3$ & 24.6 & *State & $13.1^{\circ}$ & 3.1 & 10.0 & $* \dagger P-3$ \\
\hline & & & & & & & & $3 L$ & & MI $>$ & 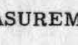 & & & & & & & & & & & \\
\hline Columbia & Trinid & 1C & 119 & 16,642 & 57,324 & & & 57310 & 0 & 0 & 10.5 & 32 & & 32 & 26.0 & & 18.6 & P-1 & & 3,4 & $\mid 11.7$ & \\
\hline Missouri $\ddagger$ & Ft. Randall Dam, S. D. & $2 \mathrm{C}$ & 100 & 11,240 & 26,970 & 27,039 & 27,004 & $27 ; 060$ & +0.2 & 27,350 & +1.3 & 11,271 & +0.3 & 28 & 22.6 & 4.9 & 17.7 & * $\quad \mathrm{P}-1$ & 11.7 & 1.7 & 10.0 & \\
\hline Arkansas & Van B & $3 \mathrm{C}$ & 86 & 7,260 & 22,950 & 22,962 & 22,956 & 22,549 & -1.8 & 22,696 & $\mid-1.1$ & 7,240 & $|-0.3|$ & 34 & 32.5 & 13.1 & 19.4 & $\begin{array}{ll}* & \mathbf{P}-2\end{array}$ & 18.0 & 0.9 & 17.1 & -7 \\
\hline Snake & Weis & $4 \mathrm{C}$ & 104 & 6,568 & 21,896 & 21,911 & 21 , & 21, & -0.2 & & +0.2 & 32 & & 20 & 17.7 & & 16.6 & & 0 & 6.8 & 6.2 & \\
\hline Colorado & $\mathrm{L}$ & $5 \mathrm{C}$ & 82 & 3,053 & 21,095 & 21,108 & 98 & 20,913 & -0.9 & 21,042 & -0.3 & 3,016 & -1.2 & 24 & 30.3 & 8.1 & 22.2 & te & 10.4 & -3.3 & 13.7 & \\
\hline Muskingum & ville, Ohio & 6C & 84 & 7,260 & 12,036 & 12,044 & 12,044 & 11,883 & -1.3 & 11,998 & -0.4 & 7,202 & -0.8 & 25 & 18.2 & 4.3 & 13.9 & $* \quad P-1$ & 14.2 & 3.4 & 10.8 & * P-2 \\
\hline Coloradó & Hit & $7 \mathrm{C}$ & 124 & 3,724 & 11,246 & 11,250 & 11,248 & 11,079 & -1.5 & 11,182 & -0.6 & 3,691 & $|-0.9|$ & 31 & 22.6 & 7.2 & 15.4 & * SP-4 & 7.6 & 2.3 & 5.3 & * SP-4 \\
\hline Brazos & mond, Tex. & $8 \mathrm{C}$ & 79 & 3,688 & 10,101 & 10,135 & 10,118 & 10,010 & $\mid-1.1$ & 10,071 & -0.5 & 3,671 & -0.5 & 29 & 30.0 & 13.5 & 16.5 & $\mid+\mathrm{SP}-5$ & 9.6 & -1.2 & 10.8 & \\
\hline Payette & Horseshoe Bend, Idaho & $9 \mathrm{C}$ & 118 & 1,868 & 10,046 & 10,049 & 10,048 & 9,937 & -1.1 & 9,986 & -0.6 & 1,852 & -0. & 28 & 13,7 & 4 & 9.7 & *State & 9.5 & 3.4 & 6.1 & \\
\hline Merrimack & Lowell, Mass. & $10 \mathrm{C}$ & 125 & 4,275 & 9,926 & 9,949 & 9,938 & 9,8 & -0 & 9 , & +0 & 4, & 0 & 36 & 34.5 & & 24 & $\mid \begin{array}{ll}\mathrm{P}-2 \\
\end{array}$ & & & 9.4 & \\
\hline Wabash $\ddagger$ & River & $11 \mathrm{C}$ & 99 & 4,418 & & 9, & & & -0 & & -0 & 4, & $|-0.7|$ & 28 & 20.2 & 5.2 & 15.0 & {$\left[\begin{array}{ll}* & P-1\end{array}\right.$} & 2.0 & 0.2 & 11.8 & $* \quad \mathrm{P}-2$ \\
\hline Puyallup & Wash. & $12 \mathrm{C}$ & 120 & 1,622 & 8,280 & 8,284 & 8,282 & 8,229 & -0.6 & 8,275 & -0.1 & 1,615 & -0.4 & 28 & 16.7 & 6.9 & 9.8 & * $\quad \mathrm{P}-1$ & 10.2 & 3.7 & 6.5 & \\
\hline Neosho & on, Okla. & $13 \mathrm{C}$ & 77 & 2,797 & 8,092 & 8,105 & 8,098 & 7, 977 & -1.5 & 8,022 & -0 & 2,781 & $\mid-0.6$ & 25 & 15.0 & & 12.5 & & 7.3 & 1.9 & 5.4 & \\
\hline Snake & Idat & & 180 & 2, & & 7, & & & -1. & 7,8 & -1.3 & & +0 & 46 & 28.7 & 9.5 & 19.2 & * SP-4 & 19.5 & 2.1 & 17.4 & \\
\hline Brazos & Te & & 93 & 2,2 & & 7,0 & & 7, & +0.1 & 7,020 & +0.1 & 2, & -1.4 & 26 & 23,1 & 2 & 21.1 & $t \quad P-2$ & 9.6 & 1.4 & 8.2 & \\
\hline Broad & s. c & & 110 & 3,375 & 6.7 & 6,785 & 6,785 & 6,706 & -1.2 & 6,697 & -1.3 & 3, & -2.2 & 29 & 14.3 & 3 & 10 & $*+\mathrm{P}$ & 9.6 & -0.7 & 10.3 & \\
\hline Chenango & Chenango Forks, N. Y. & $17 \mathrm{C}$ & 113 & 1,858 & 6,525 & 6,536 & 6,530 & 6,537 & +0. & 6,605 & +1.1 & 1,8 & -0 & 30 & 19.2 & 7 & 12 & * $\quad$ P-3 & 8.6 & -6.3 & 8.9 & * $\mathrm{P}-4$ \\
\hline San Juan & Farmington, N. M. & $18 \mathrm{C}$ & 113 & 1,028 & 6,440 & 6,440 & 6,440 & 6,3 & -0.8 & 6,401 & -0. & & -0.2 & & 19.9 & & 17.8 & $\dagger \mathrm{P}-4$ & 4.7 & 3.4 & 11.3 & -1 \\
\hline French Broad & Hot Springs, N. C. & $19 \mathrm{C}$ & 101 & 1,555 & 5,6 & 5,6 & 5,613 & 5,5 & -0.9 & 5,611 & 0 & 1,530 & -1.6 & 27 & 15.9 & 3.9 & 12.0 & *† $\mathrm{P}-2$ & 11.0 & 4.0 & 7.0 & \\
\hline Brazos & D & $20 \mathrm{C}$ & 93 & 1,706 & 4,674 & 4,6 & 4,6 & $4, E$ & $-0.3 \mid$ & 4,6 & +0.3 & & -0.2 & 2 & & & 9.2 & & 14.0 & 4.0 & 10.0 & $\dagger \mathrm{H}$ \\
\hline Flathead & c. & 21 & 87 & $2, ?$ & & & & & -0 & 4 & to. & & -0 & $2,2>$ & & & 11.8 & & 12.2 & 2.4 & 9.8 & \\
\hline Che & w. Va. & 22 & 125 & $1, ?$ & 4,3 & 4, & & 4,280 & $-0.8 \mid$ & 4,316 & 0 & 1,697 & -0.4 & 29 & 14.5 & -2.4 & 16.9 & $D_{0}$ & 11.4 & 2.6 & 8.8 & \\
\hline Shenandoah & lville, w. Va. & $38 \mathrm{C}$ & 152 & 2,189 & 4,013 & 4,020 & 4,016 . & 4,040 & +0.6 & 4,050 & +0.8 & 2,168 & -1.0 & 33 & 21.9 & 7.1 & 14.8 & & 14.3 & 3.9 & 10.4 & * P-3 \\
\hline 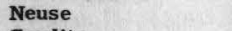 & N. C. & $23 \mathrm{C}$ & 87 & 1,830 & 3,932 & 3,933 & 3,932 & 3,872 & -1.5 & 3,896 & -0.9 & 1,822 & -0.1 & 27 & 21.0 & & & $\mid t$ SP-6 & 7.8 & 2.6 & 5.2 & $* \dagger \quad \mathrm{P}-3$ \\
\hline Cowlitz & Kosmos, Was & $24 \mathrm{C}$ & 110 & 1,244 & 3,905 & 3, 898 & 3,902 & 3,8 & -1.4 & 3,871 & -0.8 & 1,242 & -0.2 & 29 & 24.3 & 7.6 & 16.7 & * P-3 & 8.6 & 2.6 & 6.0 & \\
\hline Snake & Milner, Idaho & $25 \mathrm{C}$ & 131 & 2,039 & 3,893 & 3,889 & 3, 891 & 3,960 & +1.8 & 3,946 & +1.4 & 2,031 & -0.4 & 39 & 21.4 & 5.0 & 16.4 & -4 & 22.0 & 3.2 & 18.8 & \\
\hline anislaus & Calif. & $26 \mathrm{C}$ & 95 & 1,585 & 3,617 & 3,6 & 3,6 & 3,552 & -1.8 & 3,606 & -0.3 & 1,588 & +0.2 & 28 & 18.6 & 1.3 & 17.3 & $*$ St & 12.9 & 3.3 & 9.6 & * SP-4 \\
\hline Deep : & Moncure, N. C. & $27 \mathrm{C}$ & 130 & 2,045 & 3,570 & 3,568 & 3,569 & 3,548 & -0.6 & 3,553 & -0.4 & 2,050 & +0.2 & 35 & 22.0 & 9.5 & 12.5 & t SP-6 & 11.4 & 2.6 & 8.8 & *t $\mathrm{P}$ \\
\hline Gasconade & Jerome, Mo. & $28 \mathrm{C}$ & 91 & 986 & 3,500 & 3,504 & 3,502 & 3,482 & -0.6 & 3,509 & +0.2 & 963 & -2.4 & 29 & 21.7 & 3.9 & 17.8 & tHFA & 29.0 & 16.0 & 13.0 & THFA \\
\hline
\end{tabular}

* Experienced in mean-section method

*t Experienced in both methods

t Boat Measurements 
TABLE 1 - Continued

\begin{tabular}{|c|c|c|c|c|c|c|c|c|c|c|c|c|c|c|c|c|c|c|c|c|c|c|}
\hline \multicolumn{2}{|c|}{ Gaging Station } & & \multicolumn{5}{|c|}{ Special Measurement } & \multicolumn{7}{|c|}{ Normal Measurement } & \multicolumn{4}{|c|}{ Computation } & \multicolumn{4}{|c|}{ Checking } \\
\hline \multirow[b]{2}{*}{ River } & \multirow[b]{2}{*}{ Place } & \multirow[b]{2}{*}{$\begin{array}{l}\text { File } \\
\text { No. }\end{array}$} & \multirow[b]{2}{*}{$\begin{array}{l}\text { No. } \\
\text { Obs. }\end{array}$} & \multirow[b]{2}{*}{ Area } & \multirow[b]{2}{*}{\begin{tabular}{|l} 
Mean'- \\
Sec.
\end{tabular}} & \multirow[b]{2}{*}{$\begin{array}{l}\text { Mid- } \\
\text { Sec. }\end{array}$} & \multirow[b]{2}{*}{ Average } & \multirow[b]{2}{*}{$\begin{array}{l}\text { Mean- } \\
\text { Sec. }\end{array}$} & \multirow{2}{*}{\begin{tabular}{|l|} 
Per- \\
cent \\
Diff.
\end{tabular}} & \multirow[b]{2}{*}{$\begin{array}{l}\text { Mid- } \\
\text { Sec. }\end{array}$} & & & & & Time & in $\mathrm{Mir}$ & zutes & & Time & in Min & útes & \\
\hline & & & & & & & & & & & $\begin{array}{l}\text { cent } \\
\text { Diff. }\end{array}$ & Area & $\begin{array}{l}\text { cent } \\
\text { Diff. }\end{array}$ & $\begin{array}{l}\text { No. } \\
\text { Obs. }\end{array}$ & $\begin{array}{c}\text { Mean- } \\
\text { Sec. } \\
\end{array}$ & Diff. & \begin{tabular}{|l|}
$\begin{array}{l}\text { Mid- } \\
\text { Sec. }\end{array}$ \\
\end{tabular} & Grade & \begin{tabular}{|c}
$\begin{array}{r}\text { Mean- } \\
\text { Sec. }\end{array}$ \\
\end{tabular} & Diff. & $\begin{array}{c}\text { Mid- } \\
\text { Sec. }\end{array}$ & Grade \\
\hline Tuolumme & LaGrange, Calif. & $29 \mathrm{C}$ & 90 & 893 & 3,285 & 3,284 & 3,284 & 3,270 & -0.4 & 3,276 & -0.2 & 890 & $-0.3 \mid$ & 28 & 22.3 & 7.8 & 14.5 & $\left|\begin{array}{ll}\dagger & \mathrm{P}-2\end{array}\right|$ & 10.3 & 3.7 & 6.6 & * $\mathrm{P}-3$ \\
\hline Gauley & Belva, W. Va. & $30 \mathrm{C}$ & 133 & 1,846 & 3,187 & 3,199 & 3,193 & 3,153 & -1.3 & 3,154 & -1.2 & 1,836 & -0.5 & 30 & $\cdot 13.5$ & 0.8 & .12 .7 & State & 11.2 & 2.2 & 9.0 & te \\
\hline Bayou Macon $t$ & Delhi, La. & $31 \mathrm{C}$ & 81 & 3,684 & 3,174 & 3,172 & 3,173 & 3,117 & -1.8 & 3,131 & -1.3 & 3,695 & +0.3 & 26 & 10.5 & 3.0 & 7.5 & P-3 & 11.1 & 2.8 & 8.3 & * P-3 \\
\hline Snake & Heise, Idaho & $32 \mathrm{C}$ & 150 & 1,023 & 2,928 & 2,931 & 2,930 & 2,945 & +0.5 & 2,950 & +0.7 & 1,022 & -0.1 & 39 & 15.1 & 5.8 & 9.3 & $\left|\begin{array}{ll}* \mathrm{SP} & -4\end{array}\right|$ & 10.8 & 1.3 & 9.5 & *State \\
\hline Cumberland & Cumberland Falls, Ky. & $33 \mathrm{C}$ & 117 & 1,473 & 2,926 & 2,930 & 2,928 & 2,948 & +0.7 & 2,940 & +0.4 & 1,484 & +0.7 & 34 & 18.6 & 1.5 & 17.1 & $*^{*} \mathrm{P}-\mathbf{3}$ & 10.3 & -0.7 & 11.0 & * $\quad \mathrm{P}-4$ \\
\hline Ocmulgee $\ddagger$ & Jackson, Ga. & $34 \mathrm{C}$ & 114 & 2,801 & 2,808 & 2,808 & 2,808 & 2,700 & -3.8 & 2,708 & -3.7 & 2,787 & -0.5 & 27 & 21.6 & 6.3 & 15.3 & *† $\mathrm{P}-3$ & 10.3 & 1.0 & 9.3 & * SP-4 \\
\hline Rio Chama & Parkview, N. M. & $35 \mathrm{C}$ & 123 & 526 & 2,762 & 2,759 & 2,760 & 2,805 & +1.6 & 2,832 & +2.6 & 532 & +1.1 & 35 & 23.4 & 6.8 & 16.6 & $\mid \begin{array}{lll}* \dagger & P-4\end{array}$ & 17,6 & 6.4 & 11.2 & $\mathrm{P}-1$ \\
\hline Farmington & Rainbow, Conn. & $36 \mathrm{C}$ & 89 & 676 & 2,617 & 2,610 & 2,614 & 2,623 & +0.3 & 2,639 & +1.0 & 676 & 0 & 24 & 15.6 & 6.1 & 9.5 & $\begin{array}{ll}t & \mathrm{P}-2\end{array}$ & 23.8 & 16.3 & 7.5 & $\begin{array}{lll}\dagger & \mathrm{P}-2\end{array}$ \\
\hline Verde & Bartlett Dam, Ariz. & $37 \mathrm{C}$ & 102 & 505 & 2,484 & 2,495 & 2,490 & 2,449 & -1.6 & 2,480 & -0.4 & 504 & $-0,2 \mid$ & 28 & 14,2 & 3.4 & 10.8 & * State & 14.7 & 0.4 & 14.3 & *State \\
\hline Red River of the North & Grand Forks, N. D. & $39 \mathrm{C}$ & 101 & 1,622 & 2,239 & 2,239 & 2,239 & 2,239 & 0 & 2,256 & +0.8 & 1,625 & +0.2 & 27 & 22.5 & 9.0 & 13.5 & t SP-5 & 14.0 & 4.5 & 9.5 & * $\quad \mathrm{P}-3$ \\
\hline Housatonic & Gaylordsville, Conn. & $40 \mathrm{C}$ & 96 & 1,016 & 2,179 & 2,181 & 2,180 & 2,182 & 0 & 2,193 & +0.6 & 1,012 & -0.4 & 25 & 16.8 & 2.1 & 14.7 & $\mid \begin{array}{r}\mid \\
\mid\end{array}$ & 14.2 & 7.8 & 6.4 & $\dagger \mathrm{SP}-4$ \\
\hline Rappahannock & Fredericksburg, Va. & $41 \mathrm{C}$ & 113 & 2,204 & 2,164 & 2,163 & 2,164 & 2,130 & -1.6 & 2,127 & -1.7 & 2,206 & 0 & 29 & 26.5 & 8.3 & 18.2 & $\begin{array}{ll}* & \mathrm{P}-3\end{array}$ & 17.1 & 5.0 & 12.1 & * SP-5 \\
\hline Connecticut & No. Stratford, N. H. & $42 \mathrm{C}$ & 103 & 652 & 2,126 & 2,127 & 2,126 & 12 & +0.8 & 46 & +0.9 & 661 & +1.4 & 28 & 17.3 & 1.0 & 16.3 & $\mid * \dagger P-4$ & 16.0 & 6.2 & 9.8 & $+\mathrm{SP}-4$ \\
\hline Colorado & o, Colo. & $43 \mathrm{C}$ & 110 & 565 & 2,116 & 2,122 & 2,119 & 2,112 & -0.3 & 2,127 & +0.4 & 565 & 0 & 27 & 20.7 & 3.8 & 16.9 & $\mid \begin{array}{ll}* \\
*\end{array}$ & 11.1 & 1.8 & 9.3 & * $\mathrm{P}-4$ \\
\hline Williamson & Chilc & $44 \mathrm{C}$ & 103 & 993 & 2,010 & 2,010 & 2,010 & 1,998 & -0.6 & 2,017 & +0.3 & 989 & -0.4 & 27 & 19.8 & 8.0 & 11.8 & $* \quad \mathrm{P}-2$ & 13.1 & 6.6 & 6.5 & * SP-5 \\
\hline Salt & Roosevelt, Ariz. & $45 \mathrm{C}$ & 103 & 611 & 1,943 & 1,947 & 1,945 & 1,941 & -0.2 & 1,967 & +1.1 & 619 & +1.3 & 28 & 14.7 & 2.1 & 12.6 & *State & 14.2 & 1.2 & 13.0 & *State \\
\hline Clinch & Tazewell, Tenn. & $46 \mathrm{C}$ & 105 & 1,214 & 1,942 & 1,943 & 1,942 & 1,934 & $-0: 4$ & 1,949 & +0.4 & 1,210 & -0.3 & 28 & 15.6 & 3.7 & 11.9 & $*{ }^{+}+\mathrm{P}-3$ & 12.7 & 1.5 & 11.2 & *State \\
\hline Sacandaga & Hope, N. Y. & $47 \mathrm{C}$ & 101 & 692 & 1,708 & 1,705 & 1,706 & 1,697 & -0.5 & 1,705 & 0 & 691 & -0.1 & 29 & 13.6 & 0.6 & 13.0 & $* \begin{array}{lll}* & P-3\end{array}$ & 12.1 & 4.0 & 8.1 & * P-3 \\
\hline Perdido $t$ & Barrineau Park, Fla. & $48 \mathrm{C}$ & 97 & 683 & 1,703 & 1,700 & 1,702 & 1,698 & -0.2 & 1,704 & +0.1 & 681 & -0.3 & 24 & 9.8 & 3.5 & 6.3 & SP-4 & 6.7 & & 4.9 & \\
\hline Current . & Eminence, Mo. & $49 \mathrm{C}$ & 107 & 588 & 1,650 & 1,654 & 1,652 & 1,647 & -0 & 1,655 & +0 & 585 & -0.5 & 29 & & 4.8 & 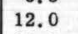 & $P-3$ & $\mid 10.0$ & & 8.0 & \\
\hline Rarite & Bound Brook, N. & $50 \mathrm{C}$ & 88 & 915 & 1,216 & 1,226 & 1,221 & 1,205 & -1.3 & 1,212 & -0.1 & 902 & -1.4 & 26 & 21.0 & 10.0 & 11.0 & P-2 & 8.9 & 1.1 & 7.8 & *† $\mathrm{P}-2$ \\
\hline Choccolocee & Lincoln, Ala. & $51 \mathrm{C}$ & 125 & 882 & 1,221 & 1,221 & 1,221 & 1,200 & $-1.7 \mid$ & 1,205 & -1.3 & 886 & +0.5 & 28 & 18.0 & 5.2 & 12.8 & $P-4$ & 11.4 & 3.4 & 8.0 & $*+$ P -3 \\
\hline Neosho : & Iola, Kans. & $52 \mathrm{C}$ & 108 & 1,069 & 1,165 & 1,166 & 1,166 & 1,141 & $|-2.1|$ & 1,150 & -1.4 & 1,064 & -0.5 & 26 & 13.1 & 2.9 & 10.2 & $* \dagger P-2$ & 12.0 & 3.0 & 9,0 & tHFA \\
\hline Allegheny & Kinzua, Pa. & $53 \mathrm{C}$ & 119 & 1,169 & 1,133 & 1,135 & 1,134 & 1,124 & $|-0.9|$ & 1,133 & 0 & 1,166 & -0.3 & 31 & 20.1 & 2.5 & 17.6 & $* P_{5} 4$ & 9.6 & 3.1 & 6.5 & $*$ P-3 \\
\hline Mulberry & Mulberry, Ark. & $54 \mathrm{C}$ & 76 & 662 & 1,030 & 1,030 & 1,030 & 1,036 & +0.6 & 1,038 & +0.8 & 656 & -0.9 & 26 & 14.3 & 3.9 & 10.4 & $\begin{array}{ll}* & \mathbf{P}-2\end{array}$ & 9.2 & 1.1 & 8.1 & * SP-7 \\
\hline Sevier & Juab, Utah & $55 \mathrm{C}$ & 100 & 455 & 1,002 & 1,003 & 1,002 & 992 & -1.0 & 999 & -0.3 & 452 & -0.7 & 26 & 13.7 & 4.6 & 9.1 & * SP-4 & 7.0 . & 1.1 & 5.9 & * $\mathrm{SP}=4$ \\
\hline Youghiogheny & Confluence, $\mathrm{Pa}$. & $56 \mathrm{C}$ & \begin{tabular}{|c|}
$\mid 27$ \\
127
\end{tabular} & 808 & 999 & 1,000 & 1,000 & 1,026 & +2.6 & 1,031 & +3.1 & 814 & +0.7 & 27 & 19.6 & 3.2 & 16 & * $\quad \mathrm{P}-4$ & 8.9 & 4 & 6.4 & $* \quad \mathrm{P}-3$ \\
\hline Kalamazoo & Battle Creek, Mich. & $57 \mathrm{C}$ & 11 & 58 & 9 & 98 & 986 & 20 & +0.4 & 996 & +1 & 580 & -0.2 & 27 & & 1 & 6 & $\begin{array}{lll}* & P-2\end{array}$ & 13.0 & 3.0 & 10.0 & $+\quad P-2$ \\
\hline Neversink & Oakland Valley, N. Y. & $58 \mathrm{C}$ & 10 & 4 & 9 & 9 & 9 & 9 & $-1,7$ & 927 & -1.6 & 411 & -1.2 & 28 & 2.2 & 0.7 & 11.5 & * $\quad 7-3$ & 8.9 & 2.2 & 6.7 & * $\quad$ P-3 \\
\hline Chippewa & Bruce, Wis. & $59 \mathrm{C}$ & 100 & 5 & 78 & 78 & 7 & 7 & +0.4 & 791 & +0.8 & 541 & -0.7 & 26 & 19.0 & 5.0 & 14.0 & tHFA & 14.7 & 3.4 & 11.3 & tHFA \\
\hline Tuolumme & fetchy, Cali & $60 \mathrm{C}$ & 125 & 5 & 7 & & & & 0 & 774 & 0 & 522 & -0.2 & 36 & 16.5 & 2.0 & 14.5 & *State & 12.7 & 2.2 & 10.5 & *† P-3 \\
\hline So. Chickamauga & Chickamauga, Ten & $61 \mathrm{C}$ & 90 & 4 & 6 & 6 & 6 & 6 & -0.6 & 669 & 0 & 405 & 0 & 25 & & 2.5 & 9.2 & * SP-4 & 9.6 & -2.1 & $\mid 11.7$ & *State \\
\hline & So. Royalston, Mass. & $62 \mathrm{C}$ & 97 & 3 & 6 & $\theta$ & 6 & 642 & +1 & 644 & +1.6 & 322 & +1.3 & 27 & 21.5 & 6.3 & 15.2 & $\begin{array}{ll}t & \mathbf{P}-2\end{array}$ & 7.8 & 1.8 & 6.0 & * P -3 \\
\hline Vermilion & Danville, IIl, & $63 \mathrm{C}$ & 82 & 42 & 507 & 5 & 5 & 502 & -1.2 & 506 & -0.4 & 420 & +1.6 & 25 & 19,2 & 1.3 & 17.9 & * SP-5 & 13.2 & 3.7 & 9.5 & * P-2 \\
\hline Truckee & Reno, Nev. & $64 \mathrm{C}$ & 100 & 29 & 482 & 48 & 482 & 495 & +2.7 & 488 & +1.2 & 291 & -0.3 & 30 & 14.6 & 3.3 & 11.3 & *State & 13.2 & 3.9 & 9.3 & $* \dagger \mathrm{P}-3$ \\
\hline Shoshone & ron, $\mathrm{V}$ & $65 \mathrm{C}$ & 94 & 277 & 465 & 466 & 466 & 457 & -1.9 & 460 & -1.3 & 276 & $-0,4$ & 27 & 12.6 & -1.1 & 13.7 & $* \uparrow$ P-4 & 10.2 & & 8. 1 & $\begin{array}{l}1+1 \\
\end{array}$ \\
\hline Carrabassett & No. Ansc & $66 \mathrm{C}$ & 94 & 389 & 389 & 38 & 3 & 382 & -1.8 & 385 & -1 & 384 & -1.3 & 2 & 1 & & 11.1 & $\left|\begin{array}{ll}1 & -2-1 \\
t & \mathbf{P}-1\end{array}\right|$ & 7.7 & & 7.3 & * $\quad \mathrm{P}-4$ \\
\hline Pearl $\ddagger$ & Edinburg, Miss. & $67 \mathrm{C}$ & 106 & 229 & 294 & 294 & 2 & 292 & $\mid \begin{array}{l}-0.7 \\
-0.7\end{array}$ & 296 & +0.7 & 229 & 0 & 24 & 4.8 & 5.4 & 9.4 & P-1 & 7.3 & 1.2 & 6.1 & * $\quad \mathrm{P}-2$ \\
\hline Rum & St. Francis, M.inn. & $68 \mathrm{C}$ & 106 & 427 & 275 & 275 & 275 & 277 & +0.7 & 279 & +1.4 & 424 & -0.7 & 26 & 16.5 & 6.0 & 10.5 & $P-2$ & 10.0 & 1.5 & 8.5 & † SP-5 \\
\hline Gallatin & Gallatin Gateway, Mont. & $69 \mathrm{C}$ & 93 & 137 & 271 & 271 & 271 & 271 & 0 & 275 & +1.5 & 137 & $\theta$ & 26 & 12.1 & 4.9 & 7.2 & SP -4 & 8.4 & 2.8 & 5.6 & $*$ SP -4 \\
\hline Diamond & Wentworth Location, N. H & $70 \mathrm{C}$ & 83 & 198 & 179 & 179 & 179 & 175 & -2.8 & 174 & -2.8 & 199 & +0.5 & 26 & 17.5 & 8.0 & 9.5 & $P-2$ & 7.2 & 2.7 & 4.5 & it $P-3$ \\
\hline
\end{tabular}

Experienced in mean-section method

*† Experienced in both methods

‡ Boat Measurements 


\section{Special Study for Few Measurements}

The tabulation and computations were checked and examined for errors. The ten normal measurements in which discharge differed 4 percent or more from the true discharge were singled out for further study to determine if possible whether the large error was due to poorly-selected observation stations, of to irregular cross-section and velocity. For three of these measurements, $27 \mathrm{~W}, 38 \mathrm{~W}$, and $42 \mathrm{~W}$, an attempt was made to select stations to improve the accuracy of computed discharge, still keeping within the limitations of number of observations, etc. as previously described. Later for all measurements, observation stations for corresponding new normal measurements were selected generally as the next adjacent stations on the right of those selected for the original normal measurement. A third normal measurement was derived in a similar manner. This method porhaps gare a set, of points in some cases that would not be taken in the field, but it gave an idea of the variation which might occur with different selections of observation stations.

In addition, from each type of measurement one normal measurement that o ompared favorably with the special measurement for accuraoy was selscted for a similar study of the result of varying the combinations of observation stations. This was done to see if the same range in errors sould be produced in those which appeared to be nearly correct in the original selection, as existed in those which were over 4 percent. The same procedure of progressive selection of observation stations was followed. 


\section{ANALYSIS OF RELATIVE ACCURACY}

\section{Average Percent Differences}

The two essential elements to be compared in this study are the relative accuracy of results obtained by the two methods of computation and the time saved in using one method instead of the other. The question of accuracy will be taken up first in this analysis.

The simplest and easiest picture for comparing the accuracy element is the average of the percent differences. The data for percent differences for all measurements shown in Table 1 have been summarized in Table 2. The first half of the table shows the average percent difference without regard

Table 2.-.-Comparison of percent differences from true discharge for types of measurement

\begin{tabular}{|c|c|c|c|c|c|}
\hline \multirow{2}{*}{ Type } & \multirow{2}{*}{$\begin{array}{l}\text { No. of } \\
\text { Meas. in } \\
\text { Group }\end{array}$} & \multicolumn{2}{|c|}{$\begin{array}{l}\text { Average Percent Diff. } \\
\text { Without Regard to Sign }\end{array}$} & \multicolumn{2}{|c|}{$\begin{array}{l}\text { Average Percent Diff. } \\
\text { with Regard to Sign }\end{array}$} \\
\hline & & Mean-Sec. & Mid-Sec. & Mean-Sec. & Mid-Sec。 \\
\hline Bridge & 63 & $1 . \overline{46}$ & 1.22 & -0.97 & -0.37 \\
\hline Wading & 80 & 1.38 & 1.24 & -0.66 & -0.04 \\
\hline Cable & 70 & 0.98 & 0.82 & -0.54 & -0.03 \\
\hline$\overline{\text { Ave。 }}$ & 213 & 1.27 & 1.09 & -0.72 & -0.15 \\
\hline
\end{tabular}

to algebraic sign, and is the sum of the percent difference figures divided by the number of measurements. Those figures show the average of deviations from the true discharge for types of measurements regardless of direction. The second half of the table shows the average percent differenoe taking into 
account the sign. These figures were obtained from the algebraic total of the percent differences divided by the number of measurements, and are more important in what they show than those in the previous half of the table. Although it is necessary to have both averages to get a true comparison, the latter are more important because in the actual use of discharge measurements for rating curves, the direction of the deviation is considered. The rating curve essentially is an average of the measurements taking into account the algebraic sign. The average percent difference without regard to sign for the measurements used in this study is smaller by about 0.2 percent for the mid-section method. Likewise, the average percent differ ence with regard to sign for the mid-section method is about 0.6 percent smalier. It is interesting to note that the results of the cable measurements seem to be the most accurate for the three groups, with wading second, and bridge third. This may be explained in part by. the fact that cableways are usually located at better cross-sections for discharge measurements.

The maximum plus percent differences and maximum negative percont diftexences for a pair of computations in each group are shown in Table 3 . Except for measurement 56C "mean-section", these figures are also the highest individuel percent differences in the groups. Measurement 64C had a percent difference of 2.7 for the "mean-section". It is apparent from a study of this table that there is not much difference in the two methods of computation when for some reason a measurement is off a large amount. Again the cable group shows the smallest range in percent difference. 
Table 3.--Comparison of extremes in percent differences from true discharges for types of measurements

\begin{tabular}{|c|c|c|c|c|c|c|c|c|}
\hline \multirow{2}{*}{ Type } & \multicolumn{3}{|c|}{$\begin{array}{l}\text { Maximum Plus } \\
\text { Percent Difference }\end{array}$} & \multicolumn{3}{|c|}{$\begin{array}{l}\text { Maximum Negative } \\
\text { Percent Difference }\end{array}$} & \multicolumn{2}{|c|}{ Range } \\
\hline & $\begin{array}{l}\text { Meas. } \\
\text { No. }\end{array}$ & $\begin{array}{l}\text { Mean-- } \\
\text { Sec. }\end{array}$ & $\begin{array}{l}\text { Mid- } \\
\text { Sec. }\end{array}$ & $\begin{array}{l}\text { Meas. } \\
\text { No. }\end{array}$ & $\begin{array}{l}\text { Mean- } \\
\text { Sec. }\end{array}$ & $\begin{array}{l}\text { Mid- } \\
\text { Sec。 }\end{array}$ & $\begin{array}{l}\text { Meano } \\
\text { Sec. }\end{array}$ & $\begin{array}{l}\text { Mid- } \\
\text { Sec。 }\end{array}$ \\
\hline Bridge & $31 \mathrm{~B}$ & 3.1 & 3.5 & $38 B$ & 7.4 & 5.7 & 10.5 & 9.2 \\
\hline Wading & $53 w$ & 3.4 & 4.3 & $77 w$ & 9.0 & 9.6 & 12.4 & 13.9 \\
\hline Cable & $56 \mathrm{C}$ & 2.6 & 3.2 & $34 \mathrm{C}$ & 3.8 & 3.7 & 6.4 & 6.8 \\
\hline
\end{tabular}

Graphical Comparison of Percent Differences

In order to show these data graphically, the percent difference of the mid-section was plotted against the percent difference of the mean-section method for each group (see Figs. 2, 3, and 4). A. line was drawn through the plotted points in such a way as to delineate the average of the group. For comparative purposes a dashed line was drawn through the origin and at a slope of unity to indicate a line of equal percent difference. It can bo seen from this line that if a relationship existed between the plotted points such that a line through them had a slope less than one the meansection method would have the higher degree of accuracy. Also, if the slope of the line was greater than one, the mid-section method would have the higher accuracy. The slope of these relationship lines as drawn through the plotted points does not differ greatly from unity, but if anything, it is in tho direction indicating a slightly greater accuracy for the mid-section method as far as a general relationship is concerned. The important thing to note here is the fact that the averago error of these group samples is not zero, as also shown in the comparison of error 
BRIDGE MEASUREMENTS

Comparison of Percent Differences from True Discharge.

Mean-Section Method Computation vs. Mid-Section Method.

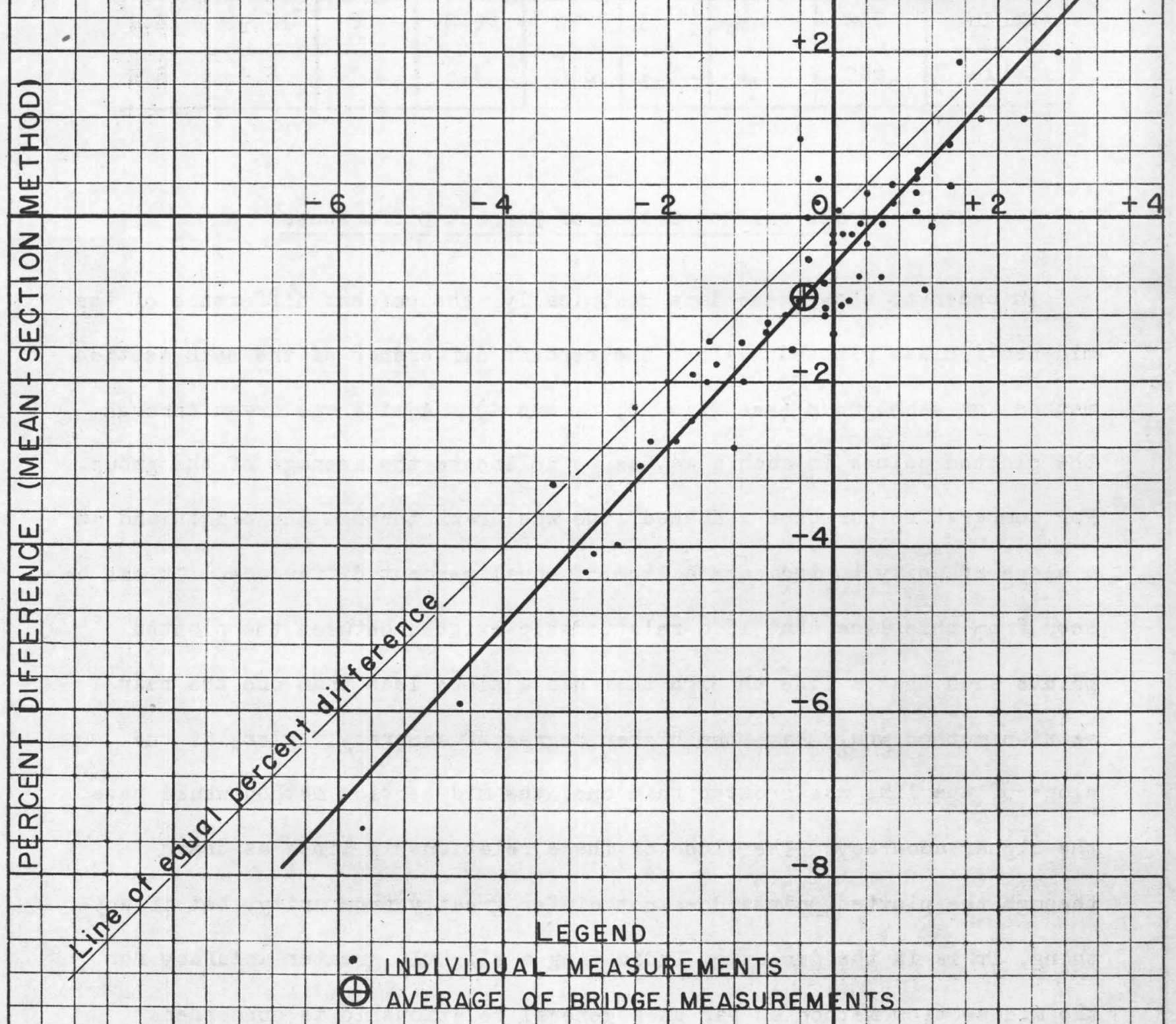

PERCENT DIFFERENCE (MID-SECTION METHOD) FIGURE 2. - Comparison of percent differences for bridge measurements. 
23

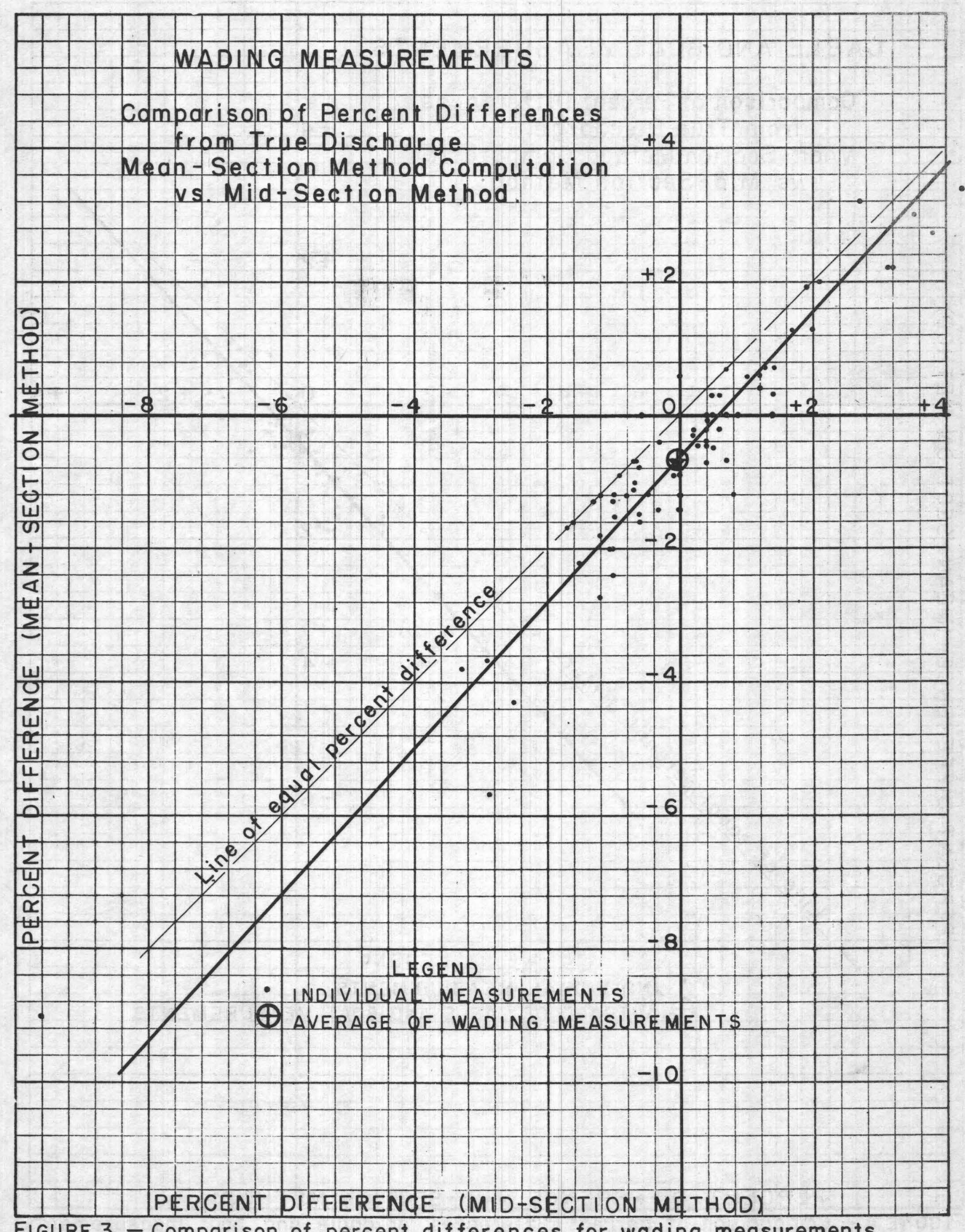

FIGURE 3. - Comparison of percent differences for wading measurements. 
CABLE AND -BOAT MEASUREMENTS

Camparison of Percent Differences from True Discharge.

Mran-Section Method Computation vs. Mid-Section Method.

$+4$

$\begin{array}{r} \\ +2 \\ \hline\end{array}$

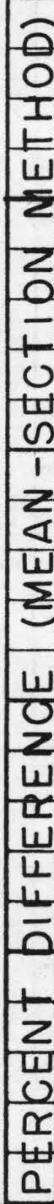

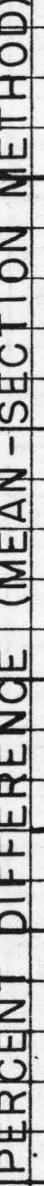

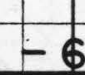

$-4$

$-2$

LEGEND

- individual melasurements

- average of cable and boat measurements 
table, but in the negative quadrant and oloser to zero for the midesection method in all cases. From the distribution of the points on these graphs it is apparent that the results in the majority of the normal measurements used in this sampling are smaller than the assumed true discharge of the specirl measurements. Because of this the average error for the midsection method is smaller and nearer zero than that for the mean-section method.

\section{Statistical Analysis of Data}

In a.11 comparative studies which have to do with intrepreting of experimental data as based on a sample, there are always soocalled experimental exrors involved. To judge the soundness and value of the results from any such work, these results should be compared with an estimato of its error. A statistician calls this process a "test of significance". This test enabies one to decide whether the results are based on adequate ovidence and whether the effects are real and not due to accidental or chance sampling.

Without going too fax into the science of statistics, the first test of significance made with the accuracy element of this study was to detero mine whether or not the difference in the percent error of the mid-section and mean-section mothods is significantly different from zero. Wo might expect that if there is no inherent difference between these methods of computation, the difference in error would average zero. Arithmetically, this average of the percent differences is about minus 0.6 percento To test whether this difference is significantly real and not a result of poor or inadequato sampling, a test known as "student's t" was applied. 
This test showed that the difference in percent error between the two methods was significantly different from zero and that the chances are less than one in one hundred that the apparent difference in results is due to chance. Considering Figs. 2, 3, and 4, we have shown that a line having an average intercept on the Y-axis of minus 0.6 percent agrees with the data significantly better than would a line through the origin.

The second test conducted was to compare the spreads or variabilities of tine mean-section percent differences and the mid-section percent differences. This test shows that there is no significant difference in the variabilities of the two methods, that is, when a measurement is "of $f^{\text {" }}$ by one method of computation it is also "off" by the other method. In other words, for all practical purposes the slope of the relationship lines in Figs. 2,3 , and 4 is not significantly different from unity。

\section{Comparison of Area Variation}

As far as area is concerned there was not as wide a variation in percent difference as there is in discharge when the number of observetions is reduced to a normal measurement. The average percent differense emounted to only about 0.3 percent with the smallest figure being for the cable group (see Table 4). The range in difference for area was considerably less than that for discharge, being about one-half the range which oocurred in the discharge errors. The percent difference for area is the same for both the mean-section and mid-section methods of computation because both methods result in identical figures for area using the same dats. 
Table 4.-Comparison of percent differences in area owing to reduced number of observations

\begin{tabular}{c|c|c|c|c|c}
\hline $\begin{array}{c}\text { Type of } \\
\text { Meas。 }\end{array}$ & $\begin{array}{c}\text { No. of } \\
\text { Meas。 } \\
\text { in Group }\end{array}$ & $\begin{array}{c}\text { Ave.Per。 } \\
\text { Diff。 }\end{array}$ & $\begin{array}{c}\text { Max。 Plus } \\
\text { Percent } \\
\text { Diff。 }\end{array}$ & $\begin{array}{c}\text { Max. Neg。 } \\
\text { Percent } \\
\text { Diff。 }\end{array}$ & Range \\
\hline Bridge & 63 & -.42 & +2.3 & -3.4 & 5.7 \\
\hline Wading & 80 & -.32 & +2.9 & -4.0 & 6.9 \\
\hline Cable & 70 & -.29 & +1.6 & -2.4 & 4.0 \\
\hline Ave. & 2.13 & -.34 & & & 5.5 \\
\hline
\end{tabular}

\section{Effect of Velocity Component in Measurement}

Having the percent difference for area computed for a.ll measurements, it is possiblo to derive a comparable figure for velocity by using thi average percent difference for area (Table 4) and the average percent difference with regard to sign for discharge (Table 2). Using the average for all types of measurements, the velocity component of the meansection method of computation has an indicated percent difference of -.38 percent, and the mid-section, +.19 percent, These figures give an indication of the effect of the velocity component of a measurement, particularly as to the weighting of velocity values with corresponding sub-area values in the two methods. It would appear that for the meanssection method, on the average, the velocity is under-weighted and for the midesection method, overweighted. The mid-section method has a positive indicated percent difference for the velocity component and a negative percent difference for area, while the mean-section has negative percent differences for both velocity and area. Consequently, when combined, the components tend to compensate in the mid-section method, but increase negatively in the meansection. This brief analysis deals with an indefinite part of a discharge 
computation and one whose magnitude has been derived indirectly. The net result which shows the overall comparison of these two methods of computation is shown in Table 2 。

\section{Study of Measurements With Over Four Percent Difference}

There were 10 normal measurements out of a total of 213 in which discharge varied 4 percent or more from the special measurement. As stated previously, these measurements were picked out for further analysis by comparing discharges obtained in using other combinations of observation stations. They are listed in Table 5, showing the special measurement data, results from the original selection for a normal measurement, and subsequent selections. The original selection of observation stations for measurement $27 \mathrm{~W}$ was different for the mean-section than for the mid-section method as previousiy described, the only measurement out of the group of 213 that was treated in this way. As can be seen, the mid-section selection gave much better results. For this analysis, a mean-section method computation was made using data for the original mid-section selections, and a mid-section method computation made using data for the original mean-section selection. Comparing the methods for the same station selections, the midusection gave more accurate results. Then a more or less random selection of observation stations was made by picking out those between the stations a.lready used in the two previous attempts. This choice gave the best results for measurement $27 \mathrm{~W}$ both as to discharge and area, with the mid-section method comparing more favorably with the special measur ement。

Measurement $38 \mathrm{~W}$ gave poor results for discharge in the original 
Table 5.-Analysis of normal measurements off over $4 \%$ in original selection

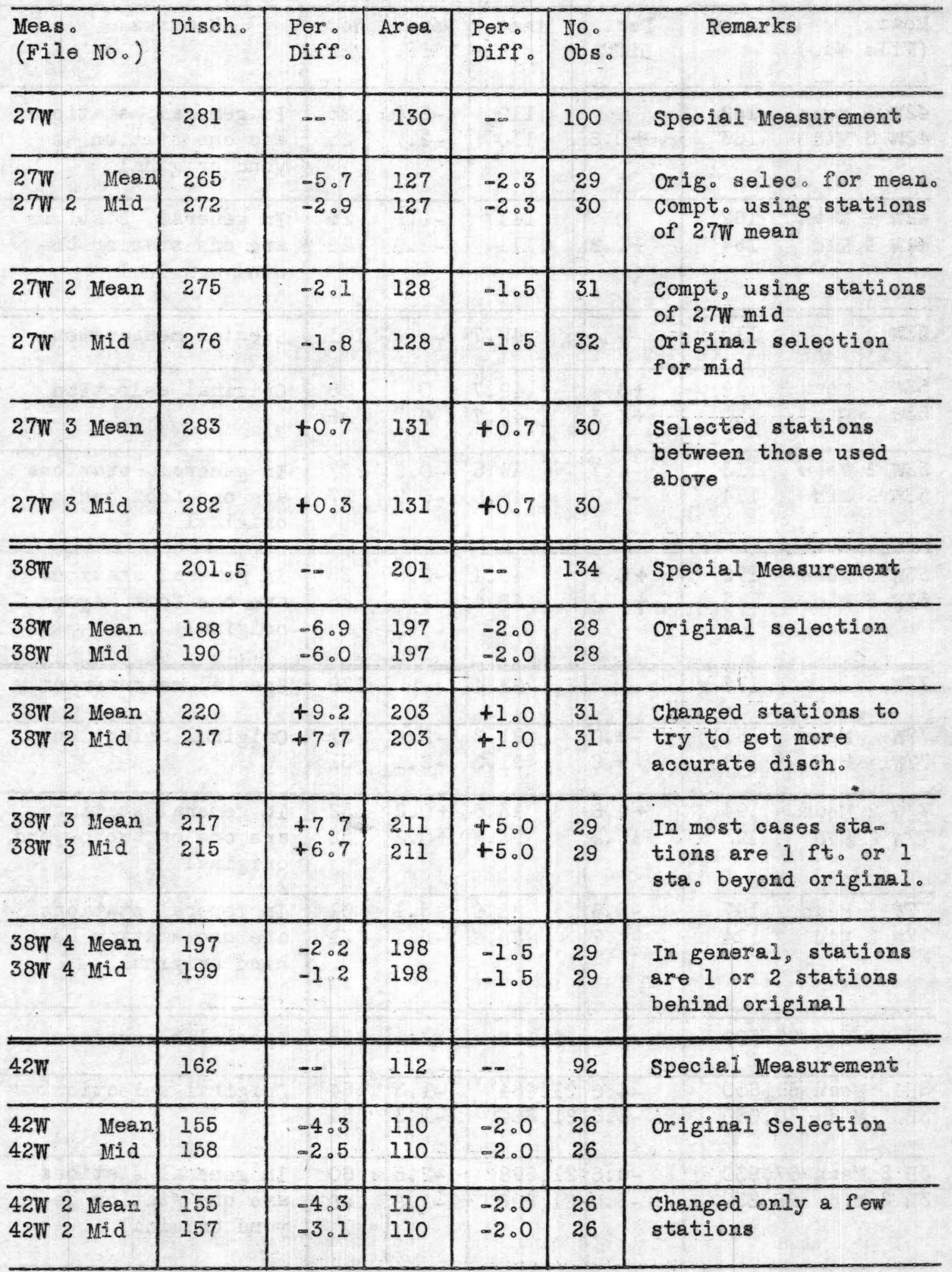


Tabie 5.-Analysis of normal measurements off over $4 \%$ in original selection--Con.

\begin{tabular}{|c|c|c|c|c|c|c|c|}
\hline $\begin{array}{l}\text { Meas. } \\
\text { (File }\end{array}$ & No.) & Disch. & $\begin{array}{l}\text { Per. } \\
\text { Diff. }\end{array}$ & Area & $\begin{array}{l}\text { Per. } \\
\text { Diff. }\end{array}$ & $\begin{array}{l}\text { No. } \\
\text { Obs. }\end{array}$ & Remarks \\
\hline $\begin{array}{l}42 W 3 \\
42 W 3\end{array}$ & $\begin{array}{ll}3 & \text { Mean } \\
3 & \text { Mid }\end{array}$ & $\begin{array}{l}162 \\
163\end{array}$ & $\begin{array}{c}0 \\
+0.6\end{array}$ & $\begin{array}{l}110 \\
110\end{array}$ & $\begin{array}{l}-2.0 \\
-2.0\end{array}$ & $\begin{array}{l}25 \\
25\end{array}$ & $\begin{array}{l}\text { In general, stations } \\
\text { are one station be- } \\
\text { yond original }\end{array}$ \\
\hline $\begin{array}{l}42 W 4 \\
42 W 4\end{array}$ & $\begin{array}{l}\text { Mean } \\
+ \text { Mid }\end{array}$ & $\begin{array}{l}162 \\
164\end{array}$ & $\begin{array}{c}0 \\
+1.2\end{array}$ & $\begin{array}{l}111 \\
111\end{array}$ & $\begin{array}{l}-0.9 \\
-0.9\end{array}$ & $\begin{array}{l}26 \\
26\end{array}$ & $\begin{array}{l}\text { In general, stations } \\
\text { are one station be- } \\
\text { hind original }\end{array}$ \\
\hline $53 w$ & & 115 & $-\infty$ & 49.7 & -- & 101 & Special measurement \\
\hline $\begin{array}{l}53 w \\
53 w\end{array}$ & $\begin{array}{l}\text { Mean } \\
\text { Mid }\end{array}$ & $\begin{array}{l}119 \\
120\end{array}$ & $\begin{array}{l}+3.4 \\
+4.3\end{array}$ & $\begin{array}{l}49.7 \\
49.7\end{array}$ & $\begin{array}{l}0 \\
0\end{array}$ & $\begin{array}{l}28 \\
28\end{array}$ & Original selection \\
\hline $\begin{array}{ll}53 w & 2 \\
53 w & 2\end{array}$ & $\begin{array}{l}\text { Mean } \\
\text { Mid }\end{array}$ & $\begin{array}{l}113 \\
114\end{array}$ & $\begin{array}{l}-1.7 \\
-0.9\end{array}$ & $\begin{array}{l}49 \cdot 6 \\
49 \cdot 6\end{array}$ & $\begin{array}{l}-0.2 \\
-0.2\end{array}$ & $\begin{array}{l}27 \\
27\end{array}$ & $\begin{array}{l}\text { In general, stations } \\
\text { are one foot behind } \\
\text { original }\end{array}$ \\
\hline $\begin{array}{ll}53 W & 3 \\
53 W & 3\end{array}$ & $\begin{array}{l}3 \text { Mesn } \\
3 \text { Mid }\end{array}$ & $\begin{array}{l}116 \\
117\end{array}$ & $\begin{array}{l}+0.9 \\
+1.7\end{array}$ & $\begin{array}{l}48.4 \\
48.4\end{array}$ & $\begin{array}{l}-2.6 \\
-2.6\end{array}$ & $\begin{array}{l}28 \\
28\end{array}$ & $\begin{array}{l}\text { In general stations } \\
\text { are one foot beyond } \\
\text { original }\end{array}$ \\
\hline $77 w$ & & 177 & - & 93.4 & -- & 126 & Specia.l measurement \\
\hline $\begin{array}{l}777 w \\
77 w\end{array}$ & $\begin{array}{l}\text { Mear } \\
\text { Mid }\end{array}$ & $\begin{array}{l}161 \\
160\end{array}$ & $\begin{array}{l}-9.0 \\
-9.6\end{array}$ & $\begin{array}{l}91.2 \\
91.2\end{array}$ & $\begin{array}{l}-2.4 \\
-2.4\end{array}$ & $\begin{array}{l}31 \\
31\end{array}$ & Original selection \\
\hline $\begin{array}{ll}77 w & 2 \\
77 w & 2\end{array}$ & $\begin{array}{l}\text { Mean } \\
\text { Mid }\end{array}$ & $\begin{array}{l}194 \\
195\end{array}$ & $\begin{array}{r}+9.6 \\
+10.2\end{array}$ & $\begin{array}{l}93.6 \\
93.6\end{array}$ & $\begin{array}{l}+0.2 \\
+0.2\end{array}$ & $\begin{array}{l}32 \\
32\end{array}$ & $\begin{array}{l}\text { In general stations } \\
\text { are one or two beyond } \\
\text { original }\end{array}$ \\
\hline $\begin{array}{ll}77 W & 3 \\
77 W & 3\end{array}$ & $\begin{array}{l}3 \text { Mean } \\
3 \text { Mid }\end{array}$ & $\begin{array}{l}167 \\
174\end{array}$ & $\begin{array}{l}-5.6 \\
-1.7\end{array}$ & $\begin{array}{l}88.6 \\
88.6\end{array}$ & $\begin{array}{l}-5.1 \\
-5.1\end{array}$ & $\begin{array}{l}31 \\
31\end{array}$ & $\begin{array}{l}\text { In general stations } \\
\text { are one station be- } \\
\text { hind original }\end{array}$ \\
\hline $3 \mathrm{~B}$ & & 72,590 & -- & 22,146 & -- & 168 & Special measurement \\
\hline $\begin{array}{l}3 \mathrm{~B} \\
3 \mathrm{~B}\end{array}$ & $\begin{array}{l}\text { Mean } \\
\text { Mid }\end{array}$ & $\begin{array}{l}69,650 \\
70,680\end{array}$ & $\begin{array}{l}-4.0 \\
-2.6\end{array}$ & $\begin{array}{l}21,864 \\
21,864\end{array}$ & $\begin{array}{l}-1.3 \\
-1.3\end{array}$ & $\begin{array}{l}54 \\
54\end{array}$ & Original selection \\
\hline $\begin{array}{lll}3 B & 2 & 1 \\
3 B & 2 & 1\end{array}$ & $\begin{array}{l}\text { Mean } \\
\text { Mid }\end{array}$ & $\begin{array}{l}67,830 \\
68,250\end{array}$ & $\begin{array}{l}-6.6 \\
-6.0\end{array}$ & $\begin{array}{l}21,598 \\
21,598\end{array}$ & $\begin{array}{l}-2.5 \\
-2.5\end{array}$ & $\begin{array}{l}60 \\
60\end{array}$ & $\begin{array}{l}\text { In general stations } \\
\text { are one station be- } \\
\text { yond original }\end{array}$ \\
\hline
\end{tabular}


Table 5.- Analysis of normal measurements off over $4 \%$ in original selection-con.

\begin{tabular}{|c|c|c|c|c|c|c|}
\hline $\begin{array}{l}\text { Meas. } \\
\text { (File No.) }\end{array}$ & Disch. & $\begin{array}{l}\text { Pero } \\
\text { Diff. }\end{array}$ & $A x \theta$ & $\begin{array}{l}\text { Per。 } \\
\text { Difí. }\end{array}$ & $\begin{array}{l}\mathrm{No} . \\
\mathrm{Obs} .\end{array}$ & Remar ks \\
\hline $\begin{array}{lll}3 \mathrm{~B} & 3 & \text { Mean } \\
3 \mathrm{~B} & 3 & \text { Mid }\end{array}$ & $\begin{array}{l}70,418 \\
71,668\end{array}$ & $\begin{array}{l}-3.0 \\
-1.3\end{array}$ & $\begin{array}{l}21 ; 750 \\
21 ; 750\end{array}$ & $\begin{array}{l}-1.8 \\
-1.8\end{array}$ & $\begin{array}{l}57 \\
57\end{array}$ & $\begin{array}{l}\text { In general stations } \\
\text { are one station be- } \\
\text { hind original }\end{array}$ \\
\hline $15 \mathrm{~B}$ & 15.483 & $\infty$ & 4,972 & $\infty$ & 120 & Special mea surement \\
\hline $\begin{array}{ll}15 \mathrm{~B} & \text { Mean } \\
15 \mathrm{~B} & \text { Mid }\end{array}$ & $\begin{array}{l}14,848 \\
15,031\end{array}$ & $\begin{array}{l}-4.1 \\
-2.9\end{array}$ & $\begin{array}{l}4,894 \\
4,894\end{array}$ & $\begin{array}{l}-1.6 \\
-1.6\end{array}$ & $\begin{array}{l}46 \\
46\end{array}$ & Original selection \\
\hline $\begin{array}{l}15 \mathrm{~B} \text { \& Mean } \\
15 \mathrm{~B} 2 \mathrm{MId}\end{array}$ & $\begin{array}{l}14,936 \\
15,131\end{array}$ & $\begin{array}{l}-3.5 \\
-2.3\end{array}$ & $\begin{array}{l}4,896 \\
4,896\end{array}$ & $\begin{array}{l}-1.5 \\
-1.5\end{array}$ & $\begin{array}{l}39 \\
39\end{array}$ & $\begin{array}{l}\text { In general stations } \\
\text { are one station beo } \\
\text { yond original }\end{array}$ \\
\hline $\begin{array}{l}15 \mathrm{~B} 3 \mathrm{Mean} \\
15 \mathrm{~B} 3 \mathrm{Mid}\end{array}$ & $\begin{array}{l}15,320 \\
15,460\end{array}$ & $\begin{array}{l}-1.1 \\
-0.1\end{array}$ & $\begin{array}{l}4 ; 990 \\
4,990\end{array}$ & $\begin{array}{l}+0.4 \\
+0.4\end{array}$ & $\begin{array}{l}46 \\
46\end{array}$ & $\begin{array}{l}\text { In general stations } \\
\text { are one station be- } \\
\text { hind original }\end{array}$ \\
\hline $17 \mathrm{~B}$ & 14,982 & $-\infty$ & 4,135 & $-\infty$ & 65 & Special measurement \\
\hline $\begin{array}{l}\text { 17B Mean } \\
17 \mathrm{~B} \text { Mid }\end{array}$ & $\begin{array}{l}14,103 \\
14,313\end{array}$ & $\begin{array}{l}-5.9 \\
-4.5\end{array}$ & $\begin{array}{l}4,032 \\
4,032\end{array}$ & $\begin{array}{l}-2.5 \\
-2.5\end{array}$ & $\begin{array}{l}33 \\
33\end{array}$ & Original selection \\
\hline $\begin{array}{l}17 \mathrm{~B} \text { 2 Msan } \\
17 \mathrm{~B} \text { \& Mid }\end{array}$ & $\begin{array}{l}14,630 \\
14,853\end{array}$ & $\begin{array}{l}-2.3 \\
-0.9\end{array}$ & $\begin{array}{l}4,034 \\
4,034\end{array}$ & $\begin{array}{l}-2.4 \\
-2.4\end{array}$ & $\begin{array}{l}34 \\
34\end{array}$ & $\begin{array}{l}\text { In general stations } \\
\text { are one sta. beyond } \\
\text { original }\end{array}$ \\
\hline $\begin{array}{l}\text { 17B } 3 \text { Mean } \\
\text { 17B } 3 \text { Mid }\end{array}$ & $\begin{array}{l}14,325 \\
14,369\end{array}$ & $\begin{array}{l}-4.4 \\
-4.1\end{array}$ & $\begin{array}{l}4,017 \\
4,017\end{array}$ & $\begin{array}{l}-2.8 \\
-2.8\end{array}$ & $\begin{array}{l}33 \\
33\end{array}$ & $\begin{array}{l}\text { In general station } \\
\text { are one station be- } \\
\text { hind original }\end{array}$ \\
\hline $38 \mathrm{~B}$ & 2,750 & $-\infty$ & 1,115 & $\infty$ & 126 & Special measurement \\
\hline $\begin{array}{ll}\text { 38B } & \text { Mean } \\
\text { 38B } & \text { Mid }\end{array}$ & $\begin{array}{l}2,546 \\
2,594\end{array}$ & $\begin{array}{l}-7.4 \\
-5.7\end{array}$ & $\begin{array}{l}1,100 \\
1,100\end{array}$ & $\begin{array}{l}-1.4 \\
-1.4\end{array}$ & $\begin{array}{l}44 \\
44\end{array}$ & origingl selection \\
\hline $\begin{array}{l}\text { 38B } 2 \text { Mean } \\
\text { 38B } 2 \text { Mid }\end{array}$ & $\begin{array}{l}2,735 \\
2,838\end{array}$ & $\begin{array}{l}-0.5 \\
+3.2\end{array}$ & $\begin{array}{l}1,136 \\
1,136\end{array}$ & $\begin{array}{l}+1.9 \\
+1.9\end{array}$ & $\begin{array}{l}43 \\
43\end{array}$ & $\begin{array}{l}\text { In general tations } \\
\text { are one tation be- } \\
\text { hind original }\end{array}$ \\
\hline $\begin{array}{lll}\text { 38B } & 3 & \text { Mean } \\
\text { 38B } & 3 & \text { Mid }\end{array}$ & $\begin{array}{l}2,671 \\
2,727\end{array}$ & $\begin{array}{l}-2.9 \\
-0.8\end{array}$ & $\begin{array}{l}1 ; 084 \\
1,084\end{array}$ & $\begin{array}{l}-2.8 \\
-2.8\end{array}$ & $\begin{array}{l}46 \\
46\end{array}$ & $\begin{array}{l}\text { In goneral stations } \\
\text { are one station be- } \\
\text { yond or Iglnal }\end{array}$ \\
\hline
\end{tabular}


Table 5.--Analysis of normal measurements off over $4 \%$ in original selection--Con.

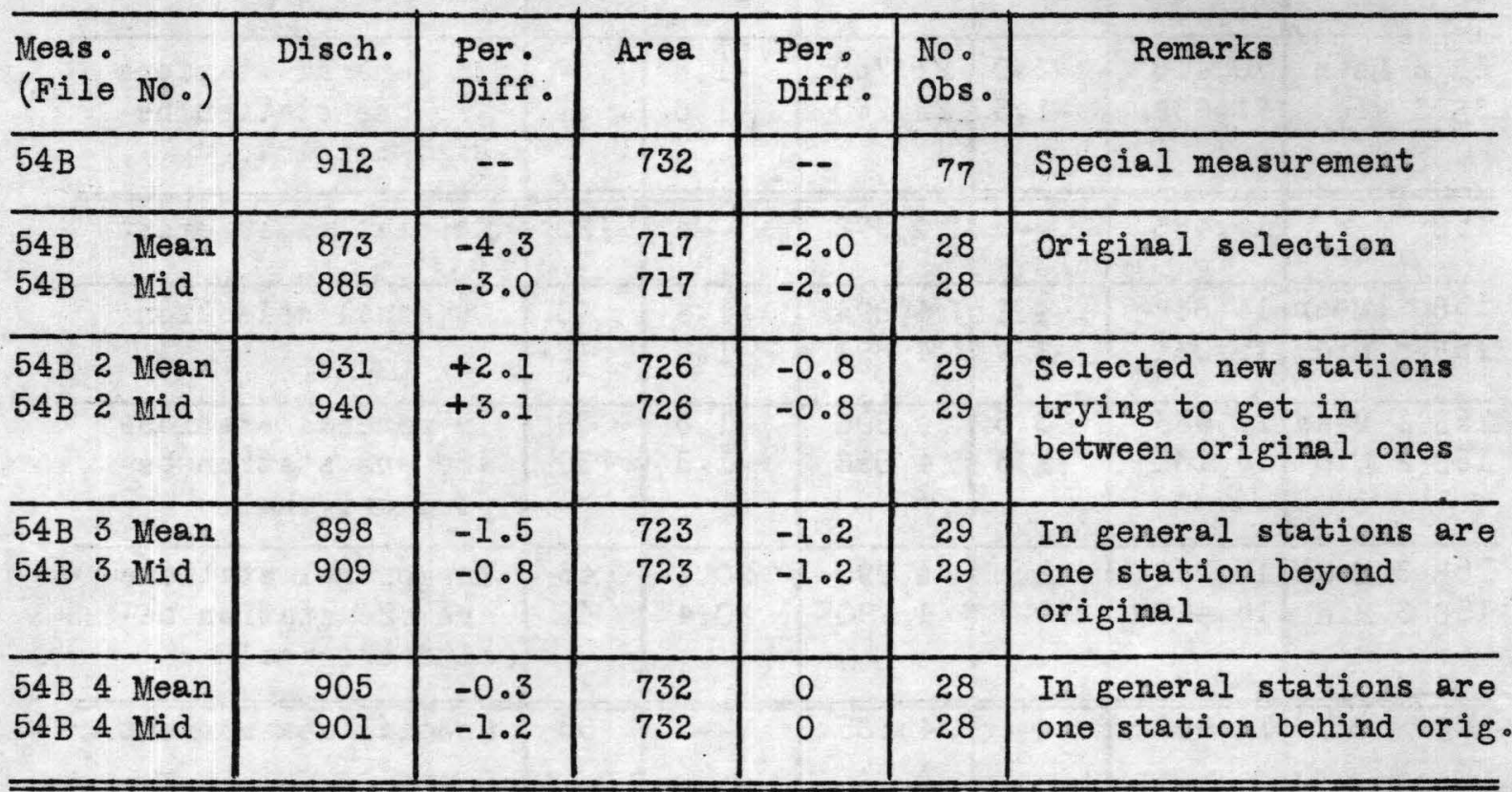

selection, but the area was satisfactory. An attempt was made to change the station selection to get a better discharge result. This resulted in even a worse figure, going from about -6.5 percent in the original selection to +8.5 percent in the second selection. The area, however, was more accurate in the second selections. Following this, two other computations were made using observation stations at other points. It was the latter of these two which produced the most satisfactory result for discharge. In all four combinations of stations, the mid-section method gave better results by an average of about one percent.

only a f'ew of the observation stations in measurement $42 W$ were changed on the second trial. This second selection gave no appreciable difference in either discharge or area. However, two other combinations of observation stations, selected systematically as previously described, gave 
much better discharge agreement without significant change in area, and with the mean-section computation giving more accurate results。

The remaining seven measurements were treated in a similar manner without any special attempt to improve the results. Observation stations were selected at more or less regular intervals behind and ahead of the original selected observation stations. It is noticeable here that the mid-section method gives better results for measurements with computed discharge smaller than the special measurement, and the mean-section method, better for those with computed discharge larger than the special measurement. Since the computed discharges of a majority of the normal measurements are smaller than the special one, the general picture favors the mid-section method. The variation in velocities which might be used, depending upon the selection of observation stations, is shown in Fig. $4 a$ for a discharge measurement with irregular velocity distribution.

\section{Study of Three Measurements \\ Satisfactory in Original Selections}

Measurements $35 \mathrm{~W}, 33 \mathrm{C}$, and $36 \mathrm{~B}$ are normal measurements for which discharge results were very close to those of the special measurements, but were also studied by varying the selection of stations to see if similar results would be obtained as to variations in discharge and area (see Table 5A). There were not as many measurements in this group as in the group with over 4 percent difference due to the lack of personnel and time for computing and checking measurements. As far as these three measurements are concerned there does not seem to be as wide a range in percent difference as appeared in the previous group. The variations and range of percent differd ences for both groups analyzed are shown in Table 6 . 
Table 5A.--Analysis of Normal Measurements Satisfactory in original Selections

\begin{tabular}{|c|c|c|c|c|c|c|}
\hline $\begin{array}{l}\text { Meas. } \\
\text { (File No.) }\end{array}$ & Disch. & $\begin{array}{l}\text { Per. } \\
\text { Diff. }\end{array}$ & Area & $\begin{array}{l}\text { Per. } \\
\text { Diff. }\end{array}$ & $\begin{array}{l}\text { No. } \\
\text { Obs. }\end{array}$ & Remarks \\
\hline $35 W$ & 224 & -- & 105 & -- & 75 & Special measurement \\
\hline $\begin{array}{l}35 w \\
35 w\end{array}$ & $\begin{array}{l}224 \\
224\end{array}$ & $\begin{array}{l}0 \\
0\end{array}$ & $\begin{array}{l}105 \\
105\end{array}$ & $\begin{array}{l}0 \\
0\end{array}$ & $\begin{array}{l}27 \\
27\end{array}$ & Original selection \\
\hline $\begin{array}{l}35 w 2 \text { Mean } \\
35 w 2 \text { Mid }\end{array}$ & $\begin{array}{l}216 \\
216\end{array}$ & $\begin{array}{l}-3.7 \\
-3.7\end{array}$ & $\begin{array}{l}102 \\
102\end{array}$ & $\begin{array}{l}-2.9 \\
-2.9\end{array}$ & $\begin{array}{l}27 \\
27\end{array}$ & $\begin{array}{l}\text { In general stations are } \\
\text { one foot beyond orig. }\end{array}$ \\
\hline $\begin{array}{l}35 w 3 \text { Mean } \\
35 w 3 \text { Mid }\end{array}$ & $\begin{array}{l}231 \\
231\end{array}$ & $\begin{array}{l}+3.1 \\
+3.1\end{array}$ & $\begin{array}{l}107 \\
107\end{array}$ & $\begin{array}{l}+1.9 \\
+1.9\end{array}$ & $\begin{array}{l}27 \\
27\end{array}$ & $\begin{array}{l}\text { In general stations are } \\
\text { one foot behind orig. }\end{array}$ \\
\hline$\overline{33 \mathrm{C}}$ & 2,928 & - & 1,473 & - & 117 & Special measurement \\
\hline $\begin{array}{ll}33 \mathrm{C} & \text { Mean } \\
33 \mathrm{C} & \text { Mid }\end{array}$ & $\begin{array}{l}2,948 \\
2,940\end{array}$ & $\begin{array}{l}+0.7 \\
+0.4\end{array}$ & $\begin{array}{l}1,484 \\
1,484\end{array}$ & $\begin{array}{l}+0.7 \\
+0.7\end{array}$ & $\begin{array}{l}34 \\
34\end{array}$ & Original selection \\
\hline $\begin{array}{lll}33 \mathrm{C} & 2 \text { Mean } \\
33 \mathrm{C} & 2 \mathrm{Mid}\end{array}$ & $\begin{array}{l}2,880 \\
2,886\end{array}$ & $\begin{array}{l}-1.6 \\
-1.4\end{array}$ & $\begin{array}{l}1,440 \\
1,440\end{array}$ & $\begin{array}{l}-2.2 \\
-2.2\end{array}$ & $\begin{array}{l}32 \\
32\end{array}$ & $\begin{array}{l}\text { In general stations are } \\
\text { one station beyond orig. }\end{array}$ \\
\hline $\begin{array}{lll}33 \mathrm{C} & 3 & \text { Mean } \\
33 \mathrm{C} & 3 & \text { Mid }\end{array}$ & $\begin{array}{l}2,947 \\
2,941\end{array}$ & $\begin{array}{l}+0.6 \\
+0.4\end{array}$ & $\begin{array}{l}1,474 \\
1,474\end{array}$ & $\begin{array}{l}+0.1 \\
+0.1\end{array}$ & $\begin{array}{l}34 \\
34\end{array}$ & $\begin{array}{l}\text { In general stations are } \\
\text { one station behind orig. }\end{array}$ \\
\hline $36 \mathrm{~B}$ & 2,889 & - & 1,553 & - & 131. & Special measurement \\
\hline $\begin{array}{ll}36 \mathrm{~B} & \text { Mean } \\
\text { 36B } & \text { Mid }\end{array}$ & $\begin{array}{l}2,896 \\
2,883\end{array}$ & $\begin{array}{l}+0.2 \\
-0.2\end{array}$ & $\begin{array}{l}1,559 \\
1,559\end{array}$ & $\begin{array}{l}+0.4 \\
+0.4\end{array}$ & $\begin{array}{l}42 \\
42\end{array}$ & Original selection \\
\hline $\begin{array}{lll}36 \mathrm{~B} & 2 & \text { Mean } \\
36 \mathrm{~B} & 2 \mathrm{Mid}\end{array}$ & $\begin{array}{l}2,797 \\
2,841\end{array}$ & $\begin{array}{l}-3.2 \\
-1.7\end{array}$ & $\begin{array}{l}1,504 \\
1,504\end{array}$ & $\begin{array}{l}-3.1 \\
-3.1\end{array}$ & $\begin{array}{l}40 \\
40\end{array}$ & $\begin{array}{l}\text { In general tation are } \\
\text { one tation beyond orig. }\end{array}$ \\
\hline 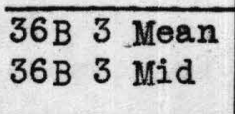 & $\begin{array}{l}2,733 \\
2,781\end{array}$ & $\begin{array}{l}-5.4 \\
-3.7\end{array}$ & $\begin{array}{l}1,478 \\
1,478\end{array}$ & $\begin{array}{l}-4 \cdot 8 \\
-4.8\end{array}$ & $\begin{array}{l}41 \\
41\end{array}$ & $\begin{array}{l}\text { In general stations are } \\
\text { one station behind orig. }\end{array}$ \\
\hline
\end{tabular}


Table 6.-- Variation in Percent Differences from True Discharge (Due to various combinations of observation stations).

\begin{tabular}{|c|c|c|c|c|c|c|c|c|c|}
\hline \multirow[t]{2}{*}{$\begin{array}{c}\text { Meas . } \\
\text { No. }\end{array}$} & \multicolumn{3}{|c|}{$\begin{array}{l}\text { Discharge } \\
\text { Mean-Section }\end{array}$} & \multicolumn{3}{|c|}{$\begin{array}{l}\text { Discharge } \\
\text { Mid-Section }\end{array}$} & \multicolumn{3}{|c|}{ Area } \\
\hline & $\begin{array}{l}\text { Max. } \\
\text { Disch. } \\
\text { \% Diff. }\end{array}$ & $\begin{array}{l}\text { Min. } \\
\text { Disch. } \\
\text { \% Diff. }\end{array}$ & $\begin{array}{l}\text { Range } \\
\text { In } \%\end{array}$ & $\begin{array}{l}\text { Max. } \\
\text { Disch. } \\
\text { \% Diff. }\end{array}$ & \begin{tabular}{|l|} 
Min. \\
Disch. \\
\% Diff.
\end{tabular} & $\begin{array}{l}\text { Range } \\
\text { In } \%\end{array}$ & $\begin{array}{l}\text { Max. } \\
\text { Area } \\
\text { \%Diff }\end{array}$ & $\begin{array}{l}\text { Min. } \\
\text { Area } \\
\text { \%Diff. }\end{array}$ & $\begin{array}{l}\text { Range } \\
\text { In \% }\end{array}$ \\
\hline \multicolumn{4}{|c|}{ Measurements off 4} & \multicolumn{3}{|c|}{ or in original selection } & \multirow{2}{*}{$1+0.7$} & & \multirow[b]{2}{*}{3.0} \\
\hline $27 w$ & $|+0.7|$ & -5.7 & 6.41 & $1+0.3$ & -2.9 & 3.2 & & -2.3 & \\
\hline $38 w$ & $+9 \cdot 2$ & -6.9 & 16.1 & +7.7 & -6.9 & 14.6 & +5.0 & -2.0 & 7.0 \\
\hline $42 W$ & 0 & -4.3 & 4.3 & +1.2 & -3.1 & 4.3 & -0.9 & -2.0 & 1.1 \\
\hline $53 w$ & +3.4 & -1.7 & 5.1 & +4.3 & -0.9 & 5.2 & 0 & -2.6 & 2.6 \\
\hline $77 W$ & +9.6 & $-9 \cdot 0$ & 18.6 & +10.2 & -9.6 & 19.8 & +0.2 & -5.1 & 5.3 \\
\hline $3 B$ & -1.6 & -6.6 & 5.0 & -1.3 & -6.0 & 4.7 & -1.3 & -2.5 & 1.2 \\
\hline $15 B$ & -1.1 & -4.1 & 3.0 & -0.1 & -2.9 & 2.8 & +0.4 & -1.6 & 2.0 \\
\hline $17 \mathrm{~B}$ & -2.3 & -5.9 & 3.6 & -0.9 & -4.5 & 3.6 & -2.4 & -2.8 & 0.4 \\
\hline $38 B$ & +3.2 & -7.4 & 10.6 & -0.5 & -5.7 & 5.2 & +1.9 & -2.8 & 4.7 \\
\hline $54 B$ & +2.1 & -4.3 & 6.4 & +3.1 & -3.0 & 6.1 & 0 & $-2 \cdot 0$ & 2.0 \\
\hline Ave. & & & 11.3 & & & 9.9 & & & 4.2 \\
\hline \multicolumn{4}{|c|}{ Measurements } & \multicolumn{3}{|c|}{ in original selection } & & & \\
\hline $35 w$ & +3.1 & -3.7 & 6.8 & +3.1 & -3.7 & 6.8 & +1.9 & -2.9 & 4.8 \\
\hline$\overline{33 C}$ & +0.7 & -1.6 & 2.3 & +0.4 & -1.4 & 1.8 & +0.7 & $-2 \cdot 2$ & .2 .9 \\
\hline $36 \mathrm{~B}$ & +0.2 & -5.4 & 5.6 & -0.2 & -3.7 & 3.5 & +0.4 & -4.8 & 5.2 \\
\hline Ave. & & & 4.9 & & & 4.0 & & & 4.3 \\
\hline
\end{tabular}




\section{Average Time Savings}

The second element to be considered in this comparative study is the matter of time savings. Because the mid-section method of computation has two less arithmetical processes, averaging of velocities and depths, it is evident that ordinarily this method will consume less time. The extent of this time saving is shown in Table 7 for each group of computers and checkers; that is, those experienced in the mean-section only, those experienced in mid-section only, and those experienced in both methods. The average saving in minutes for each group and for the total has been computed. This study shows that about five minutes would be saved in computing each measurement on the average by using the midsection method, and about three minutes in checking each measurement.

Table 7.--Time Savings in Minutes, Mid-Section Over Mean-Section For Same Measurement

\begin{tabular}{|c|c|c|c|c|c|c|c|c|}
\hline & \multicolumn{2}{|c|}{$\begin{array}{c}\text { Personnel } \\
\text { Experienced } \\
\text { in Mean- } \\
\text { Section } \\
\text { only }\end{array}$} & \multicolumn{2}{|c|}{$\begin{array}{l}\text { Personnel } \\
\text { Experienced } \\
\text { in Mid- } \\
\text { Section } \\
\text { only }\end{array}$} & \multicolumn{2}{|c|}{$\begin{array}{l}\text { Personnel } \\
\text { Experienced } \\
\text { in Both } \\
\text { Methods }\end{array}$} & \multicolumn{2}{|c|}{$\begin{array}{c}\text { All } \\
\text { Personnel }\end{array}$} \\
\hline & Comp。 & $\mathrm{Ch}^{\mathrm{g}} \mathrm{k}$ 。 & Comp。 & $\mathrm{Ch}^{\circ \mathrm{k}}$ 。 & Comp。 & $\mathrm{Ch}^{\circ} \mathrm{k}$. & Comp. & $\mathrm{ch}^{\mathrm{g}} \mathrm{k}$. \\
\hline $\begin{array}{l}\text { Total saving } \\
\text { in Minutes }\end{array}$ & 587.4 & 315.8 & 332.6 & 207.5 & 172.9 & 109.7 & 1092.9 & 633.0 \\
\hline $\begin{array}{l}\text { No. of Meas- } \\
\text { urements }\end{array}$ & 126 & 140 & 45 & 37 & 42 & 36 & 213 & 213 \\
\hline $\begin{array}{l}\text { Ave. saving } \\
\text { per measure- } \\
\text { ment (min.) }\end{array}$ & 4.7 & 2.3 & 7.4 & 5.6 & 4.1 & 3.0 & 5.1 & 3.0 \\
\hline
\end{tabular}




\section{Graphical Comparison of Time Consumption}

In order to show the comparison of time consumed in the computation and checking operations performed in these 213 normal measurements, the time for the mid-section method was plotted against the time for the meansection method for each experience group (Figs.5, 6, 7, 8, 9, 10). The slope of the line drawn from the origin through the average of those points is andication of the relative time consumption as far as the specific operations included in the tests are concerned. It does not show the relation for the computation of a complete measurement as a constant time for the remaining operations common to both methods added to each side will reduce this ratio somewhat. A dashed line at slope of unity shows equal time for each method. It would be expected that all the points would fall to the left of the line of equal time consumption but due to some particular circumstance the mid-section method took longer to compute in a few cases and the points fall to the right of this line. This is more noticeable in the experienced-in-mean group, where apparently the lack of experience in the mid-section method caused more time consumption in that method in a few cases。

The slopes of these lines varied between groups, as might be expected. That for the experienced-in-mid group was the highest since inexperience in the mean-section method would raise the time for that method and increase the difference between the two methods. The slope for experienced-in-mean group was the lowest due to inexperience in the mid-section method increas. ing the time consumption for that method and decreasing the difference between the two methods. The slope for experienced-in-both groups was between the previous two as would be expected. 
COMPUTING MEASUREMENTS

EXPERIENCED - IN - MEAN GROUP

40

comparison of Time Consumption for Methods of Computation.

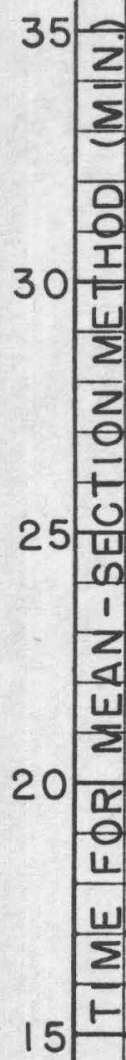
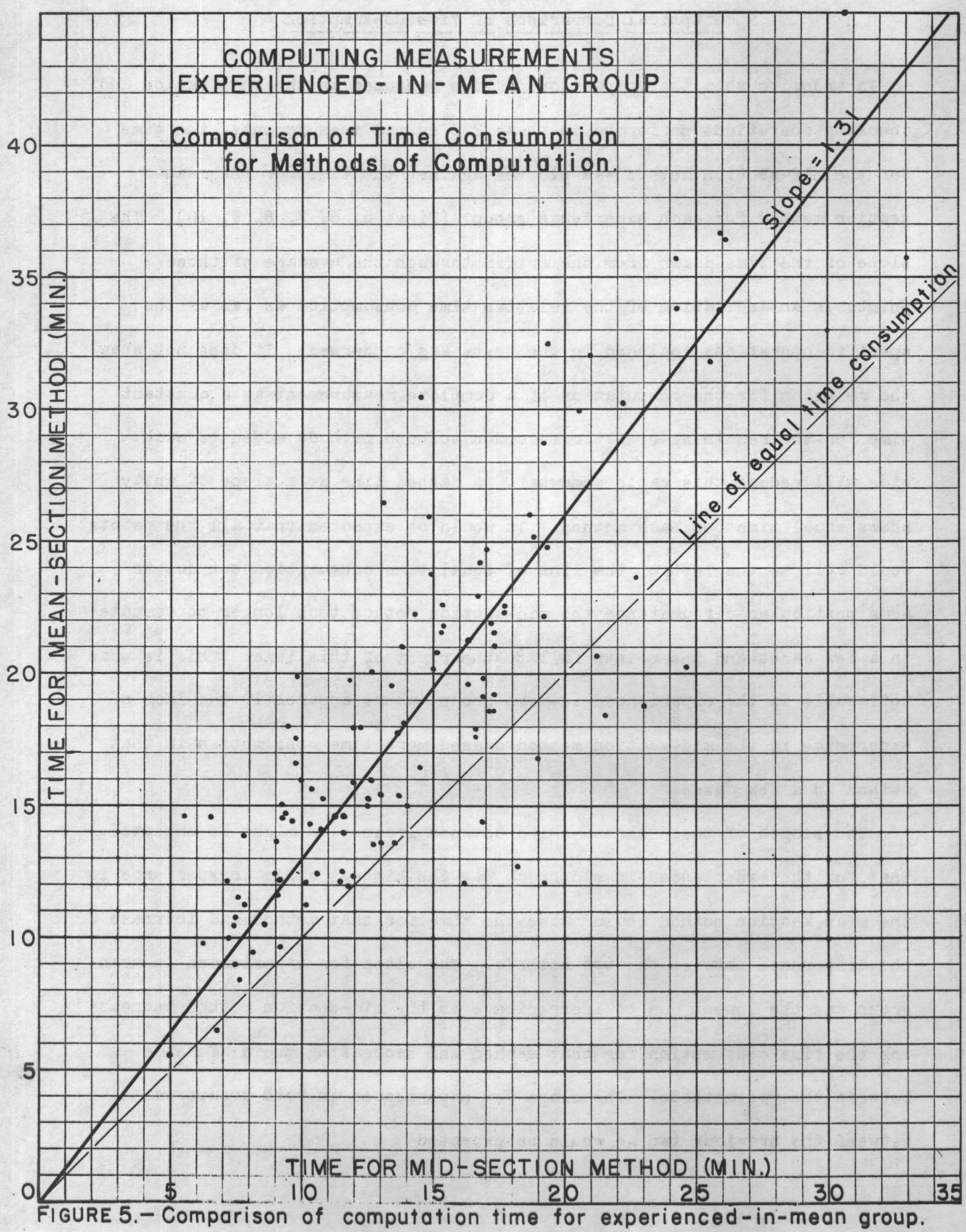
COMPUTING MEASUREMENTS

EXPERIENCED - IN-MID GROUP

Comparison of Time Consumption for Methods of Computalion

35

40 
40

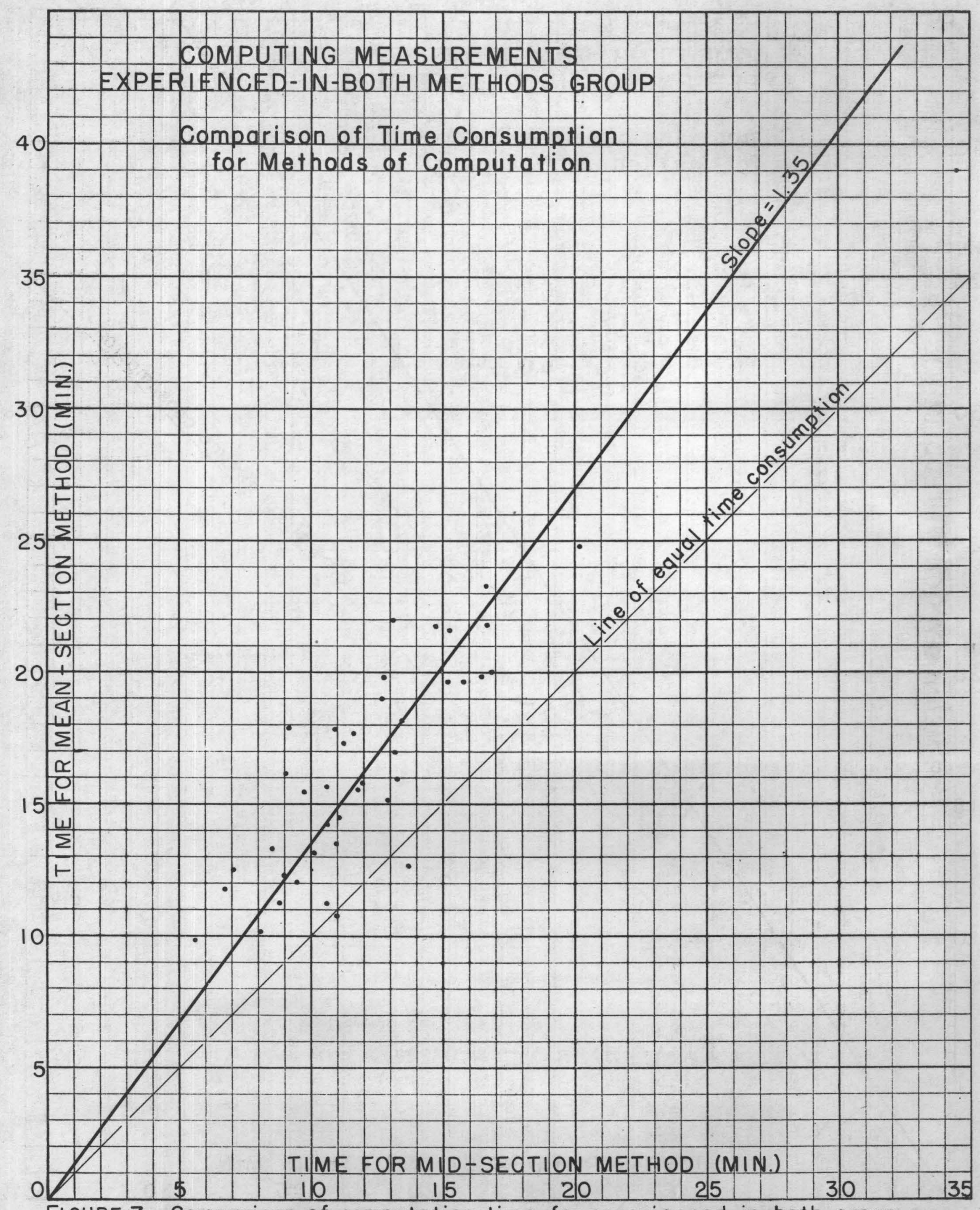

FIGURE 7.- Comparison of computation time for experienced-in-both group. 
CHECKING MEASUREMENTS

EXPERIENCED - IN - MEAN GROUP

comparison of Time Consumption for Methods of Computation

35

30

2

O

i

25

-

\begin{tabular}{|l|}
\hline$z$ \\
\hline 0 \\
\hline 1 \\
0 \\
\hline$\omega$ \\
\hline ज! \\
\hline
\end{tabular}

20

这

15

อ

(1)

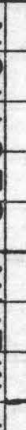

$\sum$

10
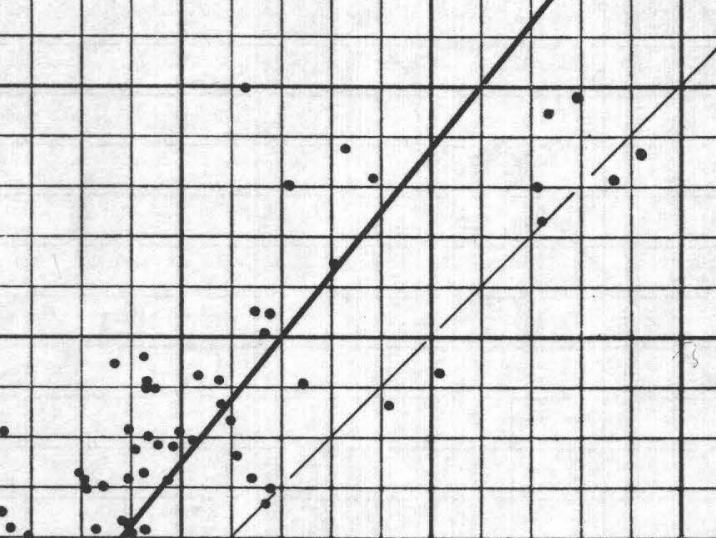
42

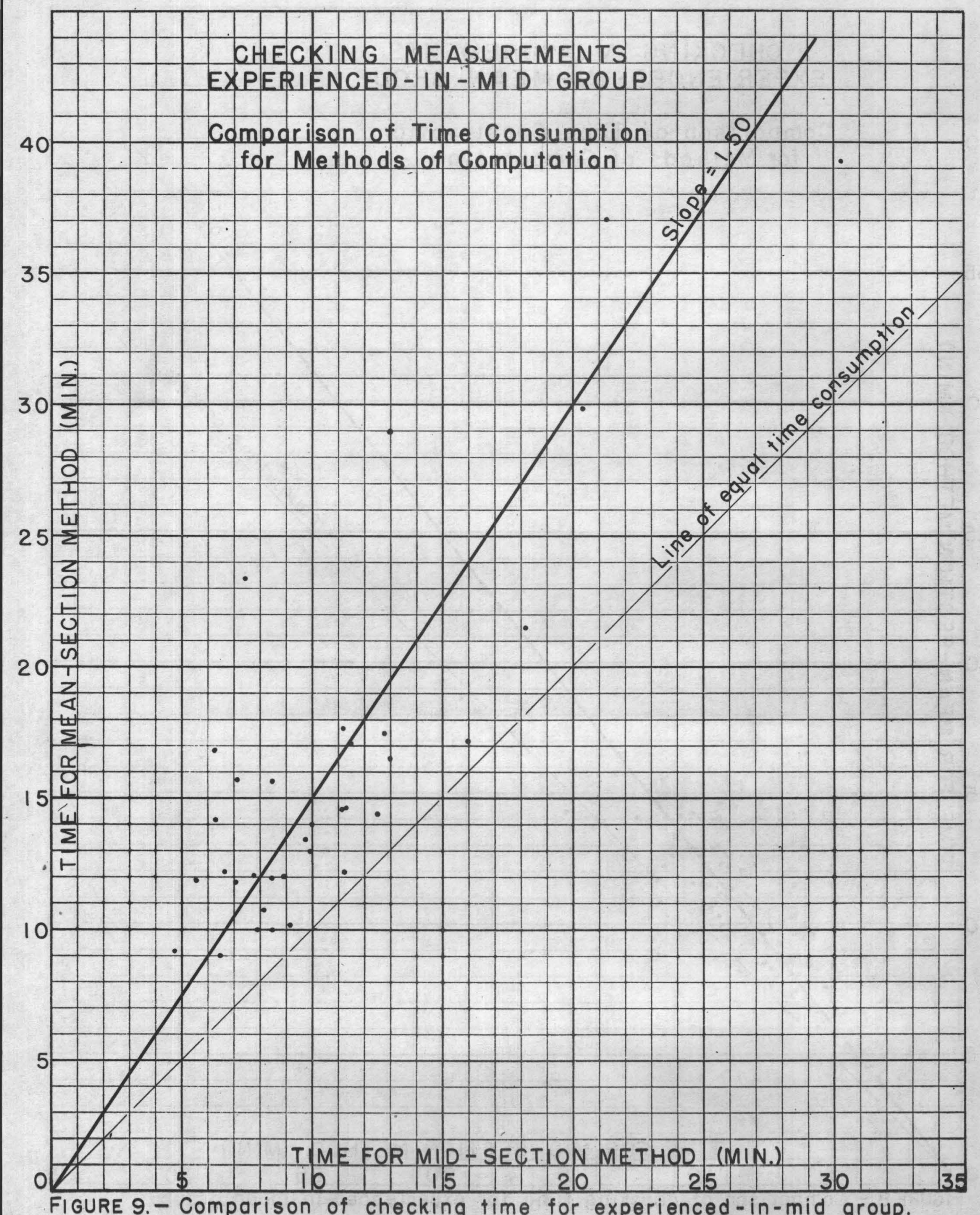


CHECKING MEASUREMENTS

EXPERIENCED-IN-BOTH METHODS GROUP

40 Comparison of Time Consumption for Methods of Computation

35

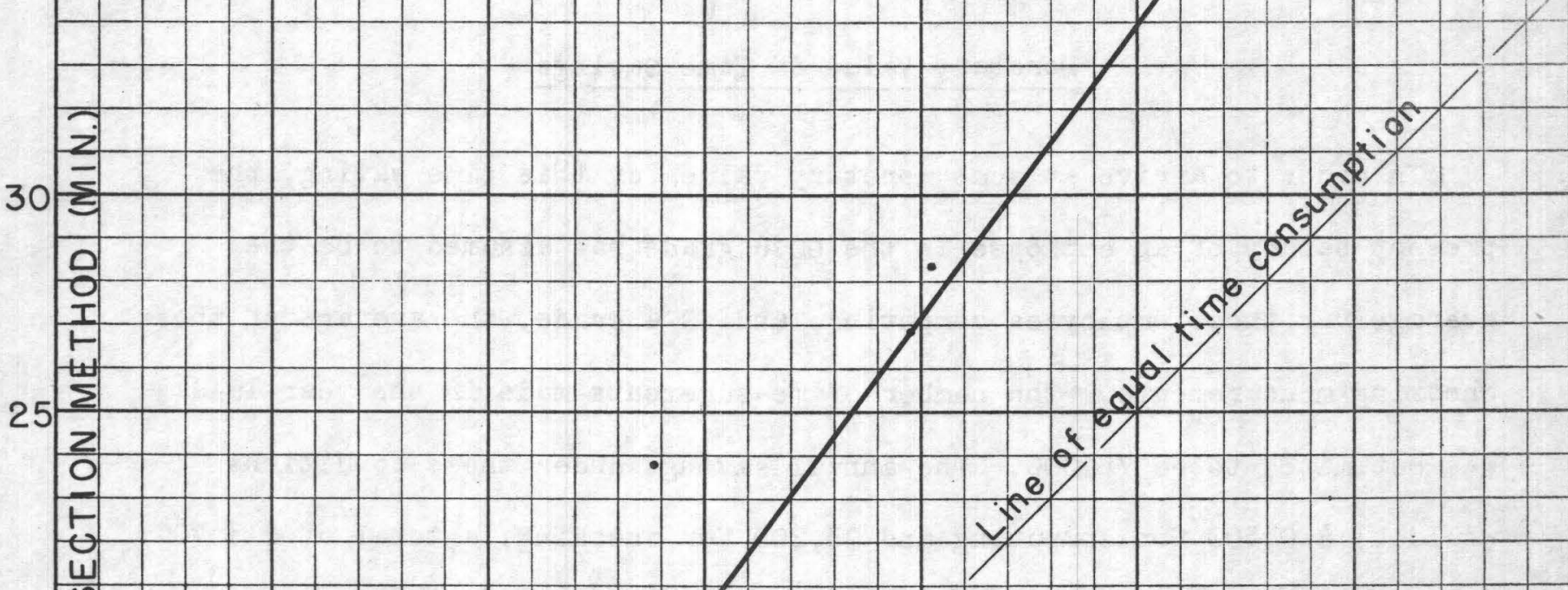

20

7

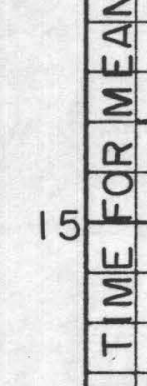

10

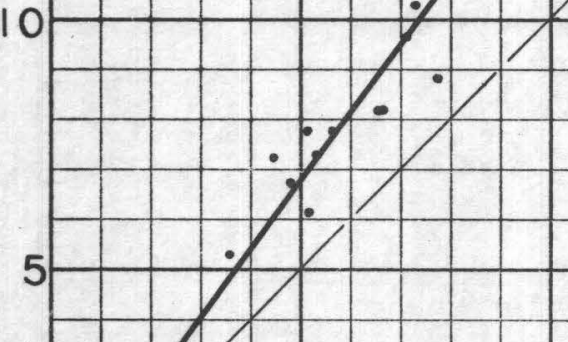

TIME FOR MID-SECTION METHOD (MIN.)

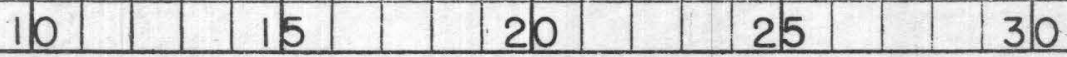

FIGURE 10.-Comparison of checking time for experienced-in-both group. 
The relation between time savings and number of observations per measurement is shown in Fig. Il for those experienced in both methods. Although the points scatter considerably, a line through the average of these points gives a general idea as to the time savings which might be expected for varicus discharge measurements if the number of observations in the mersurements is known.

\section{Monetary Value of Time Savings}

In order to arrive at some monetary value for this time saving, the present salary of an employee in the GS 6 grade was assumed to be the average for those employees computing, and GS 4 grade, the average of those checking measurements. The number of measurements made in the year 1949 was estinated to be 75,000 . The annual savings under these conditions would ba $\$ 10,500$ for computing and $\$ 5,200$ for checking, a total of $\$ 15,700$. However, in the Geological Survey a large share of discharge notes are computed during the process of measurement, and many evening hours are devoted during field trips to completing computations of discharge. It is thought, therefore, that the time sevings for computation should be reduced by about half, and the total savings to the Government might be expected to be in the range from $\$ 10,000$ to $\$ 12,000$ per year if the mid-section method of computation is adopted officially. 
EXPERIENCED IN BOTH METHODS

COMPUTING

CHECKING

70

70

60

60

50

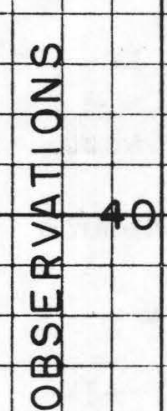

430

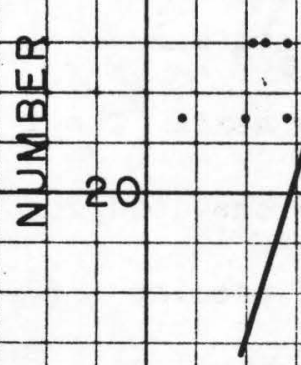

10

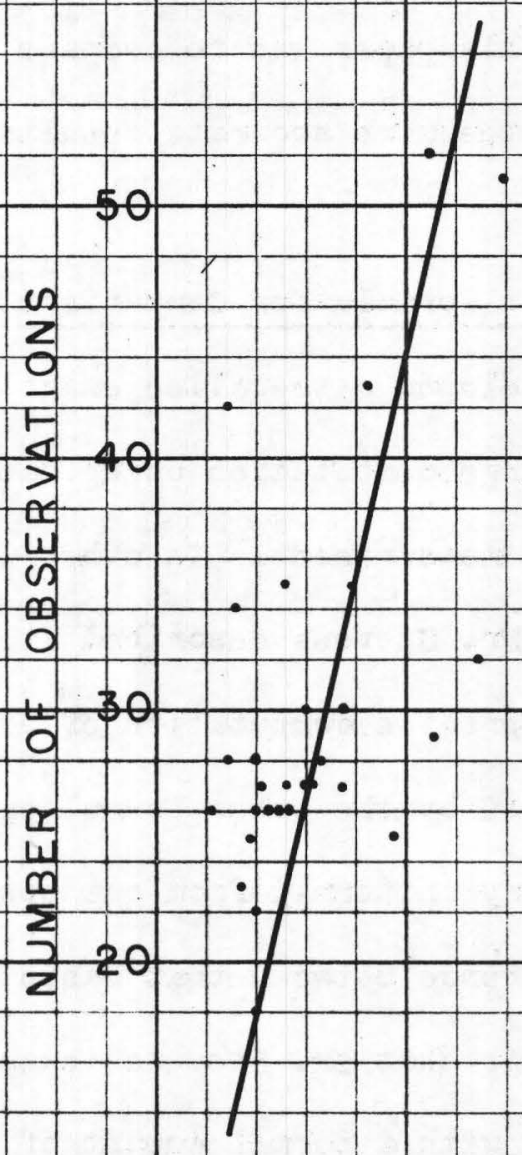

10

TIME SAYINGS (MIN.)

TIME SAVINGS (MIN.)

FIGURE 11.- Relation of time squings to number of observations for computation bv mid-section method vs. mean-section method. 


\section{DISCUSSION OF PREVIOUS INVESTIGATIONS}

\section{Paper by J. C. Stevens}

In many of the discussions on the methods of computation of discharge meaurements, references have been made to a paper prepared in 1908 by J. C. Stevens, then District Engineer of the Portland, Oregon, office. This paper was read at a district engineers ' conference in Washington, D. C., in June 1908, and later published in the June 25, 1908, issue of the Engineering News. Mr. Stevens stated that there were six formulas being generally used then for the computation of measurements. The purpose of his paper was to compare those various formulas to determine which gave the more accurate results.

Use of Exact Formula for Comparison.--For a basis of comparison, Mr. Stevens developed a so-called exact formula which would give the accurate discharge computation using data collected in the usual procedure of a normal measurement. In other words, this exact, or standard formula as Mr. Stevens described it, is simply the prismoidal formula and a more precise computation of discharge using the same data as would be used by the other formulas, assuming that the depth and velocity vary uniformly from one observation point to the next. The chief difference between that basis of comparison and the one used in this study is that Mr. Stevens" exact formula gave a more precise computation with a normal amount of data, whereas, the accurate discharge used in this study was obtained by what the committee felt to be a more precise measurement of the discharge. In arriving at the 
exact formula the author makes the assumption that the depth and velocity vary uniformly from one depth to the next, and from one velocity to the next. This is a logical assumption and is also present in other formulas, but it is a condition that does not always actually exist in the characteristics of rivers and streams. Consequently, even the exact formula may not give the true discharge even though it is more precise as far as making the best use of the observed data is concerned. One way to minimize the effects of this assumption and to take into account varying conditions in depth and velocity is to increase the number of observations of these depths and velocities to be used in the computation of the discharge. For instance, if we assume that the depth and velocity vary uniformly, between two points 10 feet apart, but that it is obvious from inspection that they do not, it is quite logical that more observations taken between these points will measure these variations and make them usable in the computations, thus resulting in a more accurate discharge determination. In other words, as we decrease the distance between the observations of depth and velocity, the higher the probability that these quantities will vary uniformly from one to the next, or that the assumption is true. 
Comparison of Formulas.-- In comparing the mean-section and mid-section formulas with his exact one, $\mathbb{M r}$. Stevens showed that his formula $D$, or what is now called the mid-section method, and formula $B$, the mean-section method, gave consistently the smallest errors, that of $D$ being twice and usually of opposite sign than that of $B_{0}$ which in general is negative. He also proved that this relation was due to the difference in the formulas for those two methods, so that it is evident that under normal conditions the mid-section method will give an equal or higher discharge figure than the mean-section method. This was found to be confirmed in this study also, as was shown in the graphs of Figs. 2,3 , and 4. If the true discharge is in general higher than that computed by either method in a normal set of observations, then it is evident that the mid-section computation will give more accurate results most of the time。

Mr. Stevens made the following statements in his conclusions regarding the relative merits of his formulas $B$ and $D$, or the mean-section and midsection methods.

"(8) The extreme simplicity of Formula D recommends it for general use............. It is well adapted to regular or irregular intervals between points of observation."

"(9) Formula B has the least error of any under conconsideration, but requires two more columns in the notes than the use of $D_{0}$ "

The discussion of Mr. Stevens' paper is presented in this report to bring out the differences between his comparisons and the ones of this study, and also to point out that even forty years ago an attempt was made to settle this question of methods of computation. The object of 
his study was the examination of several formulas for computing stream discharge, with a view to the adoption of one as a standard which will give reliable results under all conditions" At the beginning of his paper. Mx. Stevens also stated that under certain conditions all the formulas considered will give practically the same results, in which case the one involvo Ing a minimum amount of labor should by all means be adopted, and the more so when it is shown that the simplest has the least error under all conditions It would seem that from statements made by Mr. Stevens, his conclusions seemed to be in favor of formula. $D_{2}$ or the mid-section method.

\section{Discussion by C.E。Grunsky}

In the Transactions of the American Society of Civil Engineers, March 1910 , is published a paper by John C. Hoyt on "The Use and Care of the Current Meter, as Practiced by the United States Geological Survey." One of the engineers who presented a discussion of Mr。 Hoyt's paper, Mr. C. E. Grunsky, consulting engineer, brought out the matter of discharge measurement computations. He expressed his preference for a computation method which is now called the mid-section method, and on the basis of his assumptions indicated that it was more accurate than the mean-section method then in use by the Geological Survey. His basis of comparison was a discharge determined by developing a discharge curve by plotting the products of depth and velocity at each observation station as ordinates and connecting the plotted points by a curved line.

\section{Discussions in Water Resources Bulletin}

At various times there have been discussions on oomputation methods published in the Water Resouroos Bulletin, an administrative memorandum 
issued quarterly in the Water Resources Division, Geological Survey. Several engineers of the Water Resources Division have expressed their ideas and opinions on this subject. The following are references to these discussions found in Water Resources Bulletins: Slack, 1224, p. 20; Slack, 325, p. 23; Dalrymple, 542, p. 74; Colby, 842, p. 114; Veatch, 1142, p. 164; Gambrell, 243, p. 14; Colby, 543, p. 63; Eagle, 543, p. 65; Twichell, 543, p. 68; Lord, 1144, p. 180; Pierce, 245, p. 9 .

The above discussions by Mr. Stevens, Mr. Grunsky, and others would be of interest to anyone desiring to do any reviewing on this subject.

\section{CONCLUSIONS}

After viewing the comparisons made in this study, there is no large difference as far as accuracy is concerned. As has been pointed out, the mid-section method gives slightly more accurate results when compared to the true or integrated discharge. Assuming that the true discharge is one obtained by taking a large number of observations, results from the mid-section method, on the average, do not vary as much from this true figure as those from the mean-section method when the observations are reduced to a normal number.

As a matter of emphasis it should be pointed out that this study deals with the accuracy of discharge measurements only in relation to the method of computation. Investigations and experiments have shown that current meters give an accurate determination of the mean velocity by observations taken at the .2 and .8 depths, or .6 depths. It must be assumed that proper care was taken in the measurements of 
water depths and velocities. In other words, this accuracy study is coneerned chiefly with the computations of the discharge measurement, and not with that portion of making the measurement which involves equipment and personal influences.

The measurements used in this study were made under a wide variety of field conditions covering all those normally experienced except extremely narrow channels. It is conceivable that some conditions might exist such that the mean-section method would consistently give more satisfactory results than the mid-section method. If the mid-section method is eventually adopted as the "official" method and any field office finds conditions whereby this method does not consistently give satisfactory results, a study should be made by that office to determine the reasons for the inconsistency. The Washington office should be kept informed of and review all studies made in this connection.

The matter of a sufficient number of observations to give satisfactory discharge results is a subject for a study in itself and no attempt was made to do that here. It is noted that, with reasonably good measuring conditions, the usual practice of selecting the number and location of observation stations as used in this study appears satisfactory. This study also shows indirectly that in general more accuracy in discharge measurements can be gained by changing or improving measuring sections and increasing the number of or varying the observations than by improving the method of computation.

The one point these two methods of computation have in common is that the computed areas are the same. This statement oan be proved mathematically, and is shown to be true in aotual practice if the 
figures for partial areas are not rounded. The reason for any difference in discharge is due to weighting the partial areas with different values of velocity. At one time in the past when the mid-section method was proposed to be adopted as the official method, the statement was made that this method was "for computing field notes, and not for taking field notes." This statement is quoted here to emphasize that for practical purposes the use of the mid-section method requires no radical change in the procedure of making discharge measurements, and no revisions in any published or unpublished figures of discharge.

With regard to time savings, the mid-section method of computation consumes less time than the mean-section method. If all the personnel involved in computing and checking discharge measurements were familiar with the mid-section method, it has been shown that there would be a saring of from $\$ 10,000$ to $\$ 12,000$ annually under present day conditions.

In summing up the results found in this comparative study, the following points are brought out:

(1) The mid-section method of computation results in a slightly more nearly accurate figure of discharge, being an average of 0.6 percent closer to the true discharge.

(2) The mid-seotion mothod results in a considerable savings in time as compared to the mean-section method.

(3) There is no appreoiable difference in the field procedure of making a discharge measurement for either method of computation.

Prepared by: Kenneth B. Young

Approved by: F. J. Flynn, Chairman; Hollister Johnson; J. E. MoCall. 